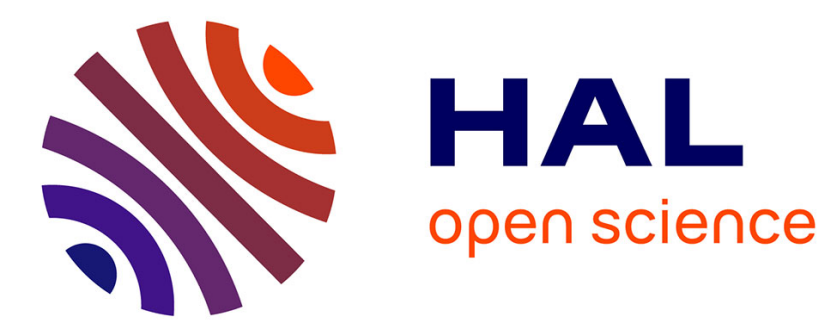

\title{
Normal form theory and nonlinear normal modes: Theoretical settings and applications \\ Cyril Touzé
}

\section{To cite this version:}

Cyril Touzé. Normal form theory and nonlinear normal modes: Theoretical settings and applications. G. Kerschen. Modal Analysis of nonlinear Mechanical Systems,, 555, Springer, 2014, Springer Series CISM courses and lectures, 978-3-7091-1790-2. 10.1007/978-3-7091-1791-0_3 . hal-01134785

\section{HAL Id: hal-01134785 \\ https://hal-ensta-paris.archives-ouvertes.fr/hal-01134785}

Submitted on 24 Mar 2015

HAL is a multi-disciplinary open access archive for the deposit and dissemination of scientific research documents, whether they are published or not. The documents may come from teaching and research institutions in France or abroad, or from public or private research centers.
L'archive ouverte pluridisciplinaire $\mathbf{H A L}$, est destinée au dépôt et à la diffusion de documents scientifiques de niveau recherche, publiés ou non, émanant des établissements d'enseignement et de recherche français ou étrangers, des laboratoires publics ou privés. 


\title{
Normal form theory and nonlinear normal modes: Theoretical settings and applications
}

\author{
Cyril Touzé \\ Unité de Mécanique (UME), \\ ENSTA-ParisTech, \\ Chemin de la Hunière, \\ 91761 Palaiseau Cedex, France. \\ cyril.touzedensta-paristech.fr \\ http://www.ensta.fr/ touze
}

June 26, 2012

\section{Contents}

1 Introduction and notations 2

2 Normal form theory $\quad 3$

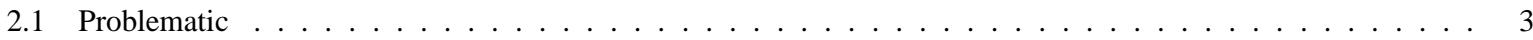

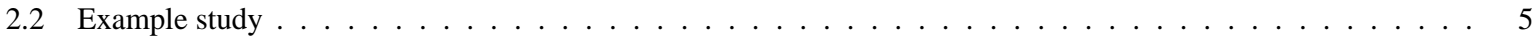

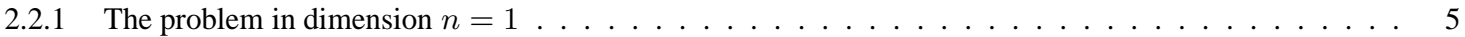

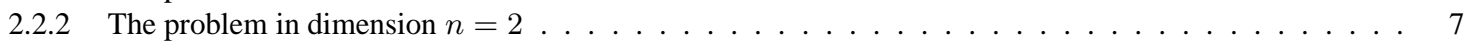

2.3 General case: Poincaré and Poincaré-Dulac theorems . . . . . . . . . . . . . . . . . . . . . . 8

2.4 Application to vibratory systems, undamped case $\ldots \ldots \ldots \ldots \ldots \ldots$

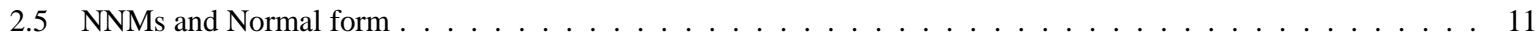

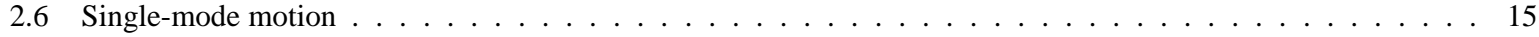

2.7 Classification of nonlinear terms, case of internal resonance . . . . . . . . . . . . . . . . . . . . . 16

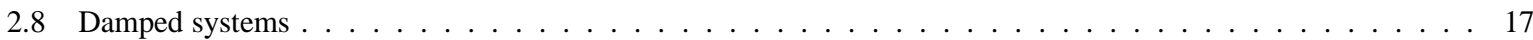

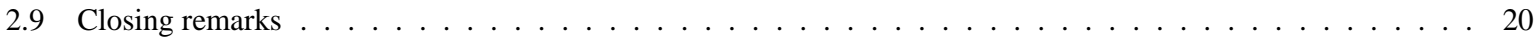

3 Hardening/softening behaviour $\quad 21$

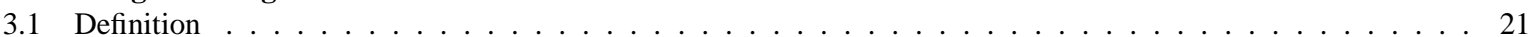

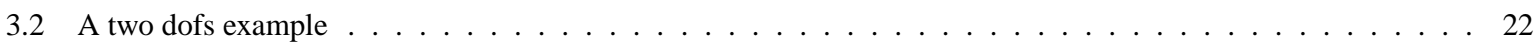

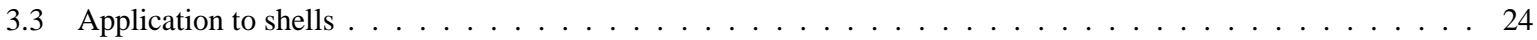

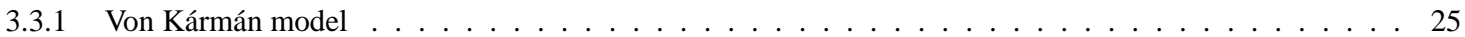

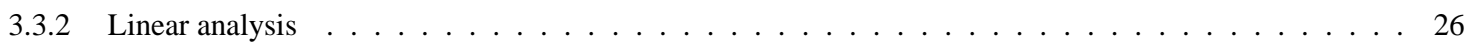

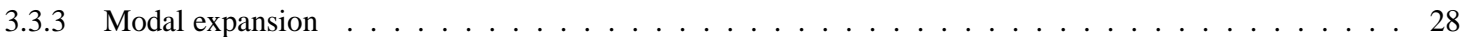

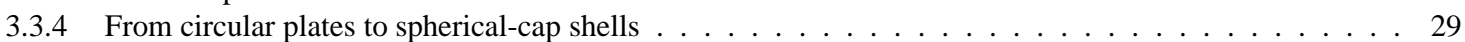

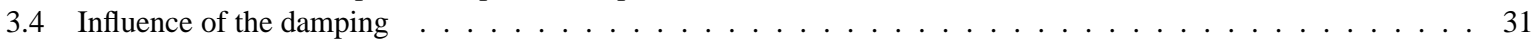

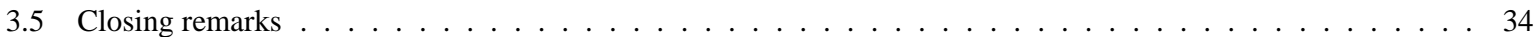

4 Reduced-order models for resonantly forced response $\quad 34$

4.1 Derivation of the reduced-order model f . . . . . . . . . . . . . . . . . . . . . . . . . . 34

4.2 Application : the case of a doubly-curved panel with in-plane inertia . . . . . . . . . . . . . . . . 37

4.3 Application : the case of a closed circular cylindrical shell . . . . . . . . . . . . . . . . . . . 43

4.4 Comparison with the Proper Orthogonal Decomposition method . . . . . . . . . . . . . . . . . . . . . 46

A Expressions of the coefficients for the normal transform in the conservative case 56

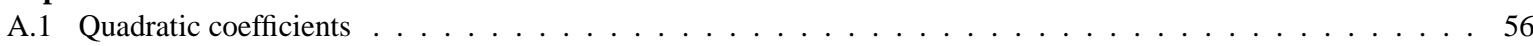

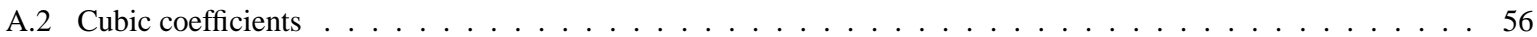




\section{Introduction and notations}

These lecture notes are related to the CISM $^{1}$ course on "Modal Analysis of nonlinear Mechanical systems", held at Udine, Italy, from June 25 to 29, 2012. The key concept at the core of all the lessons given during this week is the notion of Nonlinear Normal Mode (NNM), a theoretical tool allowing one to extend, through some well-chosen assumptions and limitations, the linear modes of vibratory systems, to nonlinear regimes. More precisely concerning these notes, they are intended to show the explicit link between Normal Form theory and NNMs, for the specific case of vibratory systems displaying polynomial type nonlinearities. After a brief introduction reviewing the main concepts for deriving the normal form for a given dynamical system, the relationship between normal form theory and nonlinear normal modes (NNMs) will be the core of the developments. Once the main results presented, application of NNMs to vibration problem where geometric nonlinearity is present, will be highlighted. In particular, the developments of reduced-order models based on NNMs expressed asymptotically with the formalism of real normal form, will be deeply presented. Applications are devoted to thin structures vibrating at large amplitudes, with a special emphasis on thin shells of different geometry (from plates to closed circular cylindrical shells). Effective reduced-order models for the prediction of the type of nonlinearity (hardening/softening behaviour), or the computation of complete bifurcation diagrams for the case of forced vibrations, will be shown, for toy models including a small number of degrees-of-freedom (typicaly two to three dofs), as well as for continuous models such as beams, plates and shells.

The following notations will be used throughout the lecture notes. For generic (nonlinear) dynamical systems, $\mathbf{X}$ will denote the state vector, $\mathbf{X} \in \mathcal{E}$, where $\mathcal{E}$ is the phase space, of dimension $n$. Generally, the simple choice $\mathcal{E} \equiv \mathbb{R}^{n}$ is retained. The dynamical system is denoted as:

$$
\dot{\mathbf{X}}=\mathbf{F}(\mathbf{X}),
$$

with $\mathbf{F}$ the vector field, which could depend on one or more parameters.

For the particular case of vibratory system, geometric nonlinearity is considered, so that only quadratic and cubic type nonlinearities are present in the equations of motions. Hence for nonlinear vibration problems, the generic equations of motion consist in a set of $N$ oscillator equations, denoted under the general formulation:

$$
\forall p=1, \ldots, N: \quad \ddot{X}_{p}+\omega_{p}^{2} X_{p}+\sum_{i=1}^{N} \sum_{j \geq i}^{N} g_{i j}^{p} X_{i} X_{j}+\sum_{i=1}^{N} \sum_{j \geq i}^{N} \sum_{k \geq j}^{N} h_{i j k}^{p} X_{i} X_{j} X_{k}=0,
$$

where $g_{i j}^{p}$ denotes the generic quadratic nonlinear coupling coefficient, and $h_{i j k}^{p}$ the cubic one. In this notation, the upperscript $p$ refers to the oscillator-equation considered, while the subscripts $(i, j, k)$ denotes the coupling monom $X_{i} X_{j} X_{k}$. Note that damping is not considered in Eq. (2). Inclusion of dissipative mechanism will be included in some parts of the notes. Note also that the linear part is diagonal, which means that the variable $X_{p}$ is the modal amplitude of the $\mathrm{p}^{\text {th }}$ linear normal mode. In cases where the linear part is not given as diagonal, a linear change of coordinate can be performed to fit the framework presented here. Finally, to recover the first-order dynamical system formalism, the velocity $Y_{p}=\dot{X}_{p}$ is used as complementary variable, so that, for a collection of $N$ oscillators, the state variable writes: $\mathbf{X}=\left[\begin{array}{lllllll}X_{1} & Y_{1} & X_{2} & Y_{2} & \ldots & X_{N} & Y_{N}\end{array}\right]$, so that $\operatorname{dim}(\mathcal{E})=2 N$.

The first section is devoted to normal form theory. The main idea of introducing a nonlinear transform in order to simplify as much as possible the equations of motion, is first introduced in an illustrative

\footnotetext{
${ }^{1}$ CISM stands for "Centre International des Sciences Mécaniques" (in French), or "International Centre for Mechanical Sciences", see www.cism.it
} 
manner where the reverse problematic is considered. Very simple examples in dimension 1 and 2 allows introducing the key concept of resonance. The core of the theory, the theorems of Poincaré and PoincaréDulac, are then given in a general context, and the result is then specialized to the case of vibratory problems. The main results are given for a conservative problem, and the link to NNMs, is illustrated, allowing derivation of important ideas such as reduced-order modeling and classification of nonlinear terms for physical interpretation. Finally, the case of damped mechanical systems is tackled.

The second section focus on the prediction of the type of nonlinearity (hardening/softening behaviour) for a system of oscillator of the form (2). It is shown that NNMs and normal form gives the correct framework for an efficient and accurate prediction. Examples on two-dofs system, and sphericalcap shells with varying radius of curvature (from the flat plate case to spherical shells), illustrates the method. Finally the influence of the damping on the type of nonlinearity is discussed.

The third section tackles the problem of deriving accurate reduced-order models (ROMs) for thins structures harmonically excited at resonance, in the vicinity of one of its eigenfrequency, and vibrating at large amplitudes. NNMs and normal form are used to derive the ROMs, and examples on different shells are shown: a doubly-curved (hyperbolic paraboloid) panel illustrates a case without internal resonance, while a closed circular cylindrical shell allows illustrating a more complicated case with a 1:1 internal resonance. the complete bifurcation diagram with different kind of bifurcation points are clearly recovered by the ROM, and a comparison with the most routinely used Proper Orthogonal Decomposition method (POD) is shown to conclude the notes.

\section{Normal form theory}

Normal form theory is a classical tool in the analysis of dynamical systems, and general introductions can be found in many textbooks, see e.g. [20, 21, 57, 29]. It is generally used in bifurcation theory in order to define the simplest form of dynamical systems generating classical bifurcations of increasing co-dimension. Here however, the general theory will be used for another purpose: defining a nonlinear change of coordinates allowing one to express the dynamics in an invariant-based span of the phase space, where the (curved) generating axis are the invariant manifolds arising from the linear eigenspaces, i.e. the NNMs of the system.

Normal form theory is based on two major theorems, due to Poincaré and Poincaré-Dulac, which have been demonstrated in the beginning of the $\mathrm{XX}^{\text {th }}$ century $[39,13]$. The main idea is to simplify, as far as possible, the equations of motion of a nonlinear dynamical systems, by means of nonlinear change of coordinates. The presentation will begin with a reverse problematic, in order to understand better the main issue: starting from a simple, linear problem, we will show how a nonlinear change of coordinate can make it appear as complicated. Then the normal form will be introduced gradually with two examples study, starting from the simplest cases with dimension 1 and 2. The "reverse" illustrative problematic is borrowed from [30], and the presentation of normal form used follows closely that shown in $[30,29]$.

\subsection{Problematic}

Before entering the complicated calculations for tackling the general case, let us first introduce an illustrative example, allowing us to properly define the goal pursued, which is defined as trying to simplify as far as possible, in the vicinity of a particular solution (e.g. fixed point or periodic orbit), a given dynamical system.

To begin with, let us consider the simple initial-value problem:

$$
\begin{aligned}
& \frac{\mathrm{d}^{2} Y}{\mathrm{~d} t^{2}}+Y=0, \\
& Y(t=0)=Y_{0}, \quad \frac{\mathrm{d} Y}{\mathrm{~d} t}(t=0)=0,
\end{aligned}
$$


where $Y$ is a real coordinate depending on time $t$. This equation is that of a single oscillator, with eigenfrequency equal to one. Its solution in time is known and reads:

$$
Y(t)=Y_{0} \cos t .
$$

Now let us introduce the transformed variable:

$$
X=\exp (Y)-1 .
$$

Differentiating Eq. (5) two times with respect to time $t$, and inserting in the original dynamical equation (3a), one can show that the transformed variable $X$ satisfy the following evolution equation:

$$
(1+X) \ddot{X}-\dot{X}^{2}+(1+X)^{2} \ln (1+X)=0,
$$

where $\ln$ is the natural logarithm. In the case where one would have to face a physical problem expressed by Eq. (6), with appropriate initial conditions, then the solution would have been more difficult to find ! Maybe that with a great intuition and a bit of luck, one could have find the solution which, in our case, is known by construction, and is simply given by:

$$
X(t)=\exp \left(Y_{0} \cos t\right)-1 .
$$
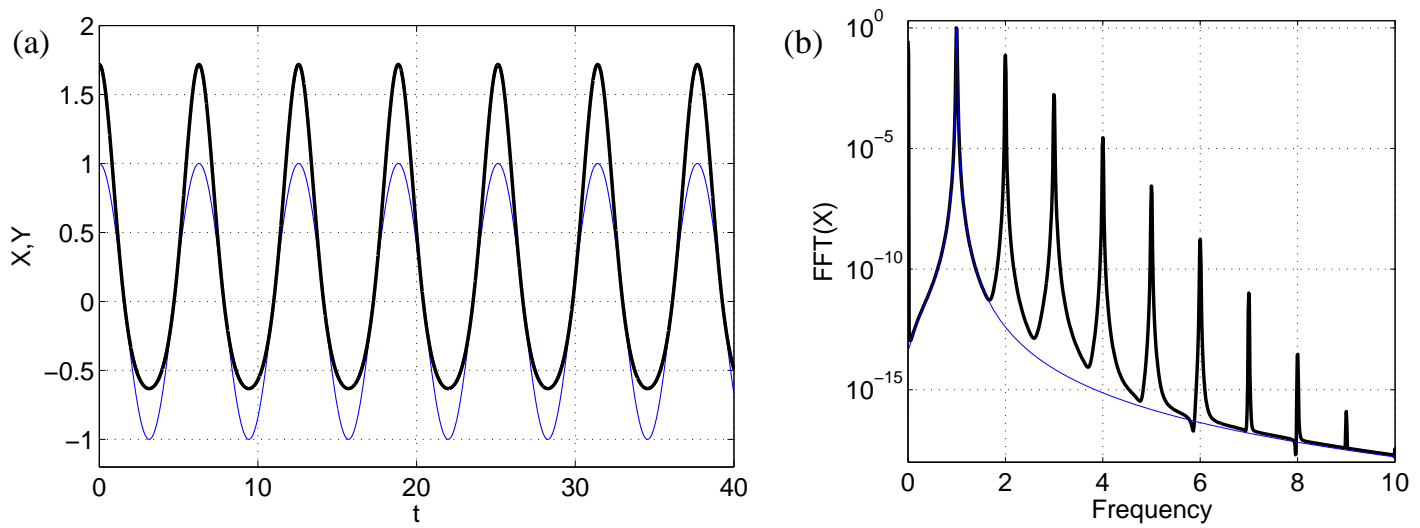

Figure 1: (a) Time series of the transformed variable $X$ (black solid line), compared to the original $Y$ (thin blue line), for the problem defined through Eqs (3)-(7). (b): Fourier Transform of $X$ (black solid line) and $Y$ (thin blue line), showing their harmonic content.

The solution for $X$ is represented in Fig. 1. As awaited, it is $2 \pi$-periodic, but contrary to the initial solution for $Y$ displaying only one harmonic component, the $X$ solution shows an infinity of harmonics, with exponential decay in amplitude, as revealed by the Fourier transform of $X$, Fig. 1(b). This example shows that the apparent complexity of the problem for $X$ only results from a nonlinear change of coordinates. Looking at the things in a reverse manner, the question arising is naturally: for a given problem, is it possible to find such a nonlinear transformation that could simplify, at best linearise, the initial system ? The main idea of normal form theory is to give an answer to this question. Without inspired intuition, one could at least try an asymptotic, power series expansion, in order to get an idea of the sought nonlinear change of coordinates. This functional relationship has to be defined in the vicinity of $Y=0$ (or equivalently, $X=0$ ), which is the fixed point of the original dynamical system (equivalently, of the transformed system). Once again the result is known and reads, in our case:

$$
X=\sum_{n=1}^{+\infty} \frac{1}{n !} Y^{n} .
$$


Note that $X \sim Y$ for $X, Y$ small, which means that the transformation is identity-tangent, and aims at conserving the linear characteristic of the original system. In a general case where the solution is not known, one would like to find iteratively, order by order, the nonlinear transformation.

Let us close these introductory remarks by precising what is meant by "as far as possible", in the definition of the nonlinear transform. Consider the general dynamical system $\dot{X}=F(X)$, where $F(X)=X+X^{p}$, in the vicintiy of the fixed point $X=0$. The common sense would state that this problem with $p=6$ is "more nonlinear" than the same with $p=2$. However, as shown in Fig. 2, the linear approximation $F(X) \sim X$ is valid on a larger range of $X$-amplitudes for $p=6$ than for $p=2$. Hence the dynamical system with $F(X)=X+X^{6}$ is "less nonlinear" than the one with $F(X)=X+X^{2}$, because the nonlinearities are activated for larger values of the amplitude $X$, and one could give confidence to a linear approximation on a larger range of amplitudes.

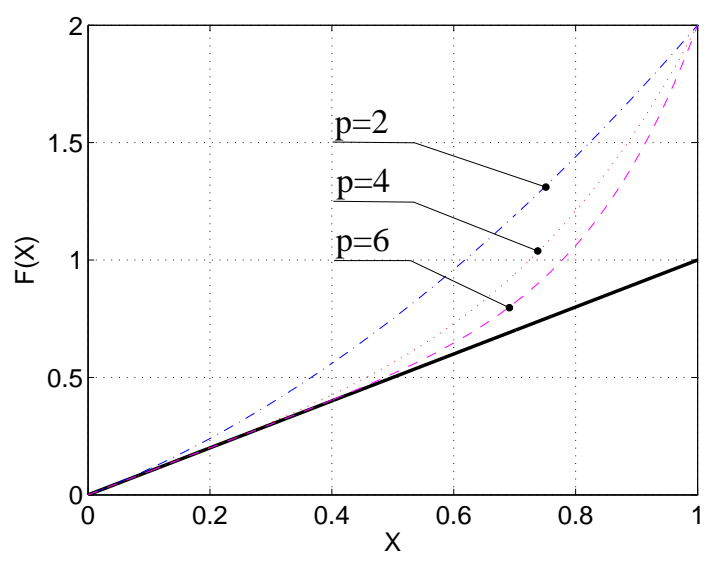

Figure 2: Representation of $F(X)=X+X^{p}$ for $p=2,4,6$, as compared to the linear case.

Finally, the goal can now be stated properly: for a given dynamical system, in the vicinity of a particular solution (typically a fixed point), we would like to find out nonlinear transformations that aims at extending the validity range of the linear approximation, by cancelling a maximum number of monoms of increasing orders, in the power series expansion of the vector field. The next subsection introduces the normal form transform for simple problems where the phase space have dimension 1 and 2, before generalizing the results for any dimension and any dynamical system.

\subsection{Example study}

\subsubsection{The problem in dimension $n=1$}

Let us consider a dynamical system:

$$
\dot{X}=s X+a_{2} X^{2}+a_{3} X^{3}+\ldots=s X+\sum_{p>1} a_{p} X^{p},
$$

where $X \in \mathbb{R}$ (phase space of dimension $n=1$ ). $X=0$ is a hyperbolic fixed point as long as $s \neq 0$, otherwise a marginal case is at hand (bifurcation point).

Let us introduce a nonlinear transform:

$$
X=Y+\alpha_{2} Y^{2}
$$

where $\alpha_{2}$ is introduced in order to cancel the quadratic monom of the original system, i.e. $a_{2} X^{2}$ in Eq. (9). Here $Y$ is the new variable, and the goal of the transformation is to obtain a dynamical system for 
the new unknown $Y$ that is simpler than the original one. Differentiating (10) with respect to time and substituting in (9) gives:

$$
\left(1+2 \alpha_{2} Y\right) \dot{Y}=s Y+\left(s \alpha_{2}+a_{2}\right) Y^{2}+\mathcal{O}\left(Y^{3}\right)
$$

All the calculation are realized in the vicinity of the fixed point. Hence $Y \ll 1$, so that one can mutliply both sides by:

$$
\left(1+2 \alpha_{2} Y\right)^{-1}=\sum_{p=0}^{+\infty}(-2)^{p} \alpha_{2}^{p} Y^{p}
$$

Rearranging the terms by increasing orders finally leads to:

$$
\dot{Y}=s Y+\left(a_{2}-s \alpha_{2}\right) Y^{2}+\mathcal{O}\left(Y^{3}\right)
$$

From that equation, it appears clearly that the quadratic term can be cancelled by selecting:

$$
\alpha_{2}=\frac{a_{2}}{s},
$$

which is possible as long as $s \neq 0$. This condition has been assumed at the beginning (hyperbolicity of the fixed point). Hence with that choice, the objective is fulfilled, the nonlinearity has been repelled to order three. The only condition appearing is that of hyperbolicity, which means that we are not at a (singular) bifurcation point. The process can be continued further, let us examine what happens for the third order. First, one has to write the resulting dynamical system for $Y$, by expliciting the thirdorder term, which has been modified after the first transform that has cancelled the quadratic monom. Substituting for (10) in (9), with the retained choice $\alpha_{2}=a_{2} / s$, leads to:

$$
\dot{Y}=s Y+\bar{a}_{3} Y^{3}+\mathcal{O}\left(Y^{4}\right), \quad \text { where } \quad \bar{a}_{3}=a_{3}+2 a_{2}^{2} / s
$$

A cubic transform is now introduced in order to simplify (15):

$$
Y=Z+\alpha_{3} Z^{3},
$$

Repeating the same procedure leads to:

$$
\dot{Z}=s Z+\left(\bar{a}_{3}-2 s \alpha_{3}\right) Y^{3}+\mathcal{O}\left(Y^{4}\right),
$$

which shows that the cubic term can be cancelled by selecting:

$$
\alpha_{3}=\frac{\bar{a}_{3}}{2 s} \text {. }
$$

Once again, the only condition appearing in the calculation is the one already found, $s \neq 0$. The complete change of coordinate can be written up to third order by gathering together Eqs. (10) and (16):

$$
X=Z+\frac{a_{2}}{s} Z^{2}+\frac{\bar{a}_{3}}{2 s} Z^{3}+\mathcal{O}\left(Z^{4}\right),
$$

and the resulting dynamical system for the knew unknown $Z$ now reads:

$$
\dot{Z}=s Z+\bar{a}_{4} Z^{4}+\mathcal{O}\left(Z^{5}\right),
$$

where $\bar{a}_{4}$ has to be computed properly by replacing (10) in (9) and carefully selecting fourth-order terms. This process is more and more difficult with increasing orders, and rapidly suggest for helping oneself with symbolic computation softwares. In any case we can conclude that:

- The objective is fulfilled: nonlinearities have been repelled up to fourth order. The process can be continued until complete linearisation of the problem, which is possible as long as $s \neq 0$ (hyperbolicity condition).

- The computed nonlinear transform becomes singular when one aproaches the marginality for $s \longrightarrow 0$, because the $\alpha_{i}$ 's scale as $1 / s$. This is a reflection of the fact that the nonlinearities dominates the dynamical behaviour in the vicinity of the bifurcation point; however the linearisation is still possible as long as one is away from that point. 


\subsubsection{The problem in dimension $n=2$}

Before generalizing the result, the problem in a phase space of dimension 2 is now tackled. In dimensions $n \geq 2$, resonance conditions appear, hence making the problem a little bit more complicated than what could be expected from the precedent subsection, where the hyperbolicity condition has been found to be sufficient for simplifying the original system.

We consider $\mathbf{X}=\left(\begin{array}{ll}X_{1} & X_{2}\end{array}\right)^{t} \in \mathbb{R}^{2}$, and for simplicity the dynamical system $\dot{\mathbf{X}}=F(\mathbf{X})$ is considered quadratic in $\mathbf{X}$. We also assume that the linear part is diagonal and that the two eigenvalues reads $\left(s_{1}, s_{2}\right)$. The system can be written explicitely as:

$$
\begin{aligned}
& \dot{X}_{1}=s_{1} X_{1}+a_{11}^{1} X_{1}^{2}+a_{12}^{1} X_{1} X_{2}+a_{22}^{1} X_{2}^{2}, \\
& \dot{X}_{2}=s_{2} X_{2}+a_{11}^{2} X_{1}^{2}+a_{12}^{2} X_{1} X_{2}+a_{22}^{2} X_{2}^{2} .
\end{aligned}
$$

The nonlinear transform is introduced:

$$
\begin{aligned}
& X_{1}=Y_{1}+\alpha_{11}^{1} Y_{1}^{2}+\alpha_{12}^{1} Y_{1} Y_{2}+\alpha_{22}^{1} Y_{2}^{2}, \\
& X_{2}=Y_{2}+\alpha_{11}^{2} Y_{1}^{2}+\alpha_{12}^{2} Y_{1} Y_{2}+\alpha_{22}^{2} Y_{2}^{2} .
\end{aligned}
$$

Differentiating (22) with respect to time and replacing in (21) leads to:

$$
\begin{aligned}
& \left(1+2 \alpha_{11}^{1} Y_{1}+\alpha_{12}^{1} Y_{2}\right) \dot{Y}_{1}+\left(\alpha_{12}^{1} Y_{1}+2 \alpha_{22}^{1} Y_{2}\right) \dot{Y}_{2} \\
& \quad=s_{1} Y_{1}+\left(a_{11}^{1}+s_{1} \alpha_{11}^{1}\right) Y_{1}^{2}+\left(a_{12}^{1}+s_{1} \alpha_{12}^{1}\right) Y_{1} Y_{2}+\left(a_{22}^{1}+s_{1} \alpha_{22}^{1}\right) Y_{2}^{2}+\mathcal{O}\left(Y_{1,2}^{3}\right) \\
& \left(1+2 \alpha_{22}^{2} Y_{2}+\alpha_{12}^{2} Y_{1}\right) \dot{Y}_{2}+\left(\alpha_{12}^{2} Y_{2}+2 \alpha_{11}^{2} Y_{1}\right) \dot{Y}_{1} \\
& \quad=s_{2} Y_{2}+\left(a_{11}^{2}+s_{2} \alpha_{11}^{2}\right) Y_{1}^{2}+\left(a_{12}^{2}+s_{2} \alpha_{12}^{2}\right) Y_{1} Y_{2}+\left(a_{22}^{2}+s_{2} \alpha_{22}^{2}\right) Y_{2}^{2}+\mathcal{O}\left(Y_{1,2}^{3}\right)
\end{aligned}
$$

The calculation is pursued by noting that, at the lowest order, one has:

$$
\begin{aligned}
& \dot{Y}_{1}=s_{1} Y_{1}+\mathcal{O}\left(Y_{1,2}^{2}\right), \\
& \dot{Y}_{2}=s_{2} Y_{2}+\mathcal{O}\left(Y_{1,2}^{2}\right),
\end{aligned}
$$

Hence the derivatives with respect to time involving a product in (23) can be replaced thanks to $Y_{i} \dot{Y}_{j}=$ $s_{j} Y_{i} Y_{j}+\mathcal{O}\left(Y_{1,2}^{3}\right)$, so that finally one obtains:

$$
\begin{aligned}
\dot{Y}_{1}=s_{1} Y_{1}+ & \left(a_{11}^{1}-s_{1} \alpha_{11}^{1}\right) Y_{1}^{2}+\left(a_{12}^{1}-s_{2} \alpha_{12}^{1}\right) Y_{1} Y_{2} \\
& +\left(a_{22}^{1}+\left(s_{1}-2 s_{2}\right) \alpha_{22}^{1}\right) Y_{2}^{2} \\
\dot{Y}_{2}=s_{2} Y_{2}+ & \left(a_{11}^{2}+\left(s_{2}-2 s_{1}\right) \alpha_{11}^{2}\right) Y_{1}^{2}+\left(a_{12}^{2}-s_{1} \alpha_{12}^{2}\right) Y_{1} Y_{2} \\
+ & \left(a_{22}^{2}-s_{2} \alpha_{22}^{2}\right) Y_{2}^{2}
\end{aligned}
$$

In the previous equations, the unkonwns $\left\{\alpha_{i j}^{p}\right\}$ can be found by setting:

$$
\begin{aligned}
& \alpha_{11}^{1}=\frac{a_{11}^{1}}{s_{1}}, \quad \alpha_{12}^{1}=\frac{a_{12}^{1}}{s_{2}}, \quad \alpha_{22}^{1}=\frac{a_{22}^{1}}{2 s_{2}-s_{1}}, \\
& \alpha_{11}^{2}=\frac{a_{11}^{2}}{2 s_{1}-s_{2}}, \quad \alpha_{12}^{2}=\frac{a_{12}^{2}}{s_{1}}, \quad \alpha_{22}^{2}=\frac{a_{22}^{2}}{s_{2}},
\end{aligned}
$$

so that the complete cancellation of all the nonlinear terms present in the original equations (21) is possible if and only if:

$$
s_{1} \neq 0, \quad s_{2} \neq 0, \quad s_{2} \neq 2 s_{1}, \quad s_{1} \neq 2 s_{2} .
$$

The first two conditions have already been encoutered in the precedent subsection in dimension $n=1$ : once again, they are the consequence of the assumption of hyperbolicity of the fixed point, stating that 
we are not at a (marginal) bifurcation point. The last two conditions are new and result from extending the dimension to $n=2$. They are called in the remainder resonance condition, and they reflect the fact that the eigenvalues may share a commensurability relationship. Hence they are completely different from the hyperbolicity condition. When such a relationship exists between the eigenvalues, e.g. when $s_{2}=2 s_{1}$, the analysis shows that the system can not be linearized. More precisely, a monom, present in the original equations, can not be cancelled because it is resonant through the eigenvalue relationship, and thus strongly couple the two equations. In the analysis, the resulting normal form keeps the monom and the dynamics is different from a linearizable case. For example, in the case $s_{2}=2 s_{1}$ (assuming $s_{1}, s_{2} \neq 0$ ), the system can be simplified at best to:

$$
\begin{aligned}
& \dot{Y}_{1}=s_{1} Y_{1}, \\
& \dot{Y}_{2}=s_{2} Y_{2}+a_{11}^{2} Y_{1}^{2} .
\end{aligned}
$$

The resonance condition can be understood in the following manner: from the first equation we have $Y_{1}(t) \propto \exp s_{1} t$. Reporting in the second equation, the nonlinear term makes appear a term proportional to $\exp 2 s_{1} t=\exp s_{2} t$. Consequently the nonlinear term in (29b) may be interpreted as a forcing term, acting precisely at the resonance eigenvalue $s_{2}$ of the second equation. The solutions of this nonhomogeneous differential equation lead to secular terms, which explains why the monom can not be cancelled. These resonance conditions are further analyzed in subsection 2.4 , devoted to vibratory systems.

This subsection reveals the core of Poincaré and Poincaré-Dulac theorems. When no resonance condition exist between the eigenvalues, then the system is equivalent to a linear one (Poincaré). In case of resonance relationships, the system can be simplified thanks to successive nonlinear transforms. Only the resonant monoms stay in the resulting so-called normal form of the problem (Poincaré-Dulac).

\subsection{General case: Poincaré and Poincaré-Dulac theorems}

We are now in position to extend the result to a general case in dimension $n$. Let us denote the dynamical system as:

$$
\dot{\mathbf{X}}=\mathbf{F}(\mathbf{X})=\mathbf{L X}+\mathbf{N}_{2}(\mathbf{X})+\ldots+\mathbf{N}_{p}(\mathbf{X})
$$

where $\mathbf{X} \in \mathcal{E}$ ( $\mathcal{E} \equiv \mathbb{R}^{n}$ otherwise stated), $\mathbf{L}$, the linear part, is assumed to be diagonal ${ }^{2}$, with $\mathbf{L} \equiv$ $\operatorname{diag}\left(s_{p}\right), \mathbf{N}_{p}$ denotes the terms at order $p$.

The generalization of the resonance condition reads, for a given order of nonlinearity $p \geq 2$ :

$$
s_{j}=\sum_{i} n_{i} s_{i}, \quad n_{i} \geq 0, \quad \sum_{i} n_{i}=p .
$$

Note that the cancellation of non-resonant monoms via nonlinear transformations, is realized sequentially, order by order, so that the resonance condition appears by increasing orders $p$. For $p=2$, Eq. (31) make appear the conditions $s_{j}=2 s_{i}$ encountered on the precedent example, as well as a case involving three eigenvalues: $s_{i}=s_{j}+s_{k}$, that can be found only when the dimension is such that $n \geq 3$.

When no resonance condition of the form (31) exist between the eigenvalues of the system, Poincaré theorem states that a nonlinear transform, $\mathbf{X}=\mathbf{Y}+\mathbf{g}(\mathbf{Y})$ exist, such that the dynamics for the new coordinate $\mathbf{Y}$ is linear and simply writes: $\dot{\mathbf{Y}}=\mathbf{L Y}$. This theorem has been extended by Dulac in 1917 to the case of the existence of resonance condition, stating (Poincaré-Dulac theorem) that a nonlinear transform $\mathbf{X}=\mathbf{Y}+\mathbf{g}(\mathbf{Y})$ exist such that the resulting system for the new variable $\mathbf{Y}$ is still nonlinear but drastically simplified as compared to the original, as it contains only the resonant monoms that can

\footnotetext{
${ }^{2}$ The particular cases where the linear operator is not diagonalisable are not treated here for the sake of brevity. The normal form theory applies in these cases as well, one has just to use the Jordan representation of the linear operator to obtain generic cases, some examples can be found in classical textbooks, see e.g. [21, 30, 57].
} 
not be cancelled. In both cases, the simplified system $\dot{\mathbf{Y}}=\mathbf{f}(\mathbf{Y})$ is called the normal form of the original system. In the simplest case the normal form is a linear system, in the other cases it contains only the resonant monoms, resulting from the resonance conditions between the eigenvalues.

These theorems are very strong in the sense that they underline the fact that, in the vicinity of a particular solution, the eigenspectrum completely determines the nonlinear part. This means in particular that, from the knowledge of the eigenvalues, one is able to construct the normal form of the system by deriving the resonant monoms. One must keep however in mind that the theory is local only, and is valid only in the vicinity of a particular solution. Finally, the method for demonstrating the theorems is a sequential calculations that treats by successive orders of nonlinearity the monoms $\mathbf{N}_{p}$ appearing in the dynamical system (30). This sequential calculation can be automatized by using symbolic toolbox softwares, nonetheless it becomes more and mode difficult with increasing orders. And it shows that the method do not lend itself to a numerical treatment if one would like to find numerically the nonlinear change of coordinates.

\subsection{Application to vibratory systems, undamped case}

The case of vibratory systems displaying quadratic and cubic nonlinearities, is now examined, following the general notation stated in the introduction, Eq. (2). At present damping is not considered, in order to introduce properly the concepts and the Nonlinear normal modes. The results are extended to dissipative vibratory systems in subsection 2.8. In this subsection, the presentation follows closely the theoretical results published in [55].

The eigenvalues of a vibratory system are complex conjugate, $\pm i \omega_{p}, p=1, \ldots \mathrm{N}$. To compute the normal form of Eq. (2), a first idea would be to follow strictly the general framework sketched in the precedent subsection. This would mean to express Eq. (2) as:

$$
\dot{\mathbf{X}}=\mathbf{F}(\mathbf{X})=\mathbf{L X}+\mathbf{N}_{2}(\mathbf{X})+\mathbf{N}_{3}(\mathbf{X}),
$$

where the linear part $\mathbf{L}$ is diagonal, and thus reads: $\mathbf{L}=\operatorname{diag}\left\{ \pm i \omega_{p}\right\}$. For example, this framework has been first used by Jézéquel and Lamarque [22] to express how the nonlinear normal modes can be introduced from the framework provided by normal form theory. However a different choice is retained here. The linear part will be retained as it is when the system is written at first-order by using the velocity $Y_{p}=\dot{X}_{p}$ as auxiliary variable, i.e. a block-diagonal matrix, each block being written as:

$$
\left(\begin{array}{cc}
0 & 1 \\
-\omega_{p}^{2} & 0
\end{array}\right)
$$

This choice is done in order to avoid introducing complex quantities in the calculations. It allows also to have equations that will be of oscillator form at each stage of the calculation, so that the auxiliary variable can always be cancelled in order to recover oscillator-like equations beginning with $\ddot{X}+X+\ldots$. This so-called real formulation allows simpler physical interpretation of the different terms, as it will be shown next.

A second point of interest is a consequence of the purely imaginary eigenspectrum. With regard to the resonance relationships highlighted in the previous subsection, it appears that a complete linearization is not possible in any case for a conservative vibratory system. Indeed, if only one oscillator equation is considered, then one can always fulfill the following relationship between the two complex conjugate eigenvalues $\{+i \omega,-i \omega\}$ :

$$
+i \omega=+i \omega+i \omega-i \omega
$$

i.e. a relationship of the form (31) with order $p=3$. Coming back to the oscillator equation, this means that the Duffing equation:

$$
\ddot{X}+\omega^{2} X+\alpha X^{3}=0,
$$


is under its normal form, the cubic term (monom associated with the resonance relation (34)) can not be cancelled through a nonlinear transform. On a physical viewpoint, this stands as a good news. Indeed, one of the most important observed feature in nonlinear oscillations is the frequency dependence upon vibration amplitude. If the system could be linearized, this would mean that the underlying dynamics is linear, hence the frequency should not change with the amplitude. Note that this frequency dependence on amplitude is the main topic of section 3 , and thus is deeply investigated there.

In the general case where $N$ oscillator-equations are considered, numerous resonance relationships of the form:

$$
+i \omega_{p}=+i \omega_{p}+i \omega_{k}-i \omega_{k}
$$

are possible, for arbitrary $p, k \in[1, N]^{2}$. This means that the original system (2) can be simplified, but numerous terms will remain at the end of the process, in the normal form, following Poincaré-Dulac theorem. However, as it will be shown next, the game is worth the candle, as numerous important terms will be cancelled, and also because the remaining terms can be easily interpreted.

Before stating the main result, a last point must be underlined. The resonance relationships put forward through Eqs (34)-(36) are denoted as trivial: they exist whatever the values of the frequencies of the studied structure are. A second family of resonance relationships may arise from internal resonances between the eigenfrequencies of the system. For example, order-two internal resonances reads, for arbitrary $(p, i, j)$ :

$$
\omega_{p}=\omega_{i}+\omega_{j}, \quad \omega_{p}=2 \omega_{i}
$$

while third-order (linked to cubic nonlinear coupling terms) writes, for arbitrary $(p, i, j, k)$ :

$$
\omega_{p}=\omega_{i}+\omega_{j} \pm \omega_{k}, \quad \omega_{p}=2 \omega_{i} \pm \omega_{j}, \quad \omega_{p}=3 \omega_{i} .
$$

These internal resonances may exist, or not, depending on the eigenfrequencies of the structure. In the remainder of the lecture, a clear distinction is thus made between trivial resonance relationships and internal resonance. The main result is given for the case where no internal resonance exist in the system. The case of their presence is not problematic and is handled in subsection 2.5.

Let us give the general result. Considering a vibrating system of $N$ oscillator-modes, written as a first-order dynamical system by keeping oscillator-blocks in the linear part (real formulation), and assuming no internal resonance between the eigenfrequencies $\left\{\omega_{p}\right\}_{p=1 \ldots N}$, a nonlinear transform can be found in order to cancel the maximum number of quadratic and cubic coupling terms present in the original system. The nonlinear transform reads, up to order three:

$$
\begin{gathered}
X_{p}=R_{p}+\sum_{i=1}^{N} \sum_{j \geq i}^{N}\left(a_{i j}^{p} R_{i} R_{j}+b_{i j}^{p} S_{i} S_{j}\right) \\
+\sum_{i=1}^{N} \sum_{j \geq i}^{N} \sum_{k \geq j}^{N} r_{i j k}^{p} R_{i} R_{j} R_{k}+\sum_{i=1}^{N} \sum_{j=1}^{N} \sum_{k \geq j}^{N} u_{i j k}^{p} R_{i} S_{j} S_{k}, \\
Y_{p}=S_{p}+\sum_{i=1}^{N} \sum_{j=1}^{N} \gamma_{i j}^{p} R_{i} S_{j}+\sum_{i=1}^{N} \sum_{j \geq i}^{N} \sum_{k \geq j}^{N} \mu_{i j k}^{p} S_{i} S_{j} S_{k}+\sum_{i=1}^{N} \sum_{j=1}^{N} \sum_{k \geq j}^{N} \nu_{i j k}^{p} S_{i} R_{j} R_{k} .
\end{gathered}
$$

In these equations, the new-defined variables, $R_{p}$ and $S_{p}$, are respectively homogeneous to a displacement and a velocity. They are called the normal coordinates. The coefficients of this non-linear change of variables $\left(a_{i j}^{p}, b_{i j}^{p}, r_{i j k}^{p}, u_{i j k}^{p}, \gamma_{i j}^{p}, \mu_{i j k}^{p}, \nu_{i j k}^{p}\right)$ are given in Appendix A, as well as in [55]. the complete proof leading to Eqs. (39) is given in [49]. 
The normal form is found by substituting for Eq. (39) in (2), and reads:

$$
\begin{aligned}
\forall p= & 1, \ldots, N: \\
\dot{R}_{p}= & S_{p}, \\
\dot{S}_{p}= & -\omega_{p}^{2} R_{p}-\left(A_{p p p}^{p}+h_{p p p}^{p}\right) R_{p}^{3}-B_{p p p}^{p} R_{p} S_{p}^{2} \\
& -R_{p}\left[\sum_{j>p}^{N}\left[\left(A_{j p j}^{p}+A_{p j j}^{p}+h_{p j j}^{p}\right) R_{j}^{2}+B_{p j j}^{p} S_{j}^{2}\right]+\sum_{i<p}\left[\left(A_{i i p}^{p}+A_{p i i}^{p}+h_{i i p}^{p}\right) R_{i}^{2}+B_{p i i}^{p} S_{i}^{2}\right]\right] \\
& -S_{p}\left[\sum_{j>p}^{N} B_{j p j}^{p} R_{j} S_{j}+\sum_{i<p} B_{i i p}^{p} R_{i} S_{i}\right] .
\end{aligned}
$$

In these last equations, new coeffients $\left(A_{i j k}^{p}, B_{i j k}^{p}\right)$ appear: they arise from the cancellation of the quadratic terms, and write:

$$
\begin{aligned}
& A_{i j k}^{p}=\sum_{l \geq i}^{N} g_{i l}^{p} a_{j k}^{l}+\sum_{l \leq i} g_{l i}^{p} a_{j k}^{l}, \\
& B_{i j k}^{p}=\sum_{l \geq i}^{N} g_{i l}^{p} b_{j k}^{l}+\sum_{l \leq i} g_{l i}^{p} b_{j k}^{l} .
\end{aligned}
$$

Equations (39)-(40) constitutes the main result for vibratory systems. The following comments are worth mentionable:

- Even though the last Eq. (40) appears longer on the page than the original one (2), the reader must be convinced that it is simpler. In particular, one can observe that a nonlinear term of the form $R_{i}^{2}$ or $R_{i}^{3}$ does not exist anymore in the equation for the $\mathrm{p}^{\text {th }}$ normal coordinate $R_{p}$. These monoms are particularly important and are called invariant-breaking terms. Their cancellation will be related to nonlinear normal modes and invariant manifold in the next subsection.

- Thanks to the real formulation, oscillator-equations are obtained for the normal form. One can easily recover second-order oscillator equations by substituting for (40a) in (40b). This formalism allows for simple physical meaning of the different terms.

- Eq. (39) is identity-tangent: the linear results are thus recovered for small amplitudes.

- Velocity-dependent terms arise in Eqs. (40) only if quadratic non-linearity is present in the initial problem given by (2). For example, for a non-linear beam problem, for which $g_{i j}^{p} \equiv 0$, no velocitydependent terms appear when considering the third-order approximation of the dynamics onto invariant manifolds.

\subsection{NNMs and Normal form}

Nonlinear Normal Modes (NNMs) is the core of this CISM course on Modal analysis of nonlinear systems. The main idea of defining a NNM is to try extend to notion of linear normal modes at the nonlinear stage. Linear modes offer a very interesting basis for analyzing and understanding the dynamics of linear systems. Their main property relies in the decoupling of the equations of motion, resulting in superposition theorem. This decoupling can be interpreted, on a geometrical viewpoint, in the phase space of the system, as an invariance property of the linear eigenspaces, which are two-dimensional invariant planes of the linear system.

The idea of defining a non-linear normal mode is to extend the decoupling of the linear eigenspaces exhibited at the linear stage. Letting $g_{i j}^{p} \equiv h_{i j k}^{p} \equiv 0$ in Eq. (2), and initiating a motion along the $p^{t h}$ 


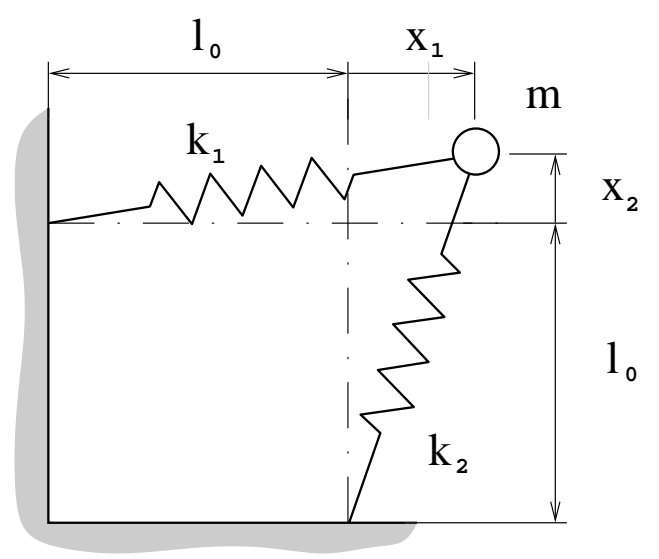

Figure 3: Schematics of the system containing quadratic and cubic nonlinearities

eigendirection results in a motion which is always contained within it. This is the invariance property one would be able to extend to the non-linear regime.

Let us introduce a simple two-dofs example in order to illustrate the problem. The system, composed of a mass connected to two nonlinear springs, is represented in figure 3. The dynamics of the system is described by :

$$
\begin{aligned}
& \ddot{X}_{1}+\omega_{1}^{2} X_{1}+\frac{\omega_{1}^{2}}{2}\left(3 X_{1}^{2}+X_{2}^{2}\right)+\omega_{2}^{2} X_{1} X_{2}+\frac{\omega_{1}^{2}+\omega_{2}^{2}}{2} X_{1}\left(X_{1}^{2}+X_{2}^{2}\right)=0, \\
& \ddot{X}_{2}+\omega_{2}^{2} X_{2}+\frac{\omega_{2}^{2}}{2}\left(3 X_{2}^{2}+X_{1}^{2}\right)+\omega_{1}^{2} X_{1} X_{2}+\frac{\omega_{1}^{2}+\omega_{2}^{2}}{2} X_{2}\left(X_{1}^{2}+X_{2}^{2}\right)=0,
\end{aligned}
$$

where $X_{1}=x_{1} / l_{0}$ and $X_{2}=x_{2} / l_{0}$ have been nondimensionalized by the length $l_{0}$ of the springs at rest. The system is fully parameterized by the two eigenfrequencies $\left(\omega_{1}, \omega_{2}\right)$. The phase space is $\mathbb{R}^{4}$,

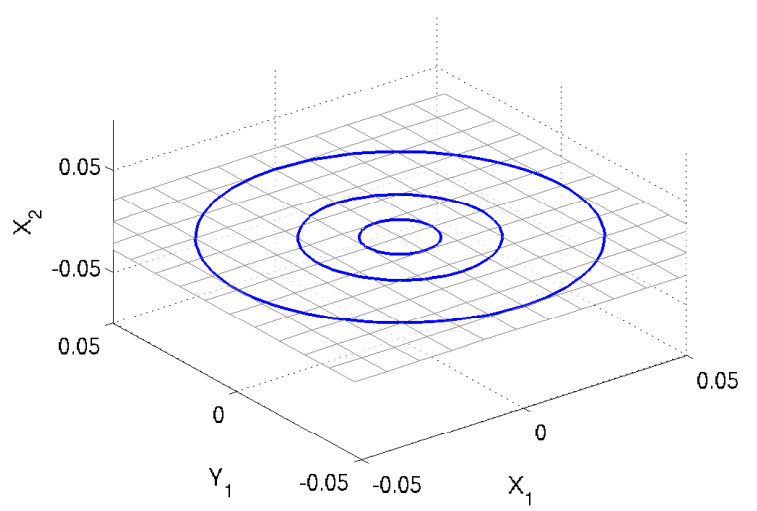

Figure 4: Trajectories (closed periodic orbits) of the linear system, represented in space $\left(X_{1}, Y_{1}, X_{2}\right)$. Motions along the first linear mode with initial conditions $X_{1}(t=0)=0.01,0.025$ and 0.05 . The linear eigenspace is represented by the horizontal plane $X_{2}=0$. The trajectories initiated along the linear mode stay within for al time $t$ (invariance property).

so that illustration of the complete phase space is not possible. Figure 4 shows, in a selected subspace $\left(X_{1}, Y_{1}, X_{2}\right)$, with $Y_{1}=\dot{X}_{1}$, three trajectories of the corresponding linear system, where quadratic and cubic terms have been cancelled. The linear modes of the system corresponds to purely vertical and 
purely horizontal motions of the mass. In phase space, the linear eigenspaces are the two-dimensional planes, respectively defined by $X_{2}=Y_{2}=0$ for the first mode, and $X_{1}=Y_{1}=0$ for the second mode. The three trajectories have been computed for three initial conditions $X_{1}(t=0)=0.01,0.025$ and 0.05 (all other coordinates equal to zero). As awaited for the linear system, the motion initiated in the first linear mode stay in this plane for all time $t$ : this is the invariance property, that results from the decoupling of the system at the linear stage.

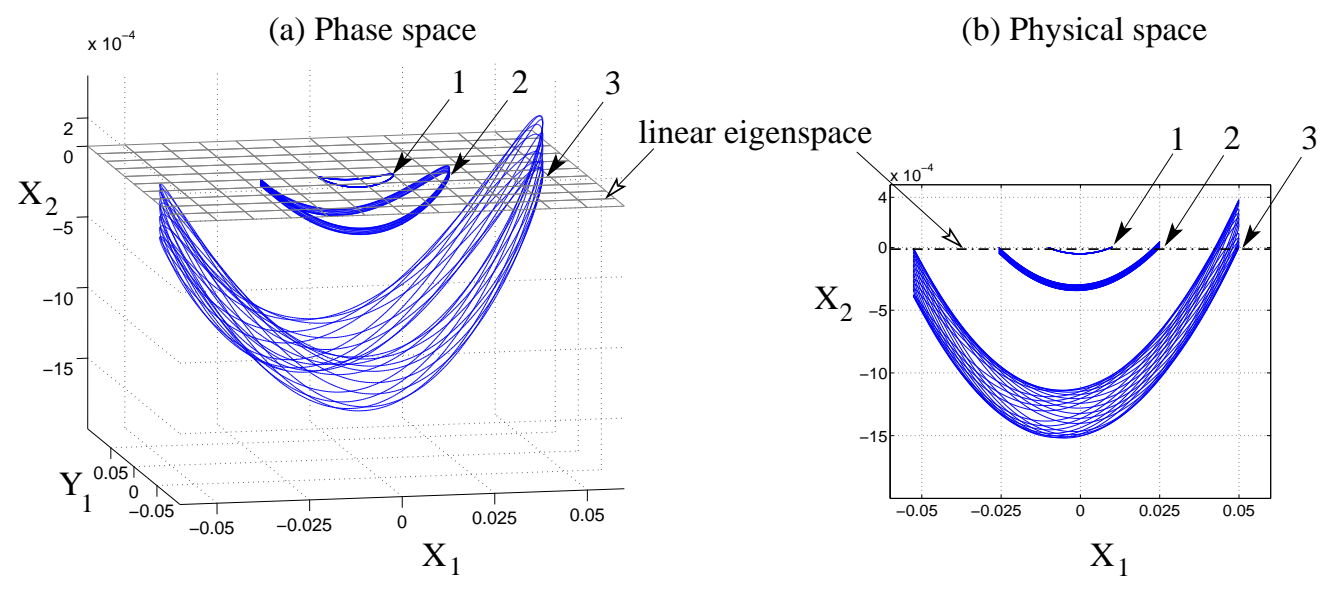

Figure 5: Trajectories of the nonlinear system, Eqs. (42). Motions initiated in the first linear mode with initial conditions $X_{1}(t=0)=0.01,0.025$ and 0.05 (all other coordinates to zero), illustrating the non-invariance of the linear eigenspace for the nonlinear system.

Let us consider now the complete nonlinear system. We repeat the same numerical experiment, that is to say we compute the three trajectories corresponding to an initial condition in the first linear mode with increasing amplitude $X_{1}(t=0)=0.01,0.025$ and 0.05 . The corresponding trajectories are reported in Fig. 5. As the first one with the smallest amplitude stay in the vicinity of the linear eigenespace, it is very clear from the other two that the invariance property is not fulfilled anymore, as the trajectories are whirling around the linear eigenspace. It is worth mentionable that this behaviour only results on the presence of the invariant-breaking term $X_{1}^{2}$ on the second oscillator-equation (42b): as energy is fed on the first oscillator along the first linear mode, the term $X_{1}^{2}$ transfers a small amount of energy and excites the oscillations around the second coordinate, thus violating the invariance property.

Invariance can be recovered by selecting another set of initial conditions. Figure 6 shows the trajectories computed for $\left(X_{1}, X_{2}\right)=(0.01,0) ;\left(0.025,2.3^{*} 10^{-5}\right)$ and $\left(0.05,1.8^{*} 10^{-4}\right)$, i.e. the same amplitude for $X_{1}$ but with a small, selected perturbation on $X_{2}$, which allows recovering closed periodic orbits. The figure illustrates the dual definition of a NNM for conservative system, which can be viewed either as a family of periodic orbits, or as an invariant manifold, tangent at origin to the corresponding linear eigenspace.

As noted previously, the invariant-breaking terms are cancelled with the change of coordinates of the normal transformation, as long as no internal resonances are present between the eigenfrequencies. Fig. 7 presents a schematical representation of the phase space and a geometrical interpretation of the normal form transformation. As expressed with the modal coordinates, the equations of motion are written in a phase space which is spanned by the linear eigenspaces (two-dimensional planes parameterized by $\left(X_{p}, Y_{p}\right)$ ). Due to the presence of invariant-breaking terms, when the dynamics is expressed with these coordinates, linear modes are not invariant in the nonlinear regime. To recover invariance, the idea is to span the phase space with the NNMs (the invariant manifold) of the system, sketched as $\mathcal{M}_{1}$ and $\mathcal{M}_{2}$ in Fig. 7. The normal transformation is the key for expressing the new, normal coordinates (linked to the invariant manifolds), to the original, modal ones. The transformation is nonlinear, expressing the fact that the invariant manifolds are curved subspaces. The nonlinear transform cancels the invariant-breaking 


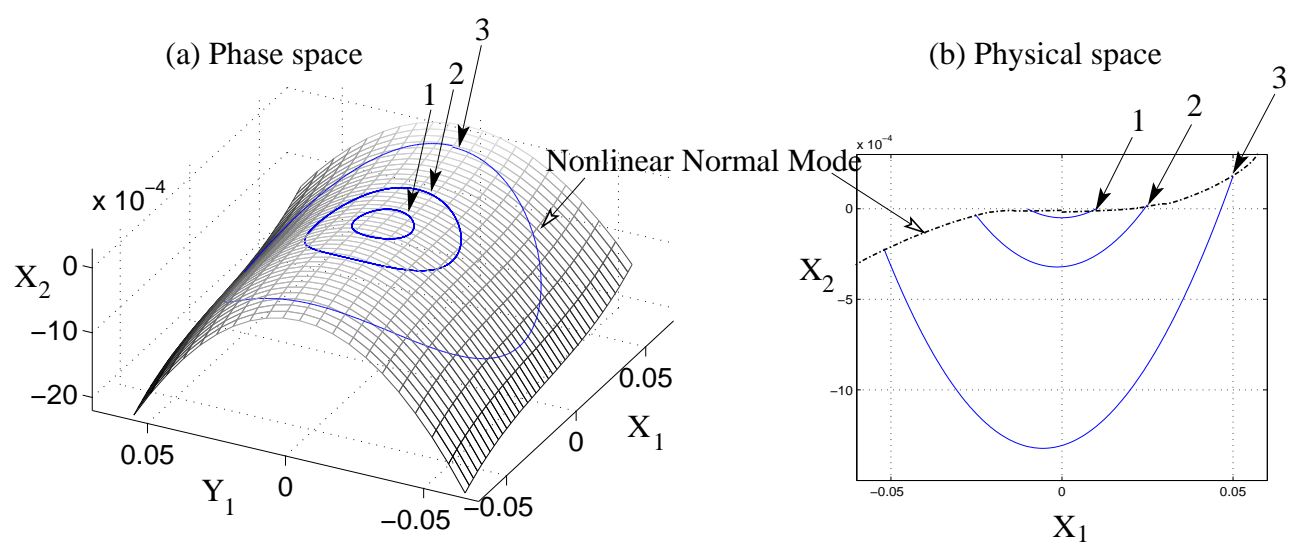

Figure 6: Trajectories (closed periodic orbits) of the nonlinear system, Eqs. (42). Motions initiated in the first nonlinear normal mode with initial conditions $\left(X_{1}, X_{2}\right)=(0.01,0) ;\left(0.025,2.3^{*} 10^{-5}\right)$ and $(0.05$, $1.8^{*} 10^{-4}$ ). Shaded surface: invariant manifold (first NNM).

terms. The dynamics, expressed with the normal coordinates, is hence written in a curved invariant-based span of the phase space.

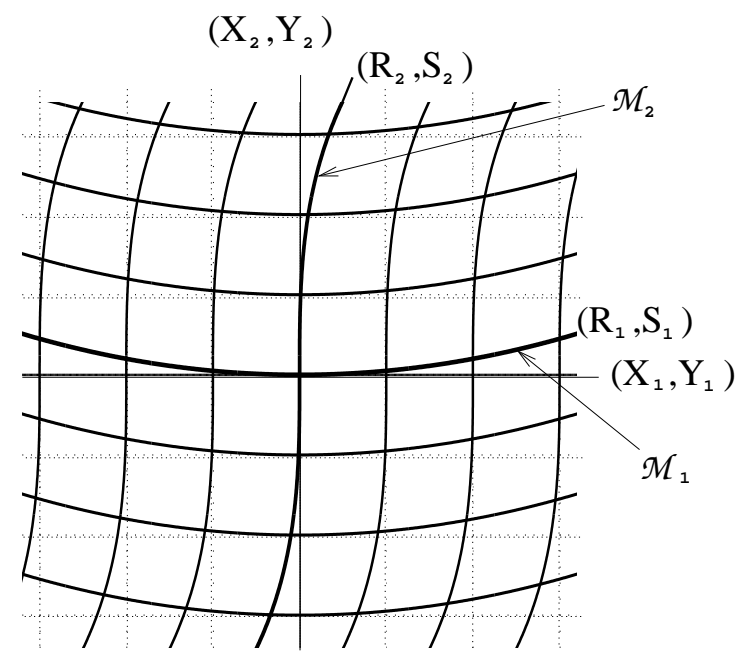

Figure 7: Schematic representation of a four-dimensional phase space for illustrating the normal transform. Two-dimensional manifolds rare represented by lines. $\left(X_{1}, X_{2}, Y_{1}, Y_{2}\right)$ : modal coordinates, cartesian grid: mesh generated by the linear eigenspace parameterization. $\mathcal{M}_{1}, \mathcal{M}_{2}$ : invariant manifolds. $\left(R_{1}, R_{2}, S_{1}, S_{2}\right)$ : normal coordinates. Curved grid associated to the invariant-based span of the phase space.

The invariance property is key for allowing one to operate proper truncations. Indeed, when working out with real systems emanating from the discretization of continuous structures, one is led to manipulate an a priori infinite number of modes which must be truncated for numerical treatments. The results shown here with a simple two-dofs system can easily extend to systems with numerous dofs that can be decomposed in subgroups. Due to the presence of invariant-breaking nonlinear terms, truncations on the dynamics expressed with the modal coordinates leads to simulate trajectories that do not exist in the complete phase space. On the other hand, once the dynamics expressed in the curved, invariant-based span, proper truncations can be realized, as the trajectories simulated with the truncated system corre- 
sponds to those of the complete system. This remark is very important and will be the key to derive accurate reduced-order models that are able $e . g$. to predict the correct type of nonlinearity for a given set of coupled oscillators.

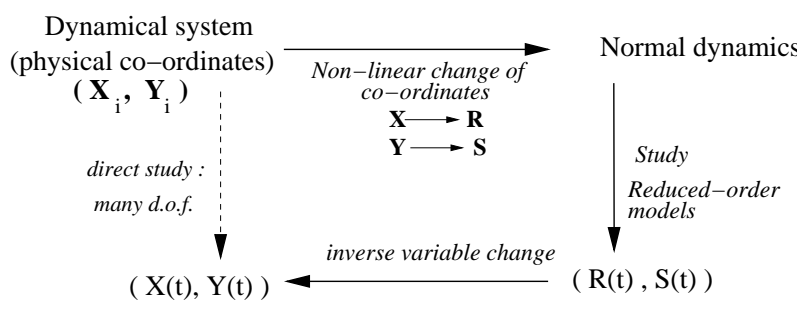

Figure 8: Illustration of the nonlinear transform for derivation of reduced-order models.

Fig. 8 illustrates the general idea for deriving accurate reduced-order models (ROMs) thanks to normal form theory. As compared to reduction methods derived by using a linear change of coordinates (e.g. projection onto the linear mode basis, or Proper Orthogonal Decomposition (POD) method), the idea here is to use a nonlinear change of coordinates. Once the system expressed in its normal form, the truncations can be realized as one is ascertained of an accurate and meaningful result thanks to the invariance property. In that respect, the method proposed here can thus be sumed up as using a more sophisiticated, nonlinear transformation, before realizing the truncations.

The next subsection considers the simplest case where the motion is reduced to a single NNM. More complicated cases involving internal resonances are handled in subsection 2.7, as well as in section 4 with applications to shells.

\subsection{Single-mode motion}

In order to restrict the dynamics to a single NNM, one has just to proceed as usual with the normal coordinates. As invariance is recovered, to study e.g. $p^{\text {th }} \mathrm{NNM}$, all the other coordinates $k \neq p$ need to be cancelled:

$$
\forall k \neq p: R_{k}=S_{k}=0,
$$

Susbtituting for (43) in (39) gives the geometry of the manifold in phase space (approximated at third order):

$$
\begin{aligned}
& \forall k \neq p: \\
& \quad X_{k}=a_{p p}^{k} R_{p}^{2}+b_{p p}^{k} S_{p}^{2}+r_{p p p}^{k} R_{p}^{3}+u_{p p p}^{k} R_{p} S_{p}^{2}, \\
& Y_{k}=\gamma_{p p}^{k} R_{p} S_{p}+\mu_{p p p}^{k} S_{p}^{3}+\nu_{p p p}^{k} S_{p} R_{p}^{2} .
\end{aligned}
$$

This set of $2(N-1)$ equations in a phase space of dimension $2 N$ defines the geometry of the invariant manifold, up to third order. Detailed comparisons with other computations led by different authors, have been realized. In particular the invariant manifold procedure proposed by S. Shaw and C. Pierre in [42] and its asymptotic development for solving out the Partial Differential Equation defining the geometry resolved in [37, 38] gives exactly the same expressions. The same comparison has also been realized with the NNM computation procedure with a multiple scale approach, as proposed by Nayfeh [34], and once again all coefficients match, evidencing the equivalence of the different methods.

The dynamics onto the $p^{\text {th }}$ manifold is found by substituting for (43) in (40), and reads

$$
\ddot{R}_{p}+\omega_{p}^{2} R_{p}+\left(A_{p p p}^{p}+h_{p p p}^{p}\right) R_{p}^{3}+B_{p p p}^{p} R_{p}{\dot{R_{p}}}^{2}=0 .
$$


This procedure for reducing the dynamics to selected invariant subspaces, is the key for deriving out proper reduced-order models that will be able to reproduce all the qualitative and quantitative features of the complete system. This point will be key for the remainder of the presentation. Note that the normal form theory provides the most complete picture for expressing the NNMs of a system, as the nonlinear change of coordinate (39) is a complete change, from the phase space into itself. It contains the geometry of the $N$ NNMs that are tangent at origin to the linear eigenspaces, and thus can be viewed as "extensions" of the linear modes. For recovering results obtained with other methods, e.g. [42, 34] from normal form theory, one has to restrict the results to particular cases. On the other hand, extending the result obtained via, e.g. the invariant manifold method, to more than one dof (at best $N$ ) is generally a very tedious task $[37,23]$.

\subsection{Classification of nonlinear terms, case of internal resonance}

From all the results obtained in the previous subsection, one is now able to draw out a classification of the nonlinear coupling terms appearing in the equations of motion, in order to get a clear physical understanding of their meaning on the dynamics. For that purpose, let us consider a general, two dofs system with quadratic and cubic nonlinearities:

$$
\begin{aligned}
\ddot{X}_{1}+\omega_{1}^{2} X_{1} & +g_{11}^{1} X_{1}^{2}+g_{12}^{1} X_{1} X_{2}+g_{22}^{1} X_{2}^{2} \\
& +h_{111}^{1} X_{1}^{3}+h_{112}^{1} X_{1}^{2} X_{2}+h_{122}^{1} X_{1} X_{2}^{2}+h_{222}^{1} X_{2}^{3}=0 \\
\ddot{X}_{2}+\omega_{2}^{2} X_{2} & +g_{11}^{2} X_{1}^{2}+g_{12}^{2} X_{1} X_{2}+g_{22}^{2} X_{2}^{2} \\
& +h_{111}^{2} X_{1}^{3}+h_{112}^{2} X_{1}^{2} X_{2}+h_{122}^{2} X_{1} X_{2}^{2}+h_{222}^{2} X_{2}^{3}=0
\end{aligned}
$$

and let us focus on the first oscillator equation. The following classification can be derived from the precedent results:

- The cubic terms $X_{1}^{3}$ and $X_{1} X_{2}^{2}$ are trivially resonant terms. they can not be cancelled by the change of coordinate. Note that when no internal resonance of order two are present, all the quadratic terms can be cancelled by the normal transform.

- All the other terms can be cancelled by the normal transform. Among them, the terms $X_{2}^{2}$ and $X_{2}^{3}$ are invariant-breaking terms: they are source terms that couple equations 1 and 2 , whatever a resonance relationship between the eigenfrequencies exist or not. If no internal resonance exist, they are coined non-resonant coupling terms, otherwise the coupling is stronger, energy is exchanged between the modes and the terms are coined resonant coupling terms.

Let us consider now the case of internal resonance to explain how the precedent results must be adapted. Let us assume for illustration that a relationship $\omega_{2}=2 \omega_{1}$ exist between the two eigenfrequencies. This is a second-order resonance relationship, which implies that quadratic terms won't be cancellable by the normal transform. To recover the resonant coupling terms that will stay in the normal form (without making the complete calculation), the following rule of thumb can be adopted. At the linear stage, the solution for $X_{1}$ and $X_{2}$ reads: $X_{1} \sim \exp \pm i \omega_{1} t, X_{2} \sim \exp \pm i \omega_{2} t$. The nonlinear term $X_{1}^{2}$ behaves as (amongts other solutions) $X_{1}^{2} \sim \exp 2 i \omega_{1} t=\exp i \omega_{2} t$. Hence this term can be viewed as a forcing term for the second oscillator equation, which is moreover exactly tuned at its eigenfrequency, and thus will lead to a resonance and the appearance of secular terms. This resonance is the key for explaining why a small denominator problem appear when computing the normal form, so that this term can not be removed. Following the same reasoning and considering now the monom $X_{1} X_{2}$, one can see that this term can produce oscillatory motions like $X_{1} X_{2} \sim \exp i\left(\omega_{2}-\omega_{1}\right) t=\exp i \omega_{1} t$. hence this term is a forcing term acting at the resonance frequency of the first oscillator, thus it will stay in the first oscillator-equation in the normal form. As a conclusion, for that case $\omega_{2}=2 \omega_{1}$, the normal form up to 
quadratic term reads:

$$
\begin{aligned}
& \ddot{X}_{1}+\omega_{1}^{2} X_{1}+g_{12}^{1} X_{1} X_{2}=0, \\
& \ddot{X}_{2}+\omega_{2}^{2} X_{2}+g_{11}^{2} X_{1}^{2}=0,
\end{aligned}
$$

At third order, the internal resonance $\omega_{2}=2 \omega_{1}$ do not create new resonance condition, so that the cubic terms can be treated as usual. the only difficulty is to track to nonlinear coefficient of the normal form that corresponds to the monoms which have not been cancelled. These terms are easily found by looking to the formulas given in Appendix A. Then the complete normal form up to order three can easily be written.

This example shows that the treatment of internal resonance is not made too difficult with the formalism of normal form, contrary to the huge complexities involved in other methods (invariant manifold, multiple scales) to adapt their treatments to the case of internal resonance.

\subsection{Damped systems}

All the developments presented in the previous section have been obtained under the assumption of a conservative system. Obviously when one deals with real structures, damping is at hand and should be considered, in particular in the development of accurate reduced-order models. A simple strategy is to build the ROM without considering the damping, with the nonlinear change of coordinate presented in subsection 2.4. Then damping could be added on the normal form dynamics, i.e. on the reduced order model, directly. However, numerical examples have shown that this method underestimates the whole damping present in the structure $[50]^{3}$. This underestimation is a consequence of the fact that a single NNM merges the contributions of numerous linear normal modes. When modal damping is added to each linear contribution, the decay rate of all the linear modes that are gathered to create the selected NNM are somehow added, so that the decay rate onto the manifold is not as simple as the initial decay rate postulated for the linear modal coordinates. Hence the normal form strategy must be adapted to handle the case of damped systems. The presentation now follows closely the results published in [50].

The starting point is now an assembly of $N$ oscillator-equations, expressed within the modal basis, so that the linear coupling terms are diagonal. A modal damping is assumed so that the dynamics reads, $\forall p=1 \ldots N$ :

$$
\ddot{X}_{p}+\omega_{p}^{2} X_{p}+2 \xi_{p} \omega_{p} \dot{X}_{p}+\sum_{i=1}^{N} \sum_{j \geq i}^{N} g_{i j}^{p} X_{i} X_{j}+\sum_{i=1}^{N} \sum_{j \geq i}^{N} \sum_{k \geq j}^{N} h_{i j k}^{p} X_{i} X_{j} X_{k}=0 .
$$

Deriving a correct mechanical model of damping (including thermoelasticity, viscoelasticity, fluid-structure interaction...) for a large class of structure is an extremely difficult task, which also greatly depends on some specific properties of the material used. The great majority of studies on vibrations of continuous structures uses an ad-hoc viscous modal damping as the one which is here postulated. It is assumed that the modal damping introduced gives an excellent approximation of the energy losses in the considered structure, and has been finely tuned for each mode by any available method (numerical prediction or experimental fitting). Underdamped eigenmodes, corresponding to oscillatory motions, are considered, so that: $\forall p=1 \ldots N: \xi_{p}<1$.

Being a linear term, the modal viscous damping has an effect on the eigenvalues of the structures. For mode $p$, the two complex conjugated eigenvalues reads (where $i$ is such that $i^{2}=-1$ ):

$$
\lambda_{p}^{ \pm}=-\xi_{p} \omega_{p} \pm i \omega_{p} \sqrt{1-\xi_{p}^{2}}
$$

\footnotetext{
${ }^{3}$ These examples will be shown in section 4 .
} 
Besides the real part of Eq. (49) which controls the decay rate of energy along the $p^{\text {th }}$ linear eigenspace, the imaginary part shows that the damping also have an effect on the oscillation frequency. For numerous thin metallic structures, the damping ratio $\xi_{p}$ is very small for all $p$, so that the assumption of a lightly damped system could be considered. In that case, a first-order development of (49) shows that:

$$
\lambda_{p}^{ \pm} \approx \pm i \omega_{p}-\xi_{p} \omega_{p}+\mathcal{O}\left(\xi_{p}^{2}\right)
$$

which shows that the correction on the frequency is at least a second-order effect. For computing the normal form, the general formalism can be adopted, excepting that now the eigenvalues are complex number with real and imaginary parts. At first sight, the occurrence of the real part could let us think that the trivial resonance relationships would be destroyed, as they were a simple consequence of having a purely imaginary eigenspectrum. However, the constraint that the normal form computed for the damped system is an extrapolation of the undamped system, is assumed. This implies that, by continuity, when the damping ratio $\xi_{p}$ tends to zero, the new calculation must recover that obtained for the undamped system. In particular, this condition involves that small denominator of the form $1 / \xi_{p}$ are not allowed in the calculation. Interestingly, these small denominators appears only for the trivially resonant terms. Hence all the calculations are led with the extra condition that when a coefficient in the normal form transform scales as $1 / \xi_{p}$, then it must be cancelled, and the corresponding monom stay in the normal form. By doing so, only the trivially resonant terms are kept, and a continuity from undamped to damped real normal forms, is obtained.

The calculation proceeds in the same manner as in the conservative case. It is still assumed that no internal resonance are at hand ${ }^{4}$ (the case of internal resonance being easily treated as shown in subsection 2.7). The non-linear change of co-ordinates reads:

$$
\begin{gathered}
X_{p}=R_{p}+\sum_{i=1}^{N} \sum_{j \geq i}^{N}\left(a_{i j}^{p} R_{i} R_{j}+b_{i j}^{p} S_{i} S_{j}\right)+\sum_{i=1}^{N} \sum_{j=1}^{N} c_{i j}^{p} R_{i} S_{j}+\sum_{i=1}^{N} \sum_{j \geq i}^{N} \sum_{k \geq j}^{N}\left(r_{i j k}^{p} R_{i} R_{j} R_{k}+s_{i j k}^{p} S_{i} S_{j} S_{k}\right) \\
+\sum_{i=1}^{N} \sum_{j=1}^{N} \sum_{k \geq j}^{N}\left(t_{i j k}^{p} S_{i} R_{j} R_{k}+u_{i j k}^{p} R_{i} S_{j} S_{k}\right), \\
Y_{p}=S_{p}+\sum_{i=1}^{N} \sum_{j \geq i}^{N}\left(\alpha_{i j}^{p} R_{i} R_{j}+\beta_{i j}^{p} S_{i} S_{j}\right)+\sum_{i=1}^{N} \sum_{j=1}^{N} \gamma_{i j}^{p} R_{i} S_{j}+\sum_{i=1}^{N} \sum_{j \geq i}^{N} \sum_{k \geq j}^{N}\left(\lambda_{i j k}^{p} R_{i} R_{j} R_{k}+\mu_{i j k}^{p} S_{i} S_{j} S_{k}\right) \\
+\sum_{i=1}^{N} \sum_{j=1}^{N} \sum_{k \geq j}^{N}\left(\nu_{i j k}^{p} S_{i} R_{j} R_{k}+\zeta_{i j k}^{p} R_{i} S_{j} S_{k}\right)
\end{gathered}
$$

The normal dynamics can thus be explicitely written: $\forall p=1 \ldots N$ :

$$
\begin{aligned}
& \dot{R}_{p}=S_{p} \\
& \begin{aligned}
\dot{S}_{p}= & -\omega_{p}^{2} R_{p}-2 \xi_{p} \omega_{p} S_{p}-\left(h_{p p p}^{p}+A_{p p p}^{p}\right) R_{p}^{3}-B_{p p p}^{p} R_{p} S_{p}^{2}-C_{p p p}^{p} R_{p}^{2} S_{p} \\
- & R_{p}\left[\sum_{j>p}^{N}\left[\left(h_{p j j}^{p}+A_{p j j}^{p}+A_{j p j}^{p}\right) R_{j}^{2}+B_{p j j}^{p} S_{j}^{2}+\left(C_{p j j}^{p}+C_{j p j}^{p}\right) R_{j} S_{j}\right]\right. \\
& \left.\quad+\sum_{i<p}\left[\left(h_{i i p}^{p}+A_{i i p}^{p}+A_{p i i}^{p}\right) R_{i}^{2}+B_{p i i}^{p} S_{i}^{2}+\left(C_{p i i}^{p}+C_{i p i}^{p}\right) R_{i} S_{i}\right]\right] \\
& -S_{p}\left[\sum_{j>p}^{N}\left(B_{j p j}^{p} R_{j} S_{j}+C_{j j p}^{p} R_{j}^{2}\right)+\sum_{i<p}\left(B_{i i p}^{p} R_{i} S_{i}+C_{i i p}^{p} R_{i}^{2}\right)\right]
\end{aligned}
\end{aligned}
$$

\footnotetext{
${ }^{4}$ Once again, for obtaining continuity with the conservative case, internal resonance relationships are defined here only for the imaginary part (frequency) of the eigenvalues.
} 
The coefficients $\left(A_{i j k}^{p}, B_{i j k}^{p}, C_{i j k}^{p}\right)$ arise from the cancellation of the quadratic terms. Their expressions are:

$$
\begin{aligned}
A_{i j k}^{p} & =\sum_{l \geq i}^{N} g_{i l}^{p} a_{j k}^{l}+\sum_{l \leq i} g_{l i}^{p} a_{j k}^{l}, \\
B_{i j k}^{p} & =\sum_{l \geq i}^{N} g_{i l}^{p} b_{j k}^{l}+\sum_{l \leq i} g_{l i}^{p} b_{j k}^{l} . \\
C_{i j k}^{p} & =\sum_{l \geq i}^{N} g_{i l}^{p} c_{j k}^{l}+\sum_{l \leq i} g_{l i}^{p} c_{j k}^{l} .
\end{aligned}
$$

As compared to the conservative case, introducing the damping in the linear operator leads to a nonlinear change of co-ordinates, Eq. (39), which is now complete (in terms of the monoms $\left(R_{i}, S_{j}\right)$ ). The newly introduced coefficients: $\left\{c_{i j}^{p}, \alpha_{i j}^{p}, \beta_{i j}^{p}, s_{i j k}^{p}, t_{i j k}^{p}, \lambda_{i j k}^{p}, \zeta_{i j k}^{p}\right\}$ bring a perturbation which is at least of the order of the damping ratios $\left\{\xi_{i}\right\}$. More precisely, Eq. (39) may be expanded as a power series of the small perturbative terms $\left\{\xi_{i}\right\}$. It is then found that the coefficients that were non-zero in the conservative case (i.e. $\left.\left\{a_{i j}^{p}, b_{i j}^{p}, \gamma_{i j}^{p}, r_{i j k}^{p}, u_{i j k}^{p}, \mu_{i j k}^{p}, \nu_{i j k}^{p}\right\}\right)$ contains only even powers of the damping ratios, and the new terms, $\left\{c_{i j}^{p}, \alpha_{i j}^{p}, \beta_{i j}^{p}, s_{i j k}^{p}, t_{i j k}^{p}, \lambda_{i j k}^{p}, \zeta_{i j k}^{p}\right\}$, contains only odd powers of the damping ratios. As a consequence, the $\left\{A_{i j k}^{p}, B_{i j k}^{p}, C_{i j k}^{p}\right\}$ terms defined in Eqs. (53), can also be expanded as power series of the damping ratios. It is then found that $\left\{A_{i j k}^{p}, B_{i j k}^{p}\right\}$ contains only even powers of the damping ratios and may be sorted according to $\mathcal{O}\left(\xi_{i}^{0}\right), \mathcal{O}\left(\xi_{i}^{2}\right), \mathcal{O}\left(\xi_{i}^{4}\right)$,... So that, in the limit of a conservative systems, $A_{i j k}^{p}$ and $B_{i j k}^{p}$ tends to a non-zero value. On the other hand, $C_{i j k}^{p}$ sorts according to odd powers terms: $\mathcal{O}\left(\xi_{i}^{1}\right), \mathcal{O}\left(\xi_{i}^{3}\right), \mathcal{O}\left(\xi_{i}^{5}\right), \ldots$ So that it is equal to zero in the conservative case. Hence this "damped" formulation allows one to see the damped normal dynamics as a perturbation of the undamped one, and could be use in great generality as the conservative results are recovered by simply cancelling all the $\xi_{p}$.

Let us comment some of the differences brought by this new formulation. To have a better picture, let us restrict ourselve to studying a single NNM, labelled $p$ (master mode), obtained by letting $\forall k \neq$ $p, R_{k}=S_{k}=0$ in the previous expressions. The geometry of the $p^{\text {th }}$ manifold in phase space now reads:

$$
\begin{aligned}
& \forall k \neq p: \\
& \qquad \begin{array}{l}
X_{k} \\
=a_{p p}^{k} R_{p}^{2}+b_{p p}^{k} S_{p}^{2}+c_{p p}^{k} R_{p} S_{p}+r_{p p p}^{k} R_{p}^{3}+s_{p p p}^{k} S_{p}^{3}+t_{p p p}^{k} R_{p}^{2} S_{p}+u_{p p p}^{k} R_{p} S_{p}^{2}, \\
Y_{k}=\alpha_{p p}^{k} R_{p}^{2}+\beta_{p p}^{k} S_{p}^{2}+\gamma_{p p}^{k} R_{p} S_{p}+\lambda_{p p p}^{k} R_{p}^{3}+\mu_{p p p}^{k} S_{p}^{3}+\nu_{p p p}^{k} R_{p}^{2} S_{p}+\zeta_{p p p}^{k} R_{p} S_{p}^{2} .
\end{array}
\end{aligned}
$$

As compared to the conservative case, Eq. (44), new coefficients (and thus new monoms) appears. Secondly the already present coefficients, e.g. $a_{p p}^{k}, b_{p p}^{k}$, now depends on the damping values. An illustration of their dependence is shown in Fig. 9, obtained for the two-dofs system presented in subsection 2.5. The equations of motion are given by Eqs. (42), and modal damping of the form $2 \xi_{p} \omega_{p} \dot{X}_{p}$, is added on each equation, for $p=1,2$. The parameters have been set to $\omega_{1}=2, \omega_{2}=4.5$. The first NNM is selected, so that $p=1$ and $k=2$. The figure shows the variation of the quadratic coefficients of the first equation, $a_{11}^{2}$, $b_{11}^{2}$ and $c_{11}^{2}$, for two different cases. In Fig. 9(a), the two damping coefficients have the same values, so that $\xi_{1}=\xi_{2}=\xi$, and $\xi$ is increased from 0 to 0.4 , so that a system that is more and more damped is studied. When $\xi_{1}=\xi_{2}=0$, the conservative results are recovered: $c_{11}^{2}$ is equal to zero whereas $a_{11}^{2}, b_{11}^{2}$ have a nonzero value. One can see that the variations may be large for some of the coefficients, so that the geometry of the manifold can be significantly changed when damping is increased. In the second case, Fig. 9(b), the damping on the first linear mode is selected at a constant, small value, $\xi_{1}=10^{-3}$, and $\xi_{2}$ is raised from 0 to 0.4 in order to simulate a situation where the slave mode is more and more damped, as compared to the master. Here the variations are also found to be important for large values of the damping. 

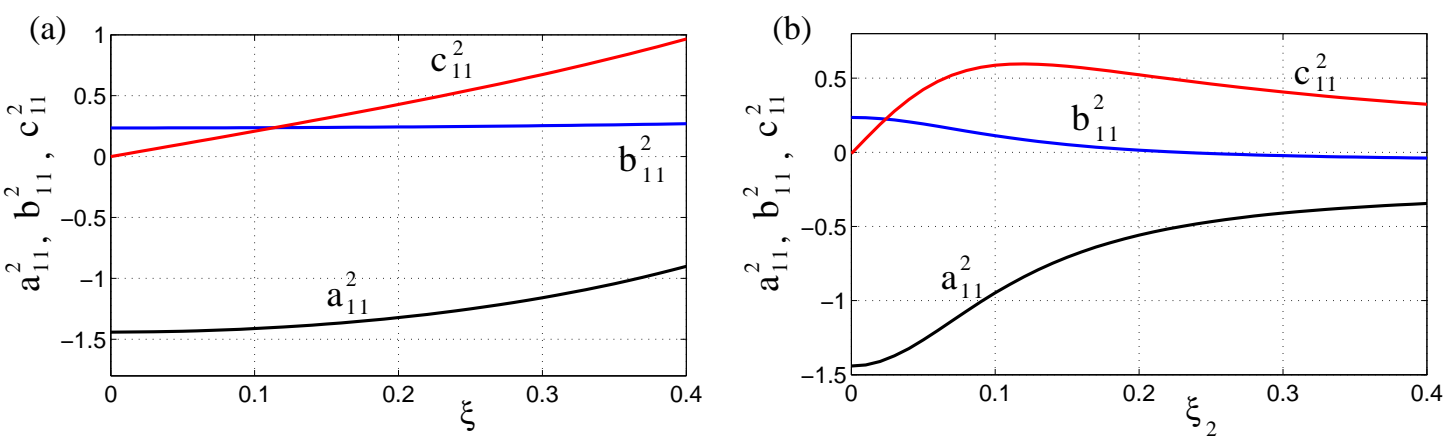

Figure 9: Variarions of the coefficients $a_{11}^{2}, b_{11}^{2}$ and $c_{11}^{2}$, controlling the quadratic part of the geometry of the first NNM, as functions of the damping ratios, for the two-dofs system. Parameters are: $\omega_{1}=2$, $\omega_{2}=4.5$. (a): variations for an increasing value of the two damping ratios taken as equal: $\xi_{1}=\xi_{2}=\xi$. (b): variations for a fixed value $\xi_{1}=10^{-3}$ and an increasing $\xi_{2}$.

The normal dynamics for a single NNM motion when damping is included reads:

$$
\ddot{R}_{p}+\omega_{p}^{2} R_{p}+2 \xi_{p} \omega_{p} \dot{R}_{p}+\left(h_{p p p}^{p}+A_{p p p}^{p}\right) R_{p}^{3}+B_{p p p}^{p} R_{p} \dot{R}_{p}^{2}+C_{p p p}^{p} R_{p}^{2} \dot{R}_{p}=0 .
$$

The new coefficients appearing in this equation, as compared to Eq. (45), is $C_{p p p}^{p}$. Eq. (53c) reveals that $C_{p p p}^{p}$ is constructed from all the values of the $c_{p p}^{k}, \mathrm{k}=1 \ldots \mathrm{N}$, that also directly influences the geometry of the manifold, Eq. (54a). The corresponding monom, $R_{p}^{2} \dot{R}_{p}$, can be interpreted as a nonlinear damper. Hence the whole damping for the dynamics on the $p^{\text {th }}$ NNM gathers a linear damping term, $2 \xi_{p} \omega_{p} \dot{R}_{p}$, defining the energy decay rate onto the linear mode (remember that the change of coordinate is identitytangent), and a nonlinear damping term, $C_{p p p}^{p} R_{p}^{2} \dot{R}_{p}$, allowing for a more precise definition of the global decay rate onto the manifold, taking into account all damping terms of the linear contributions that are included in the construction of the NNM. The coefficients $A_{p p p}^{p}$ and $B_{p p p}^{p}$, now depends on the damping in a manner that is proportional to $a_{p p}^{k}$ and $b_{p p}^{k}$, so that their variations can be inferred from Fig. 9. These coefficients are responsible for the type of nonlinearity (hardening/softening behaviour) of the NNM, so a more complete study is postponed to the next section which is entirely devoted to this problematic.

\subsection{Closing remarks}

The main theoretical results for deriving a normal form approach for nonlinear normal modes, has been shown in this section. The remainder of the lecture is devoted to applications of the results obtained. Some of the assumptions made in this section can be easily relaxed to extend the generality of the results. In particular, the special case of structural systems with quadratic and cubic nonlinearities, Eqs. (48), has been here assumed as it contains the great majority of applications to thin structures like beams, plates and shells, vibrating at large amplitudes. However, more general cases including for example nonlinear damping monoms of the form $\dot{X}_{i} X_{j}$ (quadratic) or $\dot{X}_{i} X_{j} X_{k}, \dot{X}_{i} \dot{X}_{j} X_{k}$ are of course amenable to a solution following the same steps. Another limitation could appear through the asymptotic develoments, systematically stopped at third order. Once again, this limitation can be overcome by pushing further the developments, which is not a theoretical problem, but may appear as more and more difficult, due to the complexity one has to faced, e.g. by pushing up to order 5. Moreover, the legitimate question is to know if the game is worth the candle. Asymptotic expansions up to order five realized for computing the NNMs with the center manifold technique in [43] show that the gain in accuracy brought by the fifth-order is not significative, and in some cases can be poorest than the third-order. Closer investigations on the validity limits of normal form approach in [28], as well as thorough comparisons between asymptotics and numerical computation of NNMs in [10], appears to reach the same conclusion, 
that is to say that pushing further the asymptotics may lead to small improvments as compared to the computational effort needed. In the remainder of the lecture, the third-order expansion will thus always be used for applications.

\section{Hardening/softening behaviour}

This section is entirely devoted to the correct prediction of the type of nonlinearity (hardening/softening behaviour) for the modes of an assembly of $N$ nonlinear oscillator equations as in Eq. (2). NNMs and normal form are used to derive a proper, easy-to-use and almost analytical method. The presentation in subsections 3.1 and 3.2 recalls the main results obtained in [55]. The next subsection 3.3 with applications to shells gives the main results published in $[53,47]$, while subsection 3.4 gathers important results published in [50] on the influence of the damping.

\subsection{Definition}

In nonlinear vibrations, the oscillation frequencies of a system depend on vibration amplitude, a feature that has no counterpart in linear theory. This dependence can be of two different types. Either a hardening behaviour is at hand, which implies that the oscillation frequency increases with the amplitude, or a softening type nonlinearity is present, which means that the oscillation frequency decreases with the amplitude. This behaviour can be easily illustrated with the Duffing oscillator:

$$
\ddot{X}+\omega_{0}^{2} X+\alpha X^{3}=0 .
$$

A perturbation method (multiple scales, Poincaré-Lindstedt, Averaging, ...) can be used in order to derive the first-order relationship between the nonlinear oscillation frequency, denoted $\omega_{N L}$, and the amplitude $a$ of the oscillation frequency, which reads: $X(t)=a \cos \left(\omega_{N L} t+\phi\right)+\mathcal{O}\left(a^{2}\right)$. We obtain:

$$
\omega_{N L}=\omega_{0}\left(1+\frac{3 \alpha}{8 \omega_{0}^{2}} a^{2}\right) .
$$

This equation clearly shows that a hardening behaviour is obtained when $\alpha>0$, which also explains the origin of the vocable "hardening": when $\alpha>0$, the nonlinear stiffness of the oscillator can be written as $\omega_{0}^{2} X\left(1+\frac{\alpha}{\omega_{0}^{2}} X^{2}\right)$, which means that the more $X$ is large (the particle is far from the equilibrium position), the more the spring is stiff: the stiffness term has thus a "hardening behaviour". On the other hand when $\alpha<0$, the more $X$ is large the less the restoring force is important, and consequently the oscillation frequency decreases with vibration amplitude.

Let us consider now the case of a single nonlinear oscillator with quadratic and cubic nonlinearities. We can for example assume that the system (2) has been reduced to a single linear mode, say number $p$, and thus the dynamics reads:

$$
\ddot{X}_{p}+\omega_{p}^{2} X_{p}+g_{p p}^{p} X_{p}^{2}+h_{p p p}^{p} X_{p}^{3}=0 .
$$

The frequency-amplitude relationship in this case writes:

$$
\omega_{N L}=\omega_{0}\left(1+\tilde{\Gamma}_{p} a^{2}\right), \quad \text { with } \quad \tilde{\Gamma}_{p}=\frac{1}{8 \omega_{p}^{2}}\left(3 h_{p p p}^{p}-\frac{10 g_{p p}^{p}{ }^{2}}{3 \omega_{p}^{2}}\right) .
$$

One can see that the quadratic and cubic coefficients have opposite effects on the type of nonlinearity.

However, as it has been shown previously, linear eigenspaces are not invariant subspaces. Hence reducing a system of $N$ linear oscillator as Eq. (2) to a single linear mode, in order to predict the type of nonlinearity of the selected mode, may lead to erroneous results. The main reason comes from the non-invariance: as the trajectories computed with Eq. (58) do not exist for the complete system, there is 
no reason that the type of nonlinearity match. On the other hand, if one uses the NNMs, as invariance is recovered, the type of nonlinearity can be predicted, and for the same complexity at hand. Indeed, using the formalism presented previously, reducing the dynamics to the $p^{\text {th }}$ NNM leads to still consider a single oscillator equation, but inside wich all the non-resonant couplings have been included. The dynamics for a single NNM is given in Eq. (45). A first-order perturbative method then leads to the following relationship:

$$
\omega_{N L}=\omega_{0}\left(1+\Gamma_{p} a^{2}\right), \quad \text { with } \quad \Gamma_{p}=\frac{3\left(A_{p p p}^{p}+h_{p p p}^{p}\right)+\omega_{p}^{2} B_{p p p}^{p}}{8 \omega_{p}^{2}} .
$$

In this equation, on can see that the influence of all the slave linear modes (used to construct the corresponding NNM) are enclosed in the $A_{p p p}^{p}$ and $B_{p p p}^{p}$ coefficients. Their variation is thus key to properly predict the type of nonlinearity. From Eqs. (41), and substituting the expressions of $a_{p p}^{p}$ and $b_{p p}^{p}$ from the nonlinear transform (see Eqs.(107) in appendix A), $A_{p p p}^{p}$ and $B_{p p p}^{p}$ can be explicited as:

$$
\begin{aligned}
& A_{p p p}^{p}=\sum_{l=1}^{N} \frac{2 \omega_{p}^{2}-\omega_{l}^{2}}{\omega_{l}^{2}\left(\omega_{l}-2 \omega_{p}\right)\left(\omega_{l}+2 \omega_{p}\right)}\left(g_{p l}^{p}+g_{l p}^{p}\right) g_{p p}^{l}, \\
& B_{p p p}^{p}=\sum_{l=1}^{N} \frac{2}{\omega_{l}^{2}\left(\omega_{l}-2 \omega_{p}\right)\left(\omega_{l}+2 \omega_{p}\right)}\left(g_{p l}^{p}+g_{l p}^{p}\right) g_{p p}^{l} .
\end{aligned}
$$

Note that for applying these formulae, the implicit convention used throughout the notes: $g_{i j}^{p}=0$ when $i>j$, must be applied.

These expressions call for the following comments:

- Considering one single mode, say $p$, in the original equations of motion, would lead, by substitution, to recover the type of nonlinearity given in Eq. (59). The summations, in the case of a $N$-dofs system, clearly shows how all the slave modes can influence the type of nonlinearity.

- The expressions in Eqs (61) shows that in case of internal resonance, a small denominator effect appears, leading to a divergence in the expressions of $a_{p p}^{p}$ and $b_{p p}^{p}$, and thus on the predicted type of nonlinearity $\Gamma_{p}$. Even though this is in the line of all the calculations presented, a further comment is needed. Interestingly, there is only one kind of internal resonance, namely 2:1 resonance, which have an influence on the type of non-linearity. When studying the $p^{\text {th }}$ mode, only the $l^{\text {th }}$ modes, whose eigenfrequencies are such that $\omega_{l}=2 \omega_{p}$, are able to significantly change the value of $T_{p}$. Other second-order internal resonances, e.g. $\omega_{p}=2 \omega_{l}$, or $\omega_{l}+\omega_{m}=\omega_{p}$, are not able to produce a small denominator and to change the value of $T_{p}$. Finally, third-order internal resonances have no influence since only the first order correction to the backbone curve is studied (Eq. (60)).

- In case of 2:1 internal resonance, the system cannot be reduced to a single NNM. Morevover, perturbative studies shows that in the 2:1 internal resonance case, due to the presence of invariantbreaking terms, only coupled solutions exist. Thus the type of non-linearity, which is a notion associated to the backbone curve of a single oscillator, does not have anymore meaning.

The next subsection considers a two-dofs example in order to better highlight the main features of the method. Then continuous structures with an infinite number of dofs will be considered. The method of normal form for NNMs will there find a very good application, as it provides a reliable and efficient method to predict properly their type of nonlinearity.

\subsection{A two dofs example}

The two dofs system composed of a masse connnected to two nonlinear springs, whose equations of motions are given in Eqs. (42), is once again considered. 
Considering the first linear mode leads to a dynamics governed by:

$$
\ddot{X}_{1}+\omega_{1}^{2} X_{1}+\frac{3 \omega_{1}^{2}}{2} X_{1}^{2}+\frac{\omega_{1}^{2}+\omega_{2}^{2}}{2} X_{1}^{3}=0
$$

The backbone curve in this case reads:

$$
\tilde{\omega}_{N L}=\omega_{1}\left(1+\left(-\frac{3}{4}+\frac{3 \omega_{2}^{2}}{16 \omega_{1}^{2}}\right) a^{2}\right),
$$

where $\tilde{\omega}_{N L}$ stands for the non-linear angular frequency found with a linear mode approximation, and $a$ is the amplitude of the motion considered: $X_{1}=a \cos \left(\tilde{\omega}_{N L} t+\beta_{0}\right)+\ldots$

In the parameter plane $\left(\omega_{1}, \omega_{2}\right)$, regions of hardening or softening behaviour are governed by the sign of:

$$
\tilde{\Gamma}_{1}=-\frac{3}{4}+\frac{3 \omega_{2}^{2}}{16 \omega_{1}^{2}}
$$

Considering now the first non-linear mode, which is the right approximation if one is interested in a motion non-linearly vibrating along the first physical mode, indicates that the oscillations are governed by Eq. (45), with $p=1$. Computing the coefficients and replacing in Eq. (60) shows that the hardening or softening behaviour onto the first invariant manifold is determined by the sign of:

$$
\Gamma_{1}=-\frac{3}{4}+\frac{3 \omega_{2}^{2}}{16 \omega_{1}^{2}}+\frac{\omega_{2}^{2}\left(8 \omega_{1}^{2}-3 \omega_{2}^{2}\right)}{16 \omega_{1}^{2}\left(\omega_{2}^{2}-4 \omega_{1}^{2}\right)}
$$
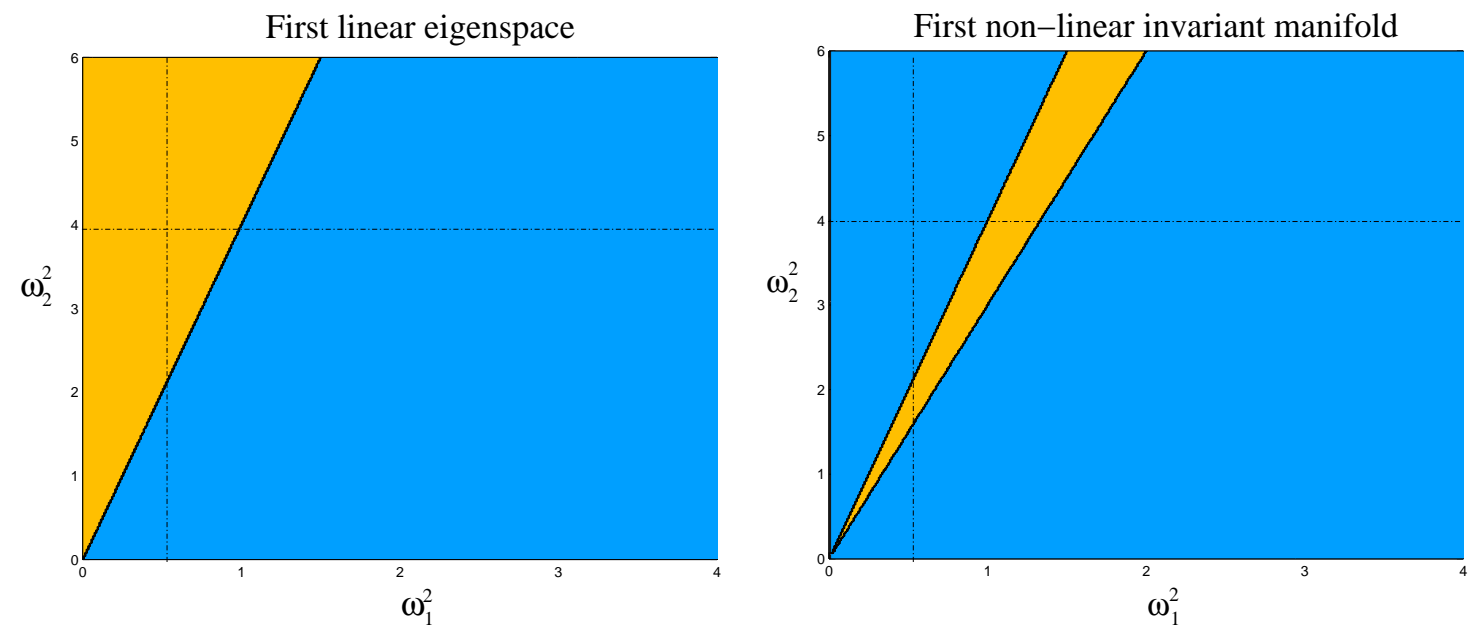

Figure 10: Regions of hardening/softening behaviour in the parameter plane $\left(\omega_{1}^{2}, \omega_{2}^{2}\right)$, for the two-dofs system. Yellow: hardening behaviour, blue: softening behaviour. Left: prediction given by the sign of $\tilde{\Gamma}_{1}$, i.e. with truncation to the first linear mode. Right: prediction given by the sign of $\Gamma_{1}$, i.e. for oscillatory motions along the first NNM.

The behaviour of $\tilde{\Gamma}_{1}$ shows that a hardening behaviour is found when $\omega_{2}$ is large as compared to $\omega_{1}$, otherwise a softening behaviour is at hand. This can be easily understood by comparing the quadratic and cubic coefficients of the oscillator-equation (62). On the other hand, the prediction for hardening/softening region in parameter space with a single NNM truncation shows clearly the correction brought by considering properly the bending of the phase space caused by the presence of the second oscillator. More specifically, this effect can be drastic and can change the effective behaviour of the 
non-linear oscillations. Figure 10 shows the hardening and softening region in the two cases (simply given by the signs of $\tilde{\Gamma}_{1}$ in the linear case, and $\Gamma_{1}$ in the non-linear case). One can notice for example the upper-left region, which is predicted to exhibit a hardening behaviour by the linear approximation, whereas the real behaviour is soft.

In order to have a better picture of the behaviour of the type of non linearity versus the two parameters $\left(\omega_{1}, \omega_{2}\right)$ of the system, Figure 11 shows two cuts in this two-dimensional parameter plane, namely for fixed $\omega_{2}=2$ and $\omega_{1}$ variable, then for fixed $\omega_{1}=\sqrt{0.5}$ and $\omega_{2}$ variable, i.e. along the two lines indicated in Fig. 10. These cuts reveals that the behaviour of $\tilde{\Gamma}_{1}$ is monotone, whereas $\Gamma_{1}$ shows a singularity, occuring for $\omega_{2}=2 \omega_{1}$, i.e. at the 2:1 internal resonance between the two eigenfrequencies. As already underlined, in the vicinity of this internal resonance, the concept of the type of nonlinearity loses its meaning because the dynamics is essentially two-dimensional and cannot be reduced to a single NNM. However, far from the 2:1 resonance, the predicted type of nonlinearity from the single NNM solution is reliable, and depart from the approximation given by considering a single linear mode.

(a)

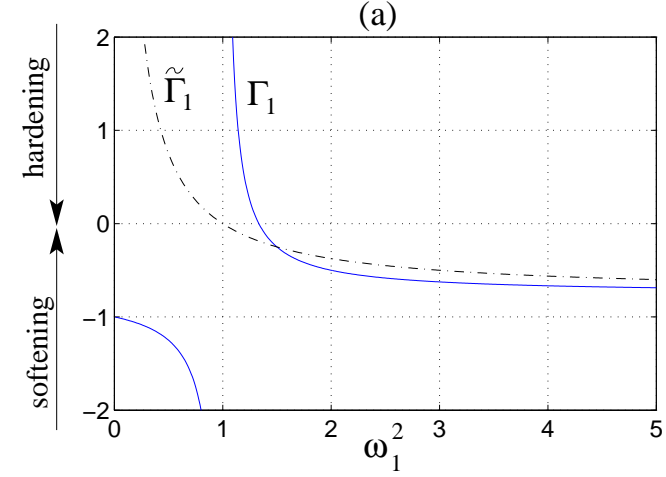

(b)

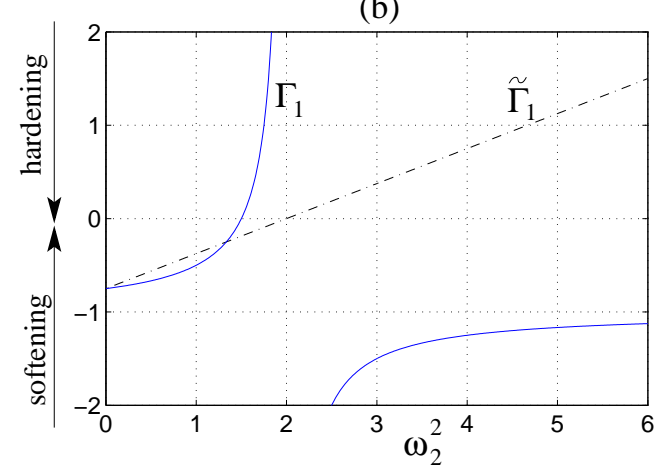

Figure 11: Type of nonlinearity for the two-dofs example, comparison between the prediction given by a single linear mode, $\tilde{\Gamma}_{1}$, versus the correct prediction given by a single NNM, $\Gamma_{1}$. (a): $\omega_{2}=2, \omega_{1}^{2} \in[0,5]$, (a): $\omega_{1}=\sqrt{0.5}, \omega_{2}^{2} \in[0,5]$

\subsection{Application to shells}

The method shown previously for predicting accurately the type of nonlinearity, is now applied to the case of spherical-cap thin shallow shells with a varying radius of curvature $R$. Flat plates are known to exhibit a hardening behaviour,as it has been shown both theoretically and experimentally (see e.g. $[48,58,35,44,54,46])$, which means that the leading cubic coefficient $h_{p p p}^{p}$ is positive. Introducing a radius of curvature $R$, going to infinity (perfect plate) to finite values (spherical-cap shells) introduces an asymmetry in the restoring force, due to the loss of symmetry of the neutral plane of the shell. In turn, quadratic nonlinearity appears in the equations of motions, with a magnitude proportioanl to $1 / R$. The type of nonlinearity is thus awaited to vary from hardening to softening type, depending on the selected mode and the geometry. The aim of this subsection is to derive properly the prediction of this type of nonlinearity for spherical shells, with the formalism of NNMs and normal form.

The model is based on von Kármán kinematical assumptions on the strain-displacement relationship, in order to take into account moderately large vibration amplitudes. The governing equations are first recalled, then the Galerkin method is briefly reviewed in order to explain how to pass from the PDE of motion to oscillator-equations having the form of Eqs. (2), then finally the type of nonlinearity for some eigenmodes of the structure, are given. A geometrical nondimensional parameter, inversely proportional to the curvature of the shell, is introduced, in order to study how the type of nonlinearity is modified when continuously transforming a thin circular plate to a spherical-cap shell. 


\subsubsection{Von Kármán model}

Von Kármán kinematical assumption relies in a clever simplification of the general 3-D strain-displacement relationship, allowing to take into account moderately large vibration amplitudes, where the coupling between in-plane and transverse motions is taken into account. The model has first been written for the static behaviour of plates [25], and has then been generalized to dynamical behaviour of plates and shells $[11,14]$. In this subsection we follow the derivations proposed in [53]. A spherical shell of thickness $h$, radius of curvature $R$ and outer diameter $2 a$, made of a homogeneous isotropic material of density $\rho$, Poisson ratio $\nu$ and Young's modulus $E$, see Fig. 12. Numerous assumptions pertaining to the derivation of von Kármán model - e.g. moderate rotations, in-plane inertia - are not recalled here for the sake of brevity, the interested reader is referred to $[11,14,54,47,45]$ for a more thorough description. About the geometry of the shell, it is assumed that:

- the shell is thin: $h / a \ll 1$ and $h / R \ll 1$;

- the shell is shallow: $a / R \ll 1$;

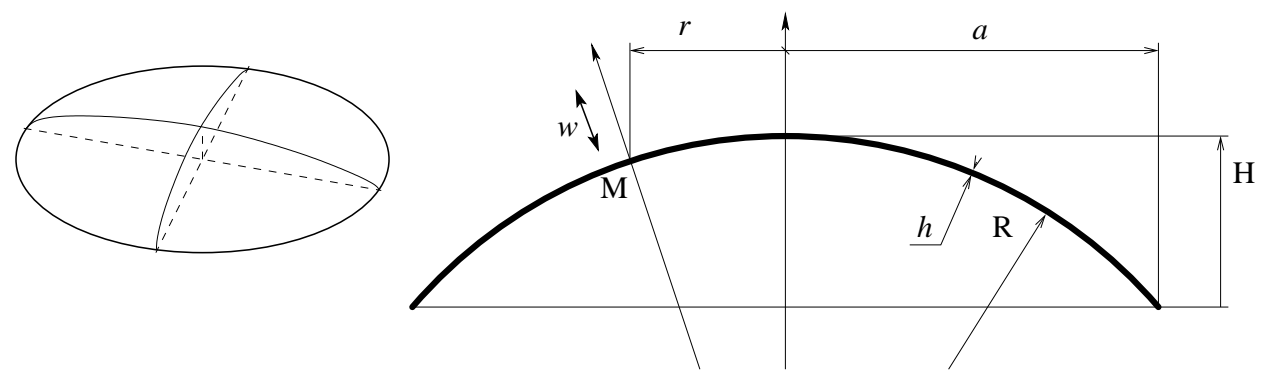

Figure 12: Geometry of the shell: three-dimensional sketch and cross section.

The equations of motion are given in terms of the transverse displacement $w(r, \theta, t)$ along the normal to the mid-surface and the Airy stress function $F(r, \theta, t)$. This is a peculiarity of the von Kármán model, which allows expression of the in-plane motions into the so-called Airy stress function $F$. The equations of motion reads, for all time $t$ :

$$
\begin{aligned}
D \Delta \Delta w+\frac{1}{R} \Delta F+\rho h \ddot{w} & =L(w, F), \\
\Delta \Delta F-\frac{E h}{R} \Delta w & =-\frac{E h}{2} L(w, w),
\end{aligned}
$$

where $D=E h^{3} / 12\left(1-\nu^{2}\right)$ is the flexural rigidity, $\ddot{w}$ is the second partial derivative of $w$ with respect to time, $\Delta$ is the laplacian and $L$ is a bilinear quadratic operator. With the assumption of a shallow shell fulfilled, the spatial operators are written in polar coordinates, and thus reads:

$$
\Delta(\cdot)=(\cdot)_{, r r}+\frac{1}{r}(\cdot)_{, r}+\frac{1}{r^{2}}(\cdot)_{, \theta \theta},
$$

and

$$
L(w, F)=w_{, r r}\left(\frac{F_{, r}}{r}+\frac{F_{, \theta \theta}}{r^{2}}\right)+F_{, r r}\left(\frac{w_{, r}}{r}+\frac{w_{, \theta \theta}}{r^{2}}\right)-2\left(\frac{w_{, r \theta}}{r}-\frac{w_{, \theta}}{r^{2}}\right)\left(\frac{F_{, r \theta}}{r}-\frac{F_{, \theta}}{r^{2}}\right),
$$

Eqs (66) express the dynamics of a spherical-cap shell, without external forcing, nor damping terms. These equations are valid for plates as well, which is obtained for a radius of curvature $R$ going to infinity. This, in turn, simplifies two terms in (66). The linear remaining terms in Eq. (66a) are the 
classical inertia and flexural rigidity, that one finds back in the linear Kirchhoff-Love model for vibration of plates. The linear coupling term between Eqs (66a) and (66b), proportional to $1 / R$, expresses the linear coupling between transverse and in-plane motions, which exist for shells only. For plates, these terms vanish. Finally, the nonlinear coupling term reflects the nonlinear coupling between transverse and in-plane motions. One can observe that for shells, this coupling implies quadratic and cubic terms for the displacement $w$, whereas for plates, only cubic terms are present.

Dimensionless variables are introduced as:

$$
r=a \bar{r}, \quad t=a^{2} \sqrt{\rho h / D} \bar{t}, \quad w=h \bar{w}, \quad F=E h^{3} \bar{F}
$$

Substituting the above definitions in equations of motion (66a,b) and dropping the overbars in the results, one obtains:

$$
\begin{aligned}
& \Delta \Delta w+\varepsilon_{q} \Delta F+\ddot{w}=\varepsilon_{c} L(w, F), \\
& \Delta \Delta F-\sqrt{\kappa} \Delta w=-\frac{1}{2} L(w, w),
\end{aligned}
$$

where the aspect ratio $\kappa$ of the shell has been introduced:

$$
\kappa=\frac{a^{4}}{R^{2} h^{2}}
$$

As it will be shown next, for a fixed value of the Poisson ratio $\nu$, all the linear results (eigenfrequencies and mode shapes), as well as the type of non-linearity, only depend on $\kappa$, which is the only free parameter related to the geometry of the shell. The two other parameters $\varepsilon_{q}$ and $\varepsilon_{c}$ appearing in Eq. (70) are equal to:

$$
\varepsilon_{q}=12\left(1-\nu^{2}\right) \frac{a^{2}}{R h}=12\left(1-\nu^{2}\right) \sqrt{\kappa}, \quad \varepsilon_{c}=12\left(1-\nu^{2}\right) .
$$

Their subscripts comes from the fact that they balance respectively the quadratic and the cubic terms in the non-linear ordinary differential equations governing the dynamics of the problem (see Eq. (75)).

\subsubsection{Linear analysis}

All the analysis is here performed for a free-edge boundary condition. It is derived by vanishing, at the edge $r=1$ : the membrane forces, the bending moment, the twisting moment and the transverse shear force. They are not recalled here for the sake of brevity, the interested reader can found the complete expressions in $[47,53]$.

The linearized equations of motion are analyzed to derive the eigenmodes and eigenfrequencies of the problem, as a function of the geometry. The eigenmodes are the solutions of:

$$
\begin{aligned}
& \Delta \Delta \Phi+\chi \Delta \Psi-\omega^{2} \Phi=0, \\
& \Delta \Delta \Psi=\Delta \Phi .
\end{aligned}
$$

where $\Phi$ refers to the eigenmodes of the transverse motion and $\Psi$ to those of the membrane motion. The coefficient $\chi=12\left(1-\nu^{2}\right) \kappa$ is the only parameter of the linear problem. All the study could have been realized by taking $\chi$ as the geometrical parameter, as it is sometimes done by various authors $[16,18]$. However, the results will be presented as functions of $\kappa$, in order to set apart the material property which appear through the Poisson ratio $\nu$ in the expression of $\chi$. In the remainder of this study, $\nu$ is kept constant at $\nu=0.33$.

Transverse and membrane mode shapes are numbered $\Phi_{(k, n)}$ and $\Psi_{(k, n)}$ where $k$ is the number of nodal diameters and $n$ the number of nodal circles. Axisymmetric modes are such that $k=0$. For $k \geq 1$ 

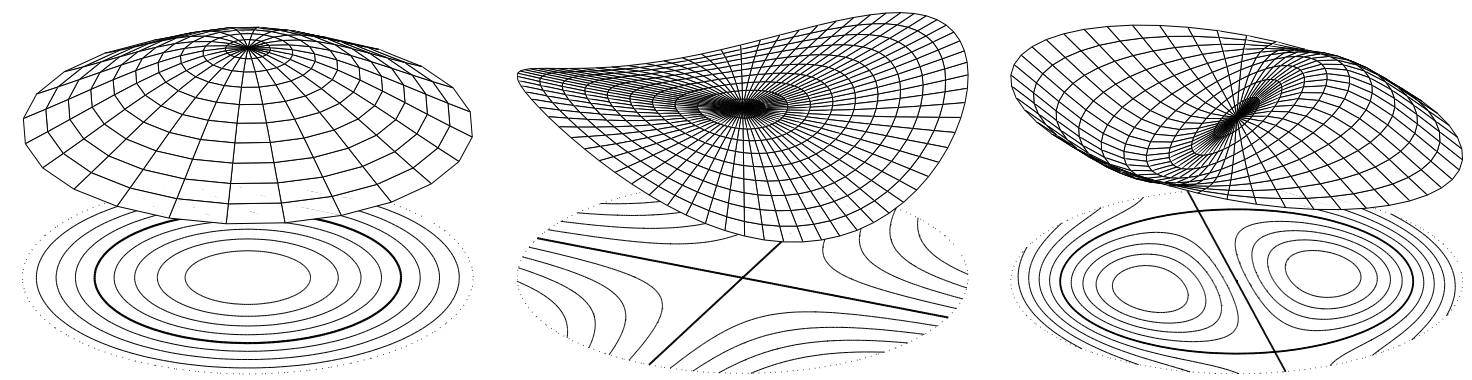

Figure 13: Three representative mode shapes of a circular spherical thin shallow shell with free edge: axisymmetric mode $(0,1)$, purely asymmetric mode $(2,0)$ and mixed mode $(1,1)$.

(asymmetric modes), the associated eigenvalue has a multiplicity of two, so that for each eigenfrequency, there are two independent mode shapes, called preferential configurations or companion modes. Among these modes, purely asymmetric modes (such that $k \geq 2$ and $n=0$ ) are distinguished from mixed modes (such that $k \geq 1$ and $n \geq 1$ ). Figure 13 shows representative pictures for each of the three distinguished family. Mode $(0,1)$ is axisymmetric, mode $(2,0)$ is a purely asymmetric one while $(1,1)$ is a mixed mode.

The complete linear analysis is provided in [47]. It shows that all deformed shapes, except membrane mode shapes for purely asymmetric modes, have a negligible dependence on the geometrical parameter $\kappa$. This is illustrated in Fig. 14, showing the profile for $r \in[0,1]$ of two different modes: $\Phi_{(2,0)}$, the first purely asymmetric transverse mode, and $\Psi_{(2,0)}$, the first purely asymmetric in-plane mode, for large variations of the aspect ratio $a / R$ between 0 and 0.6 (remind that the shallowness asummption implies $a / R \ll 1)$. The dependence of $\Phi_{(2,0)}$ with the geometry is very slight, and this kind of behaviour is also found for all transverse modes, all in-plane modes except purely asymmetric ones.
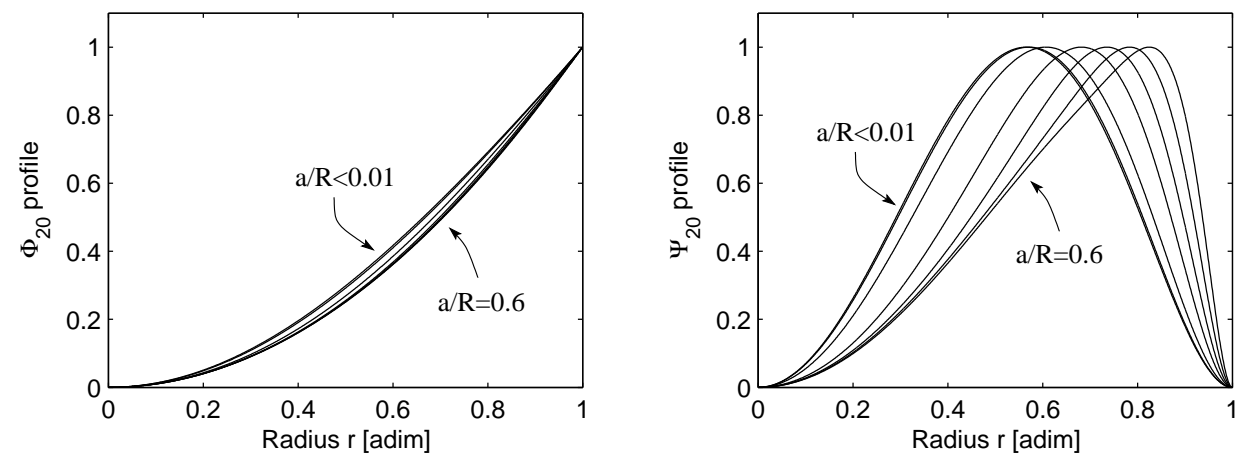

Figure 14: Profiles of theoretical asymmetric $(2,0)$ mode shape, for several values of a/R between 0 and 0.6: (left) transverse mode and (right) membrane mode.

On the contrary, the eigenfrequencies dependence on the aspect ratio $\kappa$, represented on Fig. 15, shows a different behaviour, which leads to classify the modes into two families. The first family contains the purely asymmetric modes, since their eigenfrequencies display a slight dependence on curvature. The second family contains axisymmetric and mixed modes. They show a huge eigenfrequency dependence on curvature. 


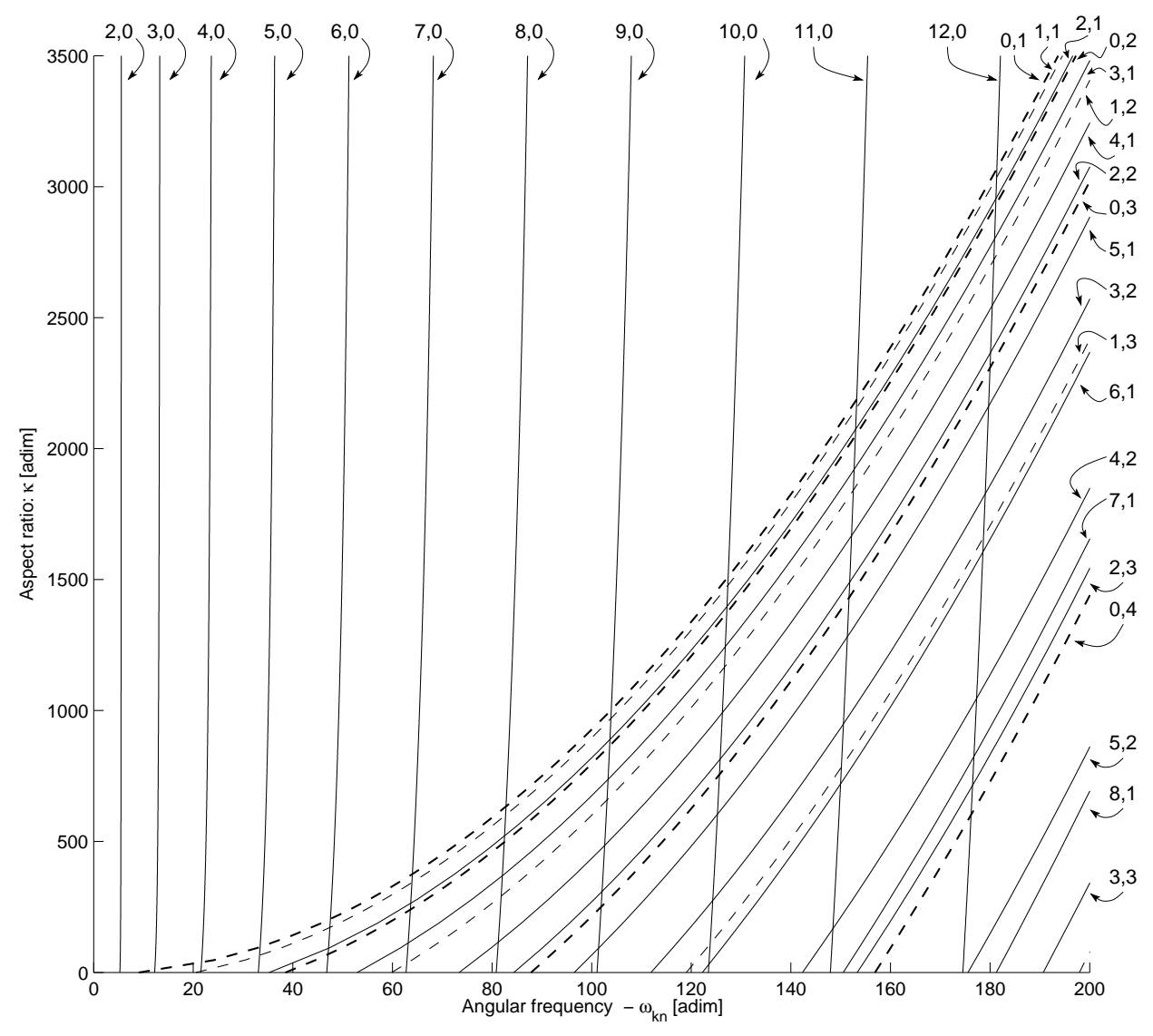

Figure 15: Dimensionless natural frequencies $\omega_{k n}$ of the shell as a function of the aspect ratio $\kappa$, for $\nu=0.33$.

\subsubsection{Modal expansion}

The complete non-linear equations of motion (70) are projected onto the natural basis of the transverse eigenmodes. The displacement is thus written as:

$$
w(r, \theta, t)=\sum_{p=1}^{+\infty} X_{p}(t) \Phi_{p}(r, \theta),
$$

where the subscript $p$ refers to a specific mode of the shell, defined by a couple $(k, n)$ and, if $k \neq 0$, an additional binary variable which indicates the preferential configuration considered (sine or cosine companion mode). The modal displacements $X_{p}$ are the unknowns, and their dynamics is governed by: $\forall p \geq 1$ :

$$
\ddot{X}_{p}+\omega_{p}^{2} X_{p}+\varepsilon_{q} \sum_{i=1}^{+\infty} \sum_{j=1}^{+\infty} g_{i j}^{p} X_{i} X_{j}+\varepsilon_{c} \sum_{i=1}^{+\infty} \sum_{j=1}^{+\infty} \sum_{k=1}^{+\infty} h_{i j k}^{p} X_{i} X_{j} X_{k}=0 .
$$

The expression of the non-linear coefficients are:

$$
\begin{aligned}
& g_{i j}^{p}=-\iint_{\mathcal{S}_{\perp}} \Phi_{p} L\left(\Phi_{i}, \Psi_{j}\right) d S-\frac{1}{2} \sum_{b=1}^{+\infty} \frac{1}{\xi_{b}^{4}} \iint_{\mathcal{S}_{\perp}} L\left(\Phi_{i}, \Phi_{j}\right) \Upsilon_{b} d S \iint_{\mathcal{S}_{\perp}} \Phi_{p} \Delta \Upsilon_{b} d S \\
& h_{i j k}^{p}=\frac{1}{2} \sum_{b=1}^{+\infty} \frac{1}{\xi_{b}^{4}} \iint_{\mathcal{S}_{\perp}} L\left(\Phi_{i}, \Phi_{j}\right) \Upsilon_{b} d S \iint_{\mathcal{S}_{\perp}} \Phi_{p} L\left(\Phi_{k}, \Upsilon_{b}\right) d S
\end{aligned}
$$


The $\Upsilon_{n}$, as well as its associated zero $\xi_{n}$, are defined in [47]. $\mathcal{S}_{\perp}$ is the domain defined by $(r, \theta) \in$ $\left[\begin{array}{ll}0 & 1]\end{array} \times\left[\begin{array}{ll}0 & 2 \pi[\text {. }\end{array}\right.\right.$

Now that the PDE has been reduced to nonlinear oscillator equations, the formalism of NNMs and normal form can be applied to derive the type of nonlinearity for each mode of the shell. The type of nonlinearity is dictated by the sign of:

$$
T_{p}=\frac{1}{8 \omega_{p}^{2}}\left[3\left(A_{p p p}^{p}+\varepsilon_{c} h_{p p p}^{p}\right)+\omega_{p}^{2} B_{p p p}^{p}\right],
$$

where the expressions for $A_{p p p}^{p}$ and $B_{p p p}^{p}$ are given as in Eq. (61):

$$
\begin{aligned}
& A_{p p p}^{p}=\varepsilon_{q}^{2} \sum_{l=1}^{+\infty} \frac{2 \omega_{p}^{2}-\omega_{l}^{2}}{\omega_{l}^{2}\left(\omega_{l}^{2}-4 \omega_{p}^{2}\right)}\left(g_{p l}^{p}+g_{l p}^{p}\right) g_{p p}^{l}, \\
& B_{p p p}^{p}=\varepsilon_{q}^{2} \sum_{l=1}^{+\infty} \frac{2}{\omega_{l}^{2}\left(\omega_{l}^{2}-4 \omega_{p}^{2}\right)}\left(g_{p l}^{p}+g_{l p}^{p}\right) g_{p p}^{l} .
\end{aligned}
$$

Before showing the results, two important comments are worth mentionable:

- The nonlinear coefficients $g_{i j}^{p}$ and $h_{i j k}^{p}$ shows a very slight dependence on the curvature of the shell. This is the consequence of the slight dependence of the mode shapes with the aspect ratio, as the non-linear coefficients are computed from integrals involving the mode shape functions (Eqs (76-77)). Hence the main effect of the shell's geometry on the trend of non-linearity is described by the relative variations of the eigenfrequencies, shown on Fig. 15.

- As it appears in Eqs (79-80), when studying the trend of non-linearity of the $p^{\text {th }}$ mode, one has to keep all the $l$ modes such that $g_{p p}^{l} \neq 0$, and $g_{p l}^{p} \neq 0$ or $g_{l p}^{p} \neq 0$. As shown in [47], a number of coefficients $\left\{g_{i j}^{p}\right\}_{p, i, j \geq 1}$ are equal to zero due to the rotational symmetry of the structure. The conditions for these quadratic coefficients to be non-zero are expressed in terms of the number of nodal diameters $k_{l}$ and $k_{p}$ of the $l$ and $p$ modes. They read:

(i) $g_{p p}^{l} \neq 0$ if $k_{l} \in\left\{2 k_{p}, 0\right\}$.

(ii) $g_{p l}^{p} \neq 0$ or $g_{l p}^{p} \neq 0$ if $k_{p} \in\left\{k_{l}+k_{p},\left|k_{l}-k_{p}\right|\right\}$.

These rules show that two classes of modes have to be retained when studying the type of nonlinearity of the $p^{\text {th }}$ mode: axisymmetric $\left(k_{l}=0\right)$ as well as asymmetric modes having twice the number of nodal diameters $\left(k_{l}=2 k_{p}\right)$. No other mode has an influence on the type of non-linearity.

In the remainder of the study, $N$ will refer to the number of modes retained in this specific subset composed of the pertinent ones with respect to the type of non-linearity.

\subsubsection{From circular plates to spherical-cap shells}

The type of nonlinearity is now computed for three different modes of the shell, representing each of the three families. A purely asymmetric mode, $(4,0)$, is first selected. The variation of $T_{(4,0)}$ as function of $\kappa$ is shown in Fig. 16. According to the rules underlined for truncation, only axisymmetric modes and asymmetric modes with eight nodal diameters, must be taken into account. For $\kappa=0$, the shell is a perfect circular plate. In this case the leading cubic coefficient is positive, so that a hardening behaviour is at hand. The dashed line $(N=1)$ shows the prediction given by considering only the linear mode for computing the type of nonlinearity. The correct prediction is severely affected by the presence of 2:1 internal resonances, creating discontinuities. For mode $(4,0)$, only two $2: 1$ internal resonances are possible with the modes that could interact to influence the type of nonlinearity: with mode $(0,2)$ at 


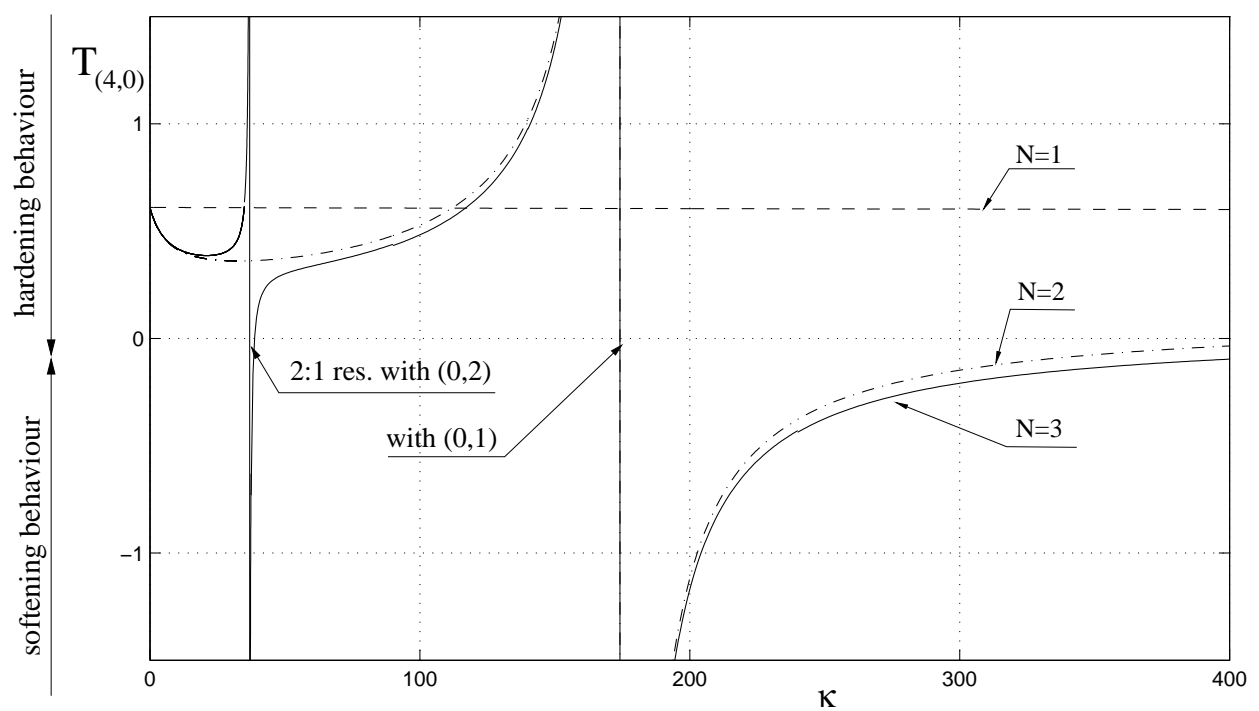

Figure 16: Type of nonlinearity for mode $(4,0)$ as function of the geometrical parameter $\kappa . \mathrm{N}=1$ : system truncate to linear mode $(4,0) . \mathrm{N}=2$ : truncation including $(4,0)$ and $(0,1) . \mathrm{N}=3:(4,0),(0,1)$ and $(0,2)$.

$\kappa=36.91$, and with mode $(0,1)$ at $\kappa=174.1$. In conclusion for this purely asymmetric mode, one can underline the fundamental importance of axisymmetric modes for an accurate prediction of the type of nonlinearity. Secondly, hardening behaviour is observed until the 2:1 resonance with mode $(0,1)$, where softening behaviour settles down. Finally, The type of non-linearity tends to zero as $\kappa$ tends to infinity.

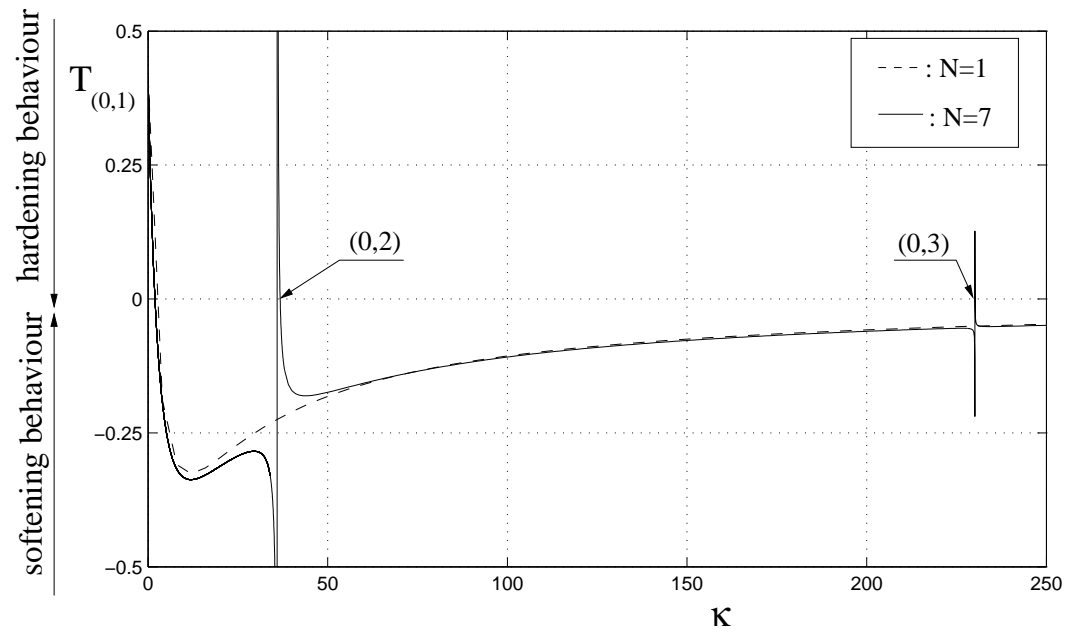

Figure 17: Type of nonlinearity for mode $(0,1)$ as function of the geometrical parameter $\kappa . \mathrm{N}=1$ : truncation containing only mode $(0,1) . \mathrm{N}=7$ : converged result with inclusion of the seventh first axisymmetric modes, from $(0,1)$ to $(0,7)$.

The case of an axisymmetric mode is now considered with mode $(0,1)$. The coupling rules (i) and (ii), indicate that only axisymmetric modes have to be kept. The main difference with the previous case is the behaviour of the eigenfrequencies with respect to $\kappa$. As it can be seen on Fig. 15, axisymmetric eigenfrequencies increase with curvature. Hence, an infinity of 2:1 internal resonances are now possible, with all the other axisymmetric modes.

The result of computation is shown on Fig. 17. It can be seen that the effect of the geometry -the 
increase of $\kappa$ - is much more pronounced than for the asymmetric mode: the inital hardening behaviour $(\kappa=0)$ becomes softening at $\kappa=1.93$, and not because of a 2:1 internal resonance. Two resonances, leading to a change in behaviour, are then shown: at $\kappa=35.97$, where the following relationship is fulfilled: $2 \omega_{(0,1)}=\omega_{(0,2)}=43.21$. Then at $\kappa=230.1$, where $2: 1$ resonance occurs with mode $(0,3)$. These 2:1 resonances lead to a return to hardening behaviour. However, it occurs on a very little interval, which is already negligible for the resonance with $(0,2)$, and completely negligible for $(0,3)$. The next 2:1 resonances (with $(0,4)$ at $\kappa=756.9$, with $(0,5)$ at $\kappa=1871.5 \ldots$ ) occur on intervals which are always smaller and thus are not shown.

Single-mode prediction is also shown on Fig. 17. Although the 2:1 resonances are missed, the general behaviour is correctly predicted: change from hardening to softening due to curvature is found at $\kappa=$ 1.95 instead of $\kappa=1.93$, and the asymptotic behaviour, which becomes neutral when $\kappa$ tends to infinity, is recovered. These results show that for the specific case of the fundamental axisymmetric mode, the single-mode approximation, used in the precedent studies $[19,59,41,56]$, predicts the essential features, in spite of a too severe truncation.

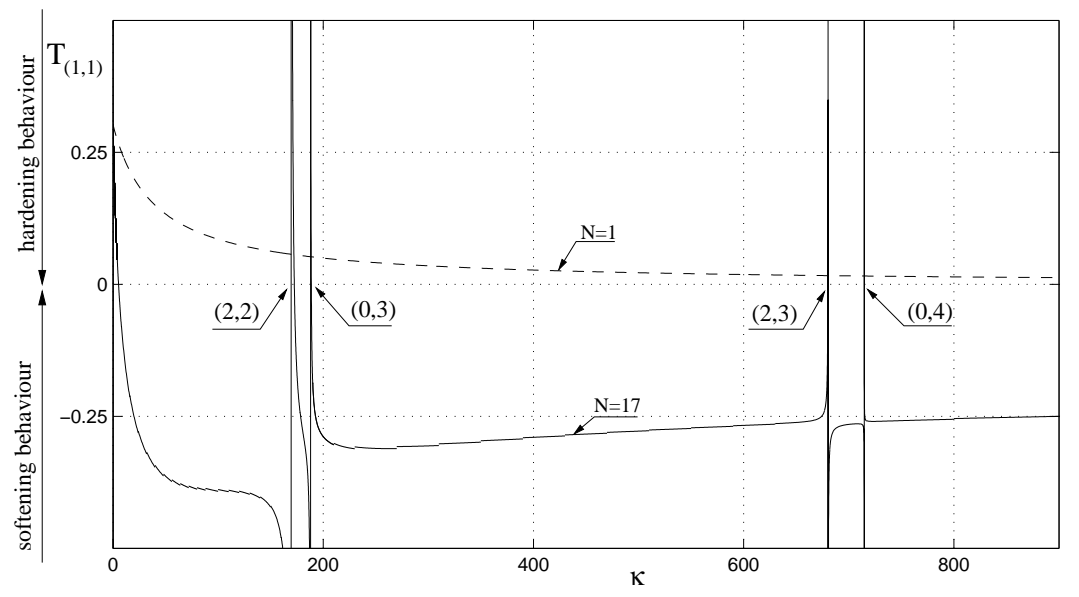

Figure 18: Type of nonlinearity for mode $(1,1)$ as function of the geometrical parameter $\kappa . \mathrm{N}=1$ : truncation including only linear mode $(1,1)$. $N=17$ : converged result including modes $(1,1),(2,0)$ to $(2,4)$ (with both configurations), $(0,1)$ to $(0,5)$.

The case of a mixed mode, namely $(1,1)$ is presented on Fig. 18. As for the axisymmetric modes, the effect of geometry is important and leads to a change of behaviour for a very small value of the aspect ratio: $\kappa=5.3$. Then $2: 1$ internal resonances occurs, with modes $(2,2),(0,3),(2,3),(0,4), \ldots$ Their number is unlimited, as for the axisymmetric case. The change of behaviour occurs on very small intervals. The first one, due to $2: 1$ resonance with mode $(2,2)$, is hardly negligible, and the others have no chance to be experimentally measurable. It can be thus conclude that except on a very small interval $(\kappa \in[0,5.3])$, mode $(1,1)$ behaves in a softening way.

The single-mode approximation is also shown on Fig. 18. It predicts a hardening behaviour which becomes neutral when $\kappa$ tends to infinity. The converged result is obtained for $N=17$ modes, namely : $(1,1) ;(2,0)$ to $(2,4) ;(0,1)$ to $(0,5)$, and shows that, contrary to the precedent cases, coefficient $T_{(1,1)}$ tends to a finite value when $\kappa$ tends to infinity. Hence the behaviour remains softening and does not becomes neutral for large values of the aspect ratio.

\subsection{Influence of the damping}

This subsection is devoted to studying the influence of the damping on the type of nonlinearity. This problematic could appear unusual or ill-posed, because the type of nonlinearity is generally defined for 
conservative systems through the backbone curve. However, a more general view is needed to tackle the cases of forced responses and/or dissipative free oscillations, where the oscillation frequency also depend on vibration amplitude.

As it has been shown in subsection 2.8, the normal form theory with inclusion of damping allows extending the results continuously from conservative to dissipative systems. Moreover, a clear dependence of the coefficients on the damping ratios, has been underlined. The aim of this subsection is thus to show how this dependence can influence the type of nonlinearity.

For that purpose, let us consider the two-dofs system, Eqs. (42), with inclusion of damping terms. The motions on the first NNM are governed by the dynamical reduced equation:

$$
\ddot{R}_{1}+\omega_{1}^{2} R_{1}+2 \xi_{1} \omega_{1} \dot{R}_{1}+\left(h_{111}^{1}+A_{111}^{1}\right) R_{1}^{3}+B_{111}^{1} R_{1} \dot{R}_{1}^{2}+C_{111}^{1} R_{1}^{2} \dot{R}_{1}=0 .
$$

and the type of nonlinearity is given by the sign of $\Gamma_{1}$ :

$$
\Gamma_{1}=\frac{3\left(A_{111}^{1}+h_{111}^{1}\right)+\omega_{1}^{2} B_{111}^{1}}{8 \omega_{1}^{2}}
$$

As a consequence of the particular behaviour of the $A_{111}^{1}$ and $B_{111}^{1}$ with increasing values of $\xi_{2}$, the type of non-linearity may change with increasing damping. Figure 19 shows, for the two-dofs example with parameter values $\omega_{1}=3, \omega_{2}=5.4$, and $\xi_{1}=0.001$, that when $\xi_{2}$ increases (simulating the presence of a slave mode which is more and more damped) the type of non-linearity of the first mode may be affected and change from hardening to softening behaviour. In this case, it happens for $\xi_{2}=0.081$, so that the ratio of the two modal damping is equal to: $\xi_{2} / \xi_{1}=81$.

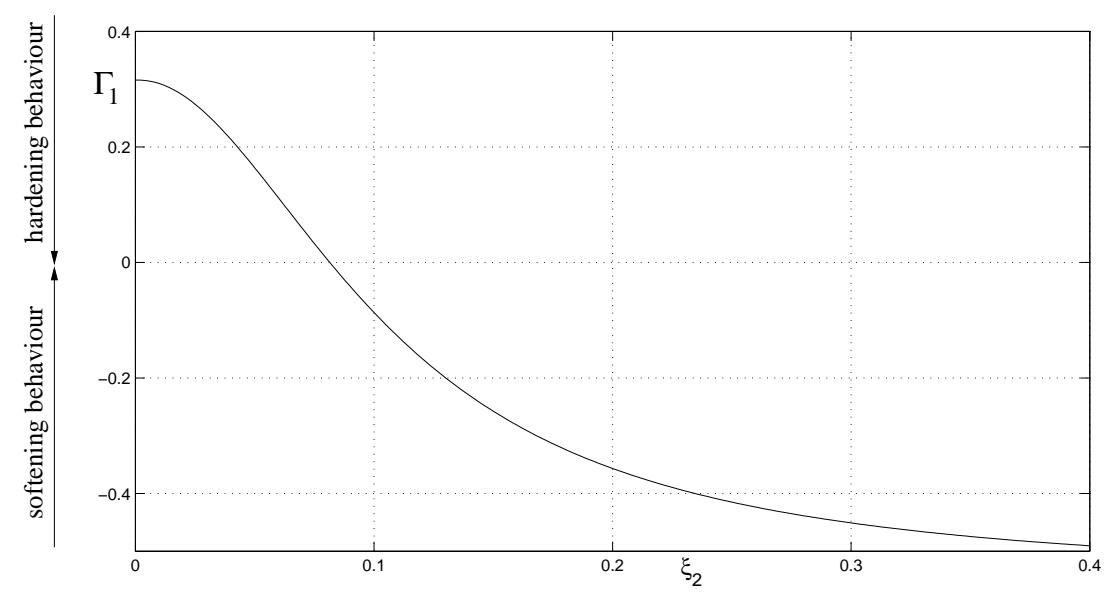

Figure 19: Type of non-linearity $\Gamma_{1}$, defined by Eq. (82), for increasing values of $\xi_{2}$. The behaviour turns from hardening to softening type for $\xi_{2}=0.081$. Other selected values are: $\omega_{1}=3, \omega_{2}=5.4$, and $\xi_{1}=0.001$.

Another case is studied in Fig. 20, where now the two linear modal damping coefficients $\xi_{1}$ and $\xi_{2}$ vary of the same quantity, so that the ratio $\xi_{2} / \xi_{1}$ is kept constant. This more realistic case could for example simulate a structure whose global damping is raised. Here again, it is also observed that a global increase of the amount of damping have a significant effect on the type of non-linearity. From these two examples, it is concluded that the damping tends to enhance and favours the softening behaviour.

This particular effect of the damping on the type of non-linearity can thus significantly change predictions based on the undamped system. Let us now observe how the type of nonlinearity is modified on a section of the map of nonlinearity as function of the two parameters $\left(\omega_{1}, \omega_{2}\right)$, shown in Fig. 10. More precisely, the line $\omega_{2}=2$ and varying $\omega_{1}$, already shown in Fig. 11(a), is now considered, for a fixed value of $\xi_{1}=0.001$, and increasing values of $\xi_{2}$. The results are given in Fig. 21 . 


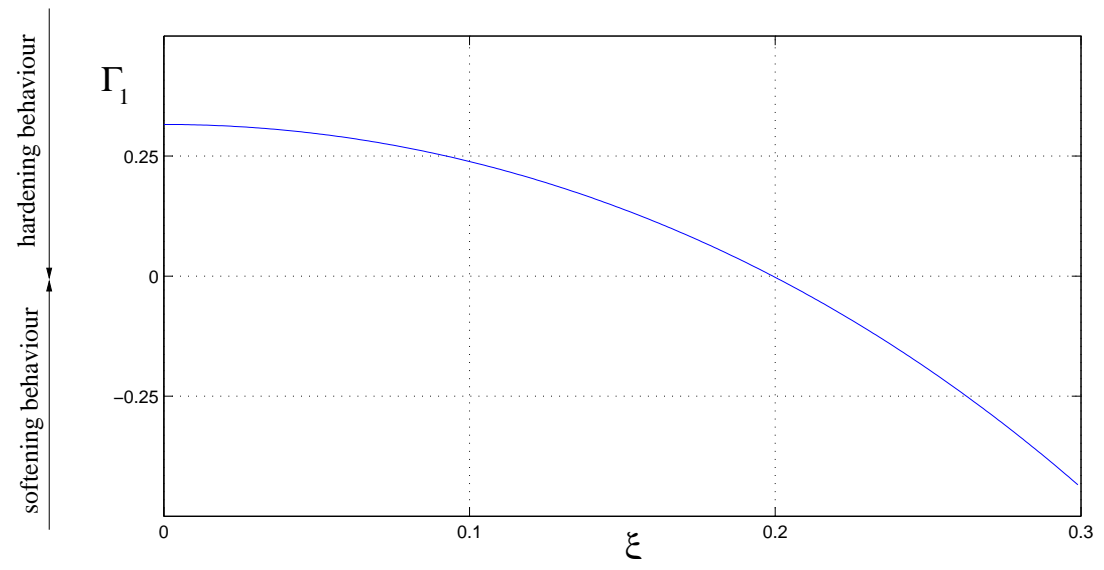

Figure 20: Type of non-linearity $\Gamma_{1}$ for increasing values of the global damping in the system. The two modal damping values here are equal $: \xi_{1}=\xi_{2}=\xi$. Other selected values are: $\omega_{1}=3, \omega_{2}=5.4$.

When damping is not considered, the type of non-linearity $\Gamma_{1}$ displays a discontinuity at the internal resonance value where $\omega_{1}=1$, ie. where $2: 1$ resonance occurs : $\omega_{2}=2 \omega_{1}$. At this discontinuity point, the behaviour changes abruptly from softening to hardening type. The discontinuity is due to the presence of internal resonance which leads to small denominators in the solution. As already argued, in a small intervall near the 2:1 internal resonance point, single-mode solutions do not exist anymore, and the concept of the type of non-linearity loses its meaning.

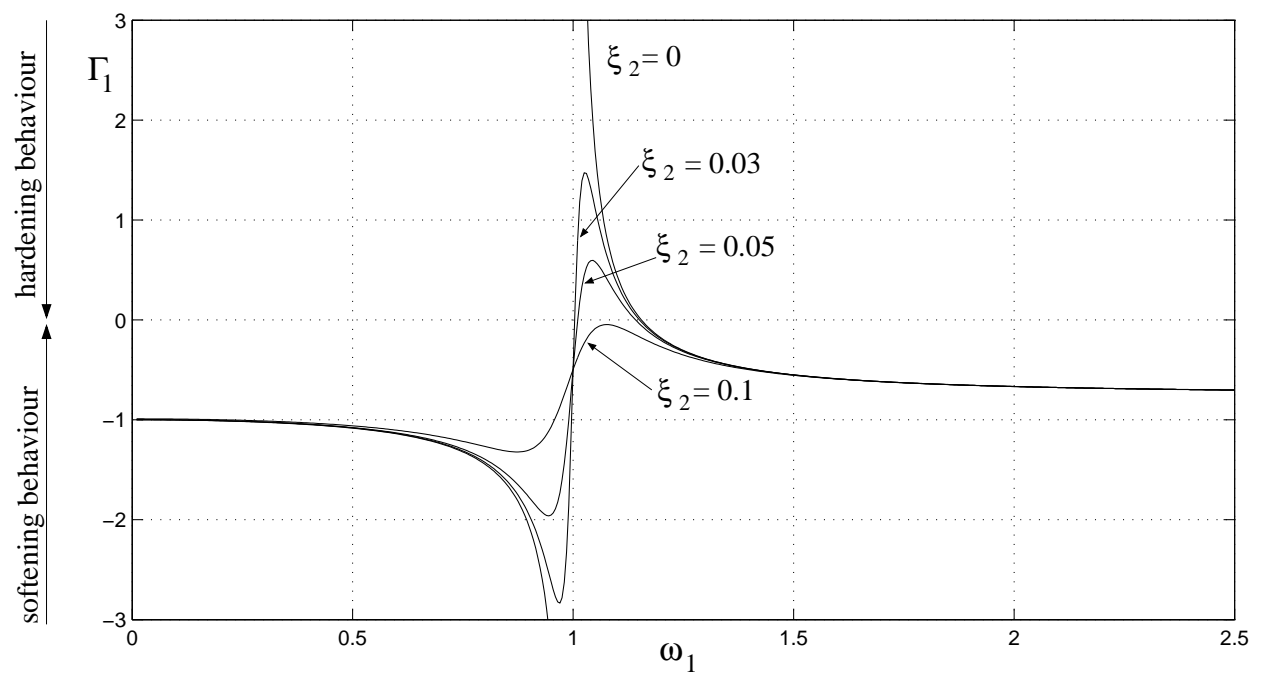

Figure 21: Type of non-linearity for different values of $\xi_{2}$, illustrating the fact that the discontinuity at the 2:1 internal resonance is smoothened by increasing the damping of the slave oscillator. $\omega_{2}=2$ and $\xi_{1}=0.001$.

Here, it is shown that taking into account the whole damping of the structure smoothens the discontinuity. For increasing values of $\xi_{2}$, Figure 21 shows that the region of hardening behaviour after the 2:1 internal resonance decreases, and can even disappear, which happens here for $\xi_{2}=0.1$. This is a reflection of the fact that increasing $\xi_{2}$ changes the second eigenvalue $\lambda_{2}^{ \pm}=-\xi_{2} \omega_{2} \pm i \omega_{2} \sqrt{1-\xi_{2}^{2}}$, so that the eigenvalues of the damped system stem apart from the resonance condition so that the discontinuity due to the small denomitar smoothens. 
From this study, it can be concluded that a careful prediction of the type of non-linearity must include the damping in the analysis. Examples on continuous structures (imperfect plates dans spherical shells), are shown in [52], underlining that the results obtained here with the two-dofs system generalize.

\subsection{Closing remarks}

A general strategy has been proposed for deriving the type of nonlinearity of an assembly of nonlinear oscillator equations, based on normal form theory and reduction to a single NNM. With this method, the same complexity is at hand as compared to predictions given by using a single linear mode, as a single oscillator equation is used for the prediction, so that analytical results are derived without resorting to time-consuming numerical simulations including the $N$ oscillator equations. As shown by several authors, see e.g. [33, 31, 32, 55], using a single LNM can lead to erroneous results in the prediction. The reason is the non invariance of the linear eigenspace, as shown in the previous section. The effect of the other modes on the type of nonlinearity, has been underlined, in particular the presence of 2:1 internal resonance.

This section has revealed that using NNMs for reduced-order modeling appears as an appealing method. Indeed, one is at least ascertained to predict the correct type of nonlinearity with the reduced model. For the last section of this lecture, the focus is on the derivation of ROMs for structural systems in forced vibration. The case of harmonic forcing, in the vicinity of one eigenfrequency, is studied. This will allows to demonstrate the ability of ROMs based on NNMs to properly recover a complete bifurcation diagram.

\section{Reduced-order models for resonantly forced response}

The goal of this section is to use the reduced-order modelling strategy based on NNMs and normal form theory, in order to compute the harmonically forced response of thin structures, vibrating at large amplitudes and excited in the vicinity of one of its eigenfrequency. Applications to shells will be specifically shown, and a comparison with the Proper Orthogonal Decomposition (POD) method will highlight the benefit of using NNMs in this case. In the first subsection, the derivation of the ROM is briefly reviewed and the advantage of using the normal form method including the damping is shown on a two-dofs example. The presentation in subsection 4.1 recalls some of the results published in [50]. Subsection 4.2 selects one of the examples shown in [51] for illustration. Subsection 4.3 with application to a closed circular cylindrical shell is taken from [50], while the comparison with the POD method in the last subsection is published in [8].

\subsection{Derivation of the reduced-order model}

The previous developments have clearly highlighted the general method for deriving the ROM, shown schematically in Fig. 8. From a Partial Differential Equation, the first step is a projection onto the basis of the linear eigenmodes. The second step is to use the normal transform in order to express the dynamical equations in an invariant-based span of the phase space. The last step consists in truncating the system by keeping the resonnant NNMs. All the examples used consider the case of a harmonic external forcing, the frequency of which is in the vicinity of an eigenfrequency of the selected structure. In the simplest case, when no internal resonance is present among the eigenfrequencies of the shell, only the NNM whose eigenfrequency is near the excitation, has to be kept in the truncation, so that a very simple ROM, consisting of a single oscillator-equation, is derived. This case will be illustrated in subsection 4.2 with a doubly-curved panel. If internal resonance exists, then all the NNMs, the frequencies of which are contained in the internal resonance, must be kept in the truncation. This case will be illustrated in subsection 4.3, where the ROM of an asymmetric mode of a closed circular cylindrical shell, is considered. Due to the rotational symmetry of the circular shell, asymmetric modes appears by pairs of companion 
modes, in the same manner as what has been observed in circular plates and shells in subsection 3.3. Hence a 1:1 internal resonance is present and two NNMs must be retained in the truncation.

Two main assumptions are thus retained in the derivation of the ROM:

- A third-order asymptotic expansion is used for the normal transform. Hence all the results are a priori accurate up to order three. In particular, the proposed method shows the best results for systems containing quadratic and cubic nonlinearities, as the correction brought by the quadratic term on the cubic nonlinearities is well taken into account. For systems containing only cubic nonlinearity, the improvement as compared to linear mode truncation, is important for the transformation only, but not for the dynamical solutions. When considering large amplitude vibrations, this third-order limitation can become problematic in terms of accuracy of solutions.

- Application of the proposed ROMs to real situations leads to consider external forces applied to the structure. On the mathematical viewpoint, external forces must be taken into account in the normal form computation, as proposed for example in [15]. However, it overshoots the mark of the present study, since the formulation must turn to definitions of time-dependent invariant manifolds. In the mechanical context, time-dependent manifolds have been computed e.g. in [24], requiring a huge computational effort, since the numerical procedure must be repeated for each forcing frequency. Moreover, a consequence of the numerical procedure is that the results are no more expressed under a differential formulation, which renders parametric studies numerically expensive.

In this study, the ROM will be obtained by adding the external force directly to the normal form. The main advantage is that the calculation derived in section 2 is intrinsical to the structure, whereas rigorous computations including the external force must be done for each type of forcing studied. Secondly, the perturbation brought by the external force onto the normal form is at least a second-order effect [21]. Hence, this first approximation will be used to derive simple ROMs, and the results presented in the next sections shows that qualitative and quantitative results are generally obtained.

Before applicating the method to thin shells, a justification for using the normal form with damping is in order. Indeed, the same reasoning made for the external forcing could also have justified a more simple treatment of the damping terms. The ROMS could have been built for the system without damping, using the formulas exposed in subsection 2.4, and then damping and external forces could have been added directly to the normal form. This method will be coined the "conservative NNM" method in the following, and will be compared to the second strategy, termed "damped NNM" formalism, where the damping is included in the normal transform, as shown in subsection 2.8. For that purpose, the two-dofs example consisting of the mass connected to the two nonlinear springs, is used for illustration. The equations of motion, given in Eqs. (42), are completed by adding damping and forcing terms, and reads:

$$
\begin{aligned}
& \ddot{X}_{1}+\omega_{1}^{2} X_{1}+2 \xi_{1} \omega_{1} \dot{X}_{1}+\frac{\omega_{1}^{2}}{2}\left(3 X_{1}^{2}+X_{2}^{2}\right)+\omega_{2}^{2} X_{1} X_{2}+\frac{\omega_{1}^{2}+\omega_{2}^{2}}{2} X_{1}\left(X_{1}^{2}+X_{2}^{2}\right)=F_{1} \cos (\Omega t), \\
& \ddot{X}_{2}+\omega_{2}^{2} X_{2}+2 \xi_{2} \omega_{2} \dot{X}_{2}+\frac{\omega_{2}^{2}}{2}\left(3 X_{2}^{2}+X_{1}^{2}\right)+\omega_{1}^{2} X_{1} X_{2}+\frac{\omega_{1}^{2}+\omega_{2}^{2}}{2} X_{2}\left(X_{1}^{2}+X_{2}^{2}\right)=0 .
\end{aligned}
$$

The forcing is considered on the first mode only, and the excitation frequency is such that $\Omega \approx \omega_{1}$.

Three different truncations, having the same complexity (a single nonlinear oscillator equation), are used as reduced-order models. The first one is the most simple, and consists in keeping only the linear mode by imposing $X_{2}=0$ :

$$
\ddot{X}_{1}+\omega_{1}^{2} X_{1}+2 \xi_{1} \omega_{1} \dot{X}_{1}+\frac{3 \omega_{1}^{2}}{2} X_{1}^{2}+\frac{\omega_{1}^{2}+\omega_{2}^{2}}{2} X_{1}^{3}=F_{1} \cos (\Omega t)
$$


The second ROM, following the "conservative NNM" method, is found by subsituting in Eqs. (45) and (61) the coefficients by their expressions. It reads:

$$
\ddot{R}_{1}+\omega_{1}^{2} R_{1}+2 \xi_{1} \omega_{1} \dot{R}_{1}+\left[\frac{\omega_{2}^{2}-2 \omega_{1}^{2}}{2}+\frac{\left(2 \omega_{1}^{2}-\omega_{2}^{2}\right)}{2\left(\omega_{2}^{2}-4 \omega_{1}^{2}\right)} \omega_{2}^{2}\right] R_{1}^{3}+\left[-3+\frac{\omega_{2}^{2}}{\omega_{2}^{2}-4 \omega_{1}^{2}}\right] \dot{R}_{1}^{2} R_{1}=F_{1} \cos (\Omega t) .
$$

Finally, the third ROM is found by applying the "damped NNM" strategy. It is given by Eq. (81), which is here recalled for $p=1$ :

$$
\ddot{R}_{1}+\omega_{p}^{2} R_{1}+2 \xi_{1} \omega_{1} \dot{R}_{1}+\left(h_{111}^{1}+A_{111}^{1}\right) R_{1}^{3}+B_{111}^{1} R_{1} \dot{R}_{1}^{2}+C_{111}^{1} R_{1}^{2} \dot{R}_{1}=F_{1} \cos (\Omega t),
$$

where $A_{111}^{1}, B_{111}^{1}$ and $C_{111}^{1}$ have not been replaced by their complete expressions for that particular problem as their expressions are now too lengthy. Note however that, as compared to (85), these coefficients now depends explicitely on the damping. In Eq. (85) the only term producing an energy loss is $2 \xi_{1} \omega_{1} \dot{R}_{1}$, whereas in (86), the additional term $C_{111}^{1} R_{1}^{2} \dot{R}_{1}$ is also dissipative, and depends on $\xi_{1}$ and $\xi_{2}$, so that a better approximation of the whole damping in the system is awaited for the second ROM based on "damped NNMs".

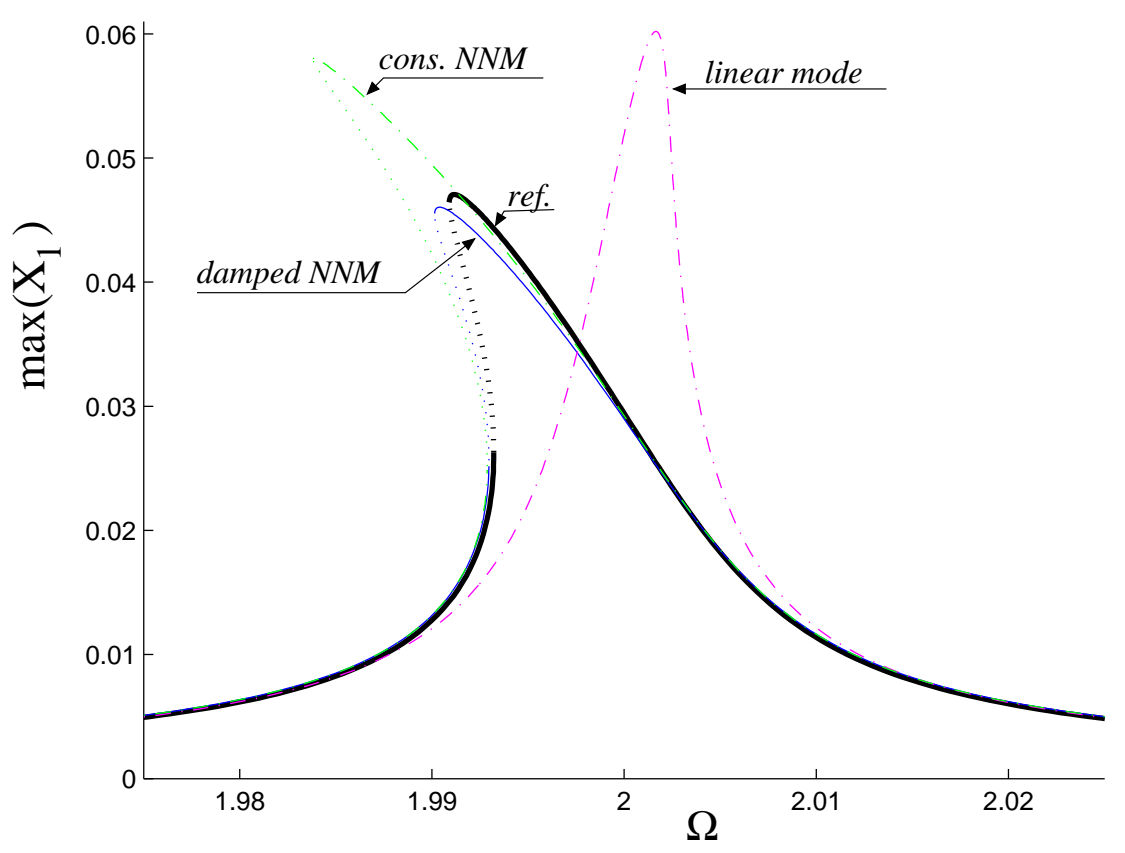

Figure 22: Frequency-response curves (maximum of the first coordinate $X_{1}$ versus excitation frequency $\Omega$ ). Thick solid line : reference solution. Thin blue solid line :"damped NNM", Eq. (86). Dash-dotted green line : "conservative NNM", Eq. (85). Magenta dash-dotted thin line: linear mode truncation, Eq. (84). Selected values: $\omega_{1}=2, \omega_{2}=4.5, \xi_{1}=0.001, \xi_{2}=0.01, F_{1}=5.10^{-4}$.

The performance of the three ROMs are compared on Fig. 22, where the parameter values of the system have been set to $\omega_{1}=2, \omega_{2}=4.5, \xi_{1}=0.001, \xi_{2}=0.01$, so as to simulate the presence of a damped mode and its influence on the frequency-response curve of the first mode. The forcing is such that $\Omega \sim \omega_{1}$, and the amplitude is set as $F_{1}=5 \cdot 10^{-4}$ for that first simulation. The solutions of each model is obtained by continuation of periodic orbits, and the software AUTO is used for that purpose. The reference solution is of course obtained with the complete system, Eqs. (83). One can see that the linear mode truncation predicts an incorrect hardening behaviour, in the line of the results already obtained in the precedent section, and is thus not reliable. On the other hand, the two ROMS based on NMMs 
predict the correct type of nonlinearity. The ROM constructed with the "conservative NNM" method underestimates the damping in the system, and gives a maximum amplitude which is slightly larger that the reference solution. This means that taking into account only $2 \xi_{1} \omega_{1} \dot{R}_{1}$ as dissipative term in the ROM is an incorrect estimation of the whole damping present in the system. The ROM constructed with the "damped NNM" method gives a very satisfactory result. The slight differences observed with the reference solution can be attributed to the two main assumptions made in the construction of the ROM, i.e. the third-order asymptotic development and the time-independent approximation of the manifold.

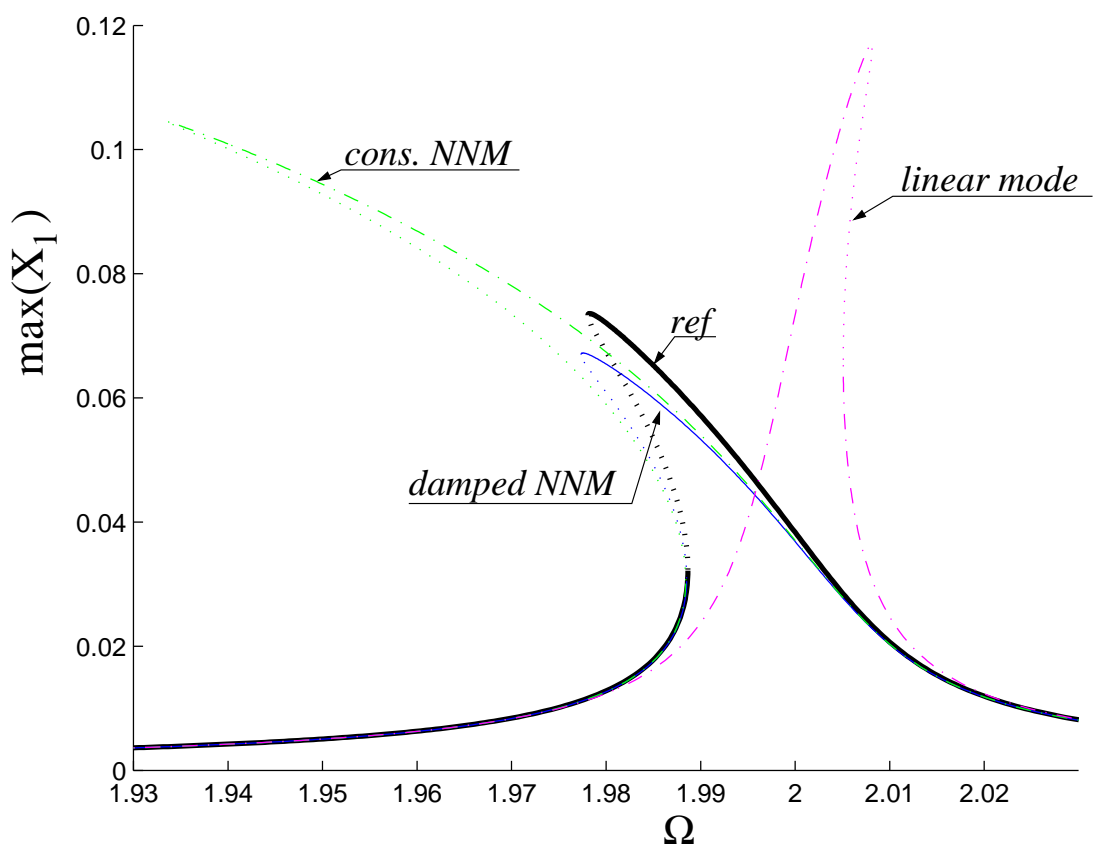

Figure 23: Frequency-response curves (maximum of the first coordinate $X_{1}$ versus excitation frequency $\Omega$ ). Same as Fig. 22, except the amplitude of the forcing which have been increased: $F_{1}=1.10^{-3}$.

These trends are enhanced when the amplitude of the forcing is increased to $F_{1}=1.10^{-3}$, as shown in Fig. 23. In this case, the two ROMs: linear and "conservative NNM" give unaceptable result. The ROM constructed with the "damped NNM" method shows a correct behaviour, even though the slight discrepancies already observed for $F_{1}=5.10^{-4}$ are now clearly observable.

In the remainder of this section, the reduced-order modeling strategy is applied to thin shells including a priori an infinite number of dofs. Truncations to one or two NNMs are realized. As numerous modes, with increasing values of their modal damping factor, will be gathered in a single NNM, the "damped NNM" strategy is systematically used. The examples shown herein underline the importance of having a correct estimation of the damping onto the manifold.

\subsection{Application : the case of a doubly-curved panel with in-plane inertia}

A hyperbolic paraboloid panel with rectangular base, referred to as the HP panel in the following, is first selected. Frequency response curves, in the vicinity of its fundamental mode, will be computed for different ROMs. A sketch of the shell is shown in Fig. 24. The curvilinear coordinate system is denoted by $\left(O, x_{1}, x_{2}, z\right)$, with the origin $O$ at one edge of the panel. $R_{1}$ and $R_{2}$ (assumed to be independent of $x_{1}$ and $x_{2}$ ) are the principal radii of curvature, $a$ and $b$ are curvilinear length, and $h$ is the thickness. The radii of curvature are such that $R_{1}=-R_{2}$. For the numerical results, dimensions of the panel are selected as: $a=b=0.1 \mathrm{~m}, R_{x}=-R_{y}=1 \mathrm{~m}$, and thickness $h=1 \mathrm{~mm}$. The material is linear elastic with Young's modulus $E=206.10^{9} \mathrm{~Pa}$, density $\rho=7800 \mathrm{~kg} . \mathrm{m}^{-3}$ and Poisson's ratio $\nu=0.3$. 


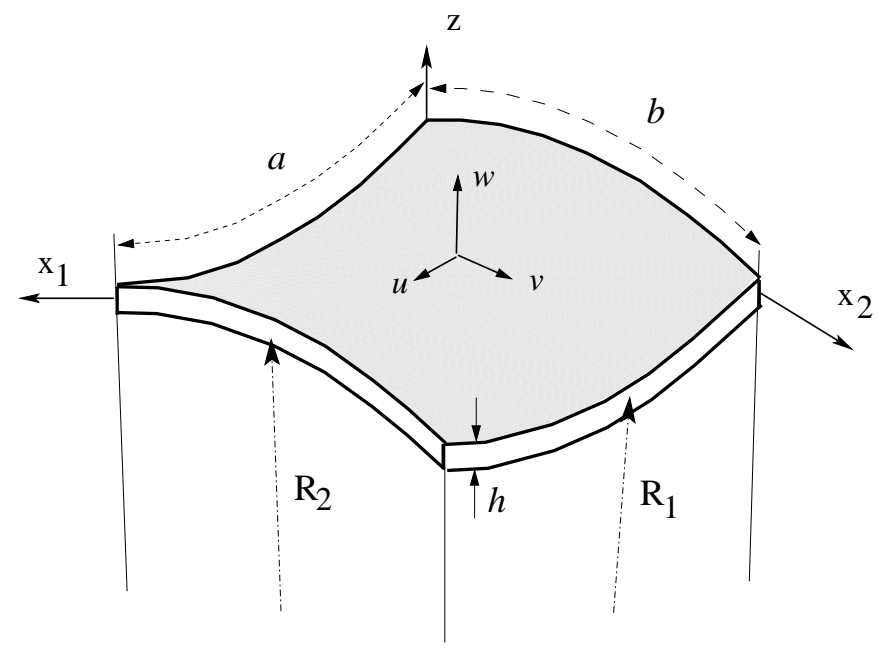

Figure 24: Geometry and coordinate systems for the selected shells.

Donnell's non-linear shell theory is used for expressing the kinematics of the shell. This modelisation is of larger extent than the von Kármán model presented in subsection 3.3. Indeed, Donnell non-linear shell theory do not neglect the in-plane inertia, and can thus be used with confidence for non-shallow shell and on a wider range of displacement amplitudes (and/or a larger range of midfrequencies). The membrane displacements are denoted by $u$ and $v$, and the normal displacement is $w$. Boundary conditions are simply supported.

The drawback of retaining in-plane inertia is that additional degrees of freedom must be taken into the expansion as a consequence that the simplified Donnell's shallow-shell formulation cannot be used. Secondly, the computation of the eigenmodes can become more difficult. For these reasons, ad-hoc expansion functions are here used for discretizing the problem. The basis functions used here are :

$$
\begin{aligned}
& \phi_{m, n}^{(u)}\left(x_{1}, x_{2}\right)=\cos \left(m \pi x_{1} / a\right) \sin \left(n \pi x_{2} / b\right), \\
& \phi_{m, n}^{(v)}\left(x_{1}, x_{2}\right)=\sin \left(m \pi x_{1} / a\right) \cos \left(n \pi x_{2} / b\right), \\
& \phi_{m, n}^{(w)}\left(x_{1}, x_{2}\right)=\sin \left(m \pi x_{1} / a\right) \sin \left(n \pi x_{2} / b\right) .
\end{aligned}
$$

The three displacements are denoted as: $w$ for transverse motions, $u$ and $v$ for in-plane motions. They are expanded as:

$$
\begin{aligned}
u\left(x_{1}, x_{2}, t\right) & =\sum_{m, n=1}^{M_{u}, N_{u}} u_{m n}(t) \phi_{m n}^{(u)}\left(x_{1}, x_{2}\right), \\
v\left(x_{1}, x_{2}, t\right) & =\sum_{m, n=1}^{M_{v}, N_{v}} v_{m n}(t) \phi_{m n}^{(v)}\left(x_{1}, x_{2}\right), \\
w\left(x_{1}, x_{2}, t\right) & =\sum_{m, n=1}^{M_{w}, N_{w}} w_{m n}(t) \phi_{m n}^{(w)}\left(x_{1}, x_{2}\right) .
\end{aligned}
$$

The number of basis functions in each direction is free and governed by the integers $M_{u}, N_{u}, M_{v}, N_{v}, M_{w}$, and $N_{w}$.

Let $\mathbf{q}$ be the vector of generalized coordinates, gathering together all the unknown functions of time introduced by the expansions given in Eqs. (88):

$$
\mathbf{q}=\left[u_{m, n}, v_{m, n}, w_{m, n}\right]^{T}, \quad m=1, \ldots M_{u}, M_{v}, M_{w}, n=1, \ldots N_{u}, N_{v}, N_{w} .
$$


In the remainder, $P$ refers to the dimension of $\mathbf{q}$, i.e. the number of generalized coordinates used for discretizing the shell. The generic element of $\mathbf{q}$ is denoted by $q_{p}$. Finally, in the three considered cases, the result of the discretization gives a set of coupled non-linear oscillator equations to solve. They writes:

$$
\ddot{q}_{p}+2 \zeta_{p} \omega_{p} \dot{q}_{p}+\sum_{i=1}^{P} z_{i}^{p} q_{i}+\sum_{i, j=1}^{P} z_{i, j}^{p} q_{i} q_{j}+\sum_{i, j, k=1}^{P} z_{i, j, k}^{p} q_{i} q_{j} q_{k}=f_{p} \cos (\omega t) .
$$

Modal damping in Eq. (90) is considered in the classical form $2 \zeta_{p} \omega_{p} \dot{q}_{p}$, and $\mathbf{f}=\left[f_{1} \ldots f_{P}\right]^{T}$ is the vector of the projected external forcing considered.

The reference solution is obtained by simulating Eqs. (90). For deriving the bifurcation diagram for the panel (or the frequency response curve in forced vibration), a numerical solution is obtained by using a pseudo-arclength continuation method implemented in the software AUTO [12]. The convergence of the solutions with respect to the number $P$ of generalized coordinates retained has already been done in previous studies. It has been shown that, for the HP panel, $P=22$ basis functions were needed for obtaining convergence [2]. As a consequence of this large value, computation time associated with the numerical simulations with AUTO for obtaining a single frequency-response curve are large.

The first idea for reducing the size of the system is to use the linear normal modes (LNMs). Let $\mathbf{L}=\left[z_{i}^{p}\right]_{p, i}$ be the linear part of Eq. (90), and $\mathbf{P}$ the matrix of eigenvectors (numerically computed) of $\mathbf{L}$ such that: $\mathbf{P}^{-1} \mathbf{L P}=\boldsymbol{\Lambda}$, with $\boldsymbol{\Lambda}=\operatorname{diag}\left[\omega_{p}^{2}\right]$, and $\omega_{p}$ the eigenfrequencies of the structure. A linear change of coordinates is computed, $\mathbf{q}=\mathbf{P X}$, where $\mathbf{X}=\left[X_{1} \ldots X_{P}\right]^{T}$ is, by definition, the vector of modal coordinates. Application of $\mathbf{P}$ makes the linear part diagonal, so that the dynamics can now be expressed in the eigenmodes basis, and reads, $\forall p=1, \ldots, P$ :

$$
\ddot{X}_{p}+2 \zeta_{p} \omega_{p} \dot{X}_{p}+\omega_{p}^{2} X_{p}+\sum_{i, j=1}^{P} g_{i j}^{p} X_{i} X_{j}+\sum_{i, j, k=1}^{P} h_{i j k}^{p} X_{i} X_{j} X_{k}=F_{p} \cos (\omega t) .
$$

The application of $\mathbf{P}$ let the viscous damping unchanged, and $\mathbf{F}=\mathbf{P}^{-1} \mathbf{f}=\left[F_{1} \ldots F_{P}\right]^{T}$ is the new vector of modal forces. The quadratic and cubic non-linear coupling coefficients $\left\{g_{i j}^{p}\right\}$ and $\left\{h_{i j k}^{p}\right\}$ are computed from the $\left\{z_{i, j}^{p}\right\}$ and $\left\{z_{i, j, k}^{p}\right\}$ appearing in Eq (90) with matrix operations involving $\mathbf{P}$. The dimension of $\mathbf{X}$ is $P$, but truncation can now be realized by keeping any number of LNMs. Let $P_{L N M}$ be the dimension of the truncation operated in $\mathbf{X}$. Convergence studies will be realized by increasing $P_{L N M}$ from 1 to $P$. Since the LNMs possesses some interesting properties (in particular orthogonality), it is awaited to obtain convergence for $P_{L N M} \leq P$.

Finally, from Eqs. (91), the NNM method with normal form is applied to obtain a more severely reduced order model. For selecting the number of NNMs for building the ROMs, one needs to know the eigenfrequencies in order to test the presence of internal resonance before proceeding. With dimensions and material properties as chosen, the fundamental frequency is $488.1 \mathrm{~Hz}$. Nondimensionalizing the frequencies by the first one, it results that the list of frequencies are, for the six first: 1, 5.18, 5.18, 9.01, 46.22, 78.05. The fundamental mode (simply supported boundary condition) appears as a "breathing mode" without nodal lines. Then a degenerate eigenvalue with multiplicity two is observed, corresponding to two modes with a nodal line either on the $x_{1}$ or $x_{2}$ direction. In particular no simple internal resonance exists between the first mode and the next. Hence the simplest model, including a single NNM, is selected and should be able to catch the main features of the dynamics of the resonant response.

The response of the HP panel to harmonic excitation in the vicinity of the first eigenfrequency $\omega_{1}$ is numerically computed. The convergence of the solution has been carefully studied in [2] for an excitation amplitude $\tilde{f}$ of $4.37 \mathrm{~N}$ applied at the center of the panel. It has been shown that 22 basis functions were necessary to obtain convergence. More precisely, the generalized coordinates retained for this reference solution are: $w_{1,1}, w_{1,3}, w_{3,1}, w_{3,3}, u_{1,1}, u_{3,1}, u_{1,3}, u_{3,3}, u_{1,5}, u_{5,1}, u_{3,5}, u_{5,3}, u_{5,5}, v_{1,1}, v_{3,1}$, $v_{1,3}, v_{3,3}, v_{1,5}, v_{5,1}, v_{3,5}, v_{5,3}, v_{5,5}$. The damping parameter $\zeta_{p}$ has been set to 0.004 for each mode: $\forall p=1 \ldots 22, \zeta_{p}=0.004$. 


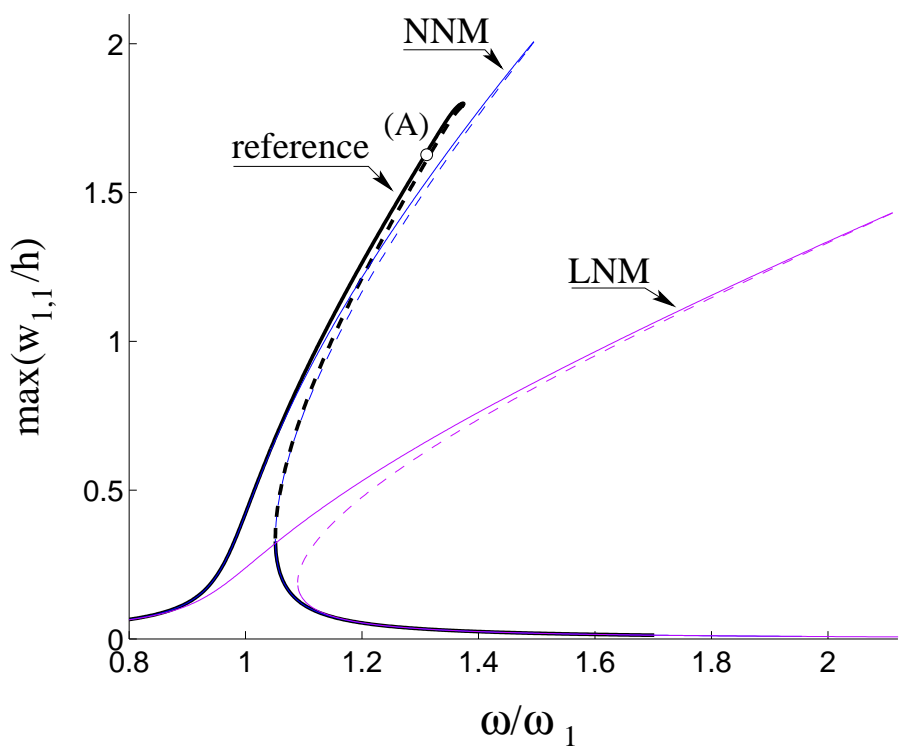

Figure 25: Frequency-response curve for the HP panel, harmonically excited in the vicinity of the first eigenfrequency $\omega_{1}$. The reference solution is compared to the solution given by keeping a single linear mode (LNM) or a single NNM. The excitation amplitude is $\tilde{f}=4.37 \mathrm{~N}$. Point (A), with $\omega=1.3 \omega_{1}$, is used for time integration, see Fig. 27.

Figure 25 shows the frequency-response curve for the reference solution, with 22 basis functions. As a single NNM ROM is awaited to give good results, it is decided to compare two ROMs having the same complexity (a single nonlinear oscillator equation). The first one is obtained by keeping in the truncation only the first LNM $\left(P_{L N M}=1\right)$. Eqs $(91)$ are restricted to the first one :

$$
\ddot{X}_{1}+2 \zeta_{1} \omega_{1} \dot{X}_{1}+\omega_{1}^{2} X_{1}+g_{11}^{1} X_{1}^{2}+h_{111}^{1} X_{1}^{3}=F_{1} \cos (\omega t) .
$$

Branches of solution are numerically obtained by continuation with AUTO, then the original coordinates are recovered via: $\mathbf{q}=\mathbf{P X}$, where, in $\mathbf{X}$, only the first coordinate $X_{1}$ is different from zero.

The second reduced-order model is obtained by keeping the first NNM: Eqs. (52) are truncated by letting $R_{p}=0, \forall p=2 \ldots 22$. The dynamics onto the invariant manifold is then governed by:

$$
\ddot{R}_{1}+2 \zeta_{1} \omega_{1} \dot{R}_{1}+\omega_{1}^{2} R_{1}+\left(h_{111}^{1}+A_{111}^{1}\right) R_{1}^{3}+B_{111}^{1} R_{1} \dot{R}_{1}^{2}+C_{111}^{1} R_{1}^{2} \dot{R}_{1}=F_{1} \cos (\omega t) .
$$

Eq. (93) is solved numerically with AUTO, then one uses Eqs. (39) to come back to the modal coordinates, and finally the matrix of eigenvectors $\mathbf{P}$ allows reconstitution of the amplitudes in the basis of selected projection functions. Thanks to the non-linear nature of the change of variable (39), all the modal amplitudes are non-zero.

Figure 25 shows the main coordinate $w_{1,1}$, having the most significant response. One can observe that the nonlinearity is of the hardening type, and that the amplitude of the response, of the order of two times the thickness, is large. For the ROMs, it is observed that whereas reduction to a single linear mode gives poor result, reduction to a single NNM give a satisfactory result, with a slight overestimation of the maximum vibration amplitude.

Moreover, as shown in Figure 26, the reduced model composed of a single NNM, thanks to the nonlinear change of coordinate, allows recovering all the other coordinates that are not directly excited. Fig. 26 shows the six main coordinates, i.e. the first four coordinates in transverse direction, $w_{1,1}, w_{3,1}, w_{1,3}$ and $w_{3,3}$, as well as the first two longitudinal coordinates $u_{1,1}$ and $v_{1,1}$. It is observed that with the NNM 

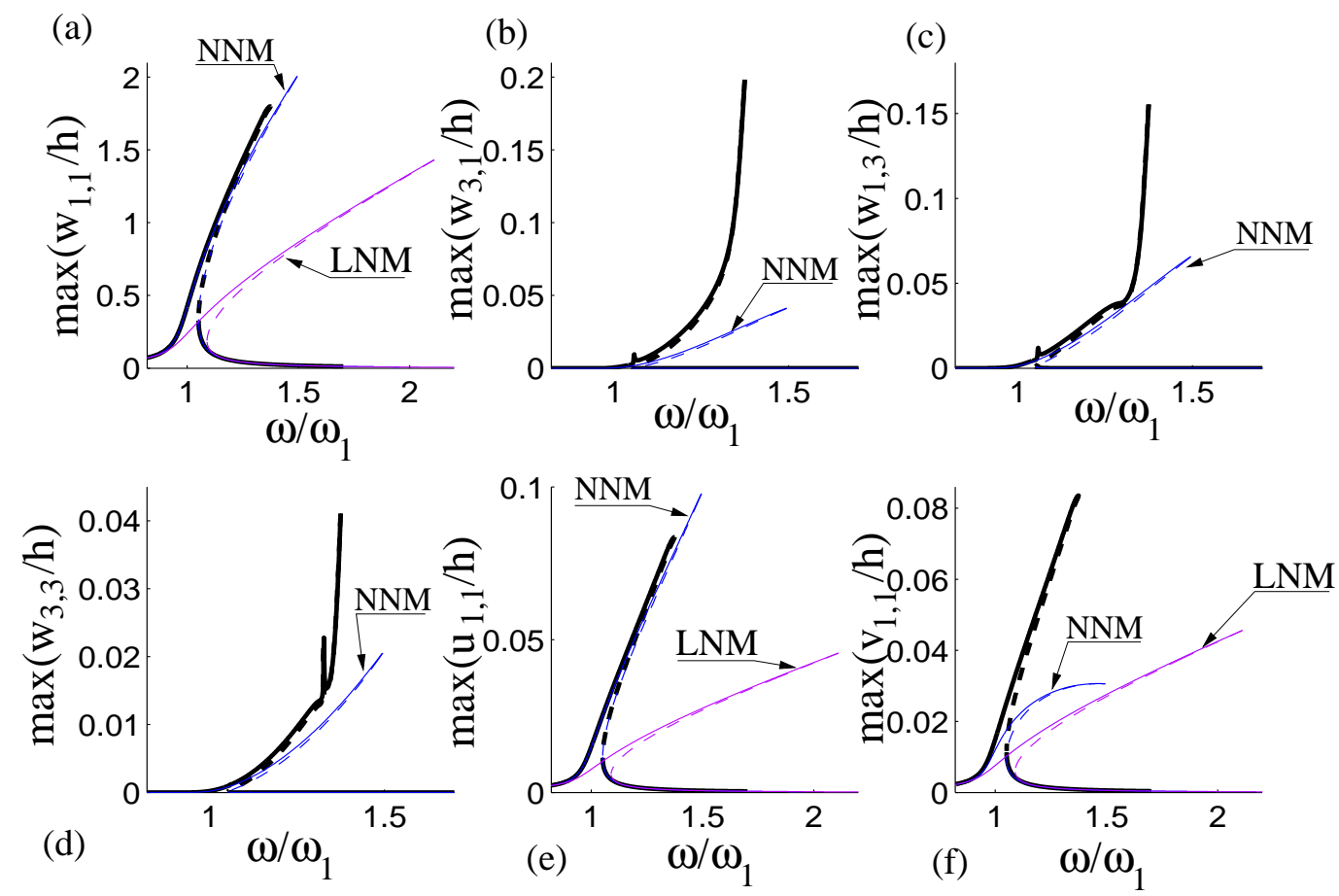

Figure 26: Maximum amplitude of the response of 6 generalized coordinates versus excitation frequency, for an excitation amplitude of $\tilde{f}=4.37 \mathrm{~N}$. Reference solution (thick line) is compared to the reduction to a single linear mode (LNM) and a single non-linear mode (NNM). (a): maximum of $w_{1,1}$. (b): maximum of $w_{3,1}$. (c): maximum of $w_{1,3}$. (d): maximum of $w_{3,3}$. (e): maximum of $u_{1,1}$. (f): maximum of $v_{1,1}$.

ROM, energy is recovered in all the coordinates, with a good approximation of the original amplitudes. On the other hand, for the model composed of a single linear mode, non-zero amplitudes are recovered only on $w_{1,1}, u_{1,1}$ and $v_{1,1}$, as these three coordinates are linearly coupled to create the first eigenmode described by $X_{1}$ which is simulated. But a vanishing response is found with this LNM ROM for $w_{3,1}$, $w_{1,3}$ and $w_{3,3}$.

This first result emphasizes the main characteristic of the NNM ROM : the geometrical complexity due to the curvature of the invariant manifold, is first computed in the non-linear change of coordinates. Once the dynamics reduced to the manifold, a single oscillator equation is sufficient to recover the dynamics. Then, coming back to the original coordinates allows recovering energy onto the slave modes thanks to the non-linear projection.

The time solutions for the four most significant coordinates is shown in Fig. 27. Once again, the reference solution is compared to the two reduced models composed of a single linear and non-linear mode. Time integrations have been performed for $\tilde{f}=4.37 \mathrm{~N}$ and $\omega=1.3 \omega_{1}$ (Point (A) on Fig. 25). Whereas the reduction to a single linear mode is not acceptable, the solutions provided by a single NNM are very good. Despite the fact that only one oscillator-equation is simulated, a variety of complex signals are recovered thanks to the non-linear change of coordinates.

The convergence of the solution with an increasing number of LNMs is shown in Fig. 28 for the excitation amplitude of $4.37 \mathrm{~N}$. It is found that the convergence is very slow : $15 \mathrm{LNMs}$ are necessary to obtain an acceptable solution. The solution with 11 LNMs is qualitatively different from the converged solution with a strange loop appearing in the frequency response, and is thus not acceptable. Hence a very slow convergence with respect to increasing $P_{L N M}$ is found, and using the linear normal modes is not very favourable as compared to the projection functions used. On the other hand, it has been found that increasing the number of NNMs kept in the truncation in Eqs (52) do not change anything in the 

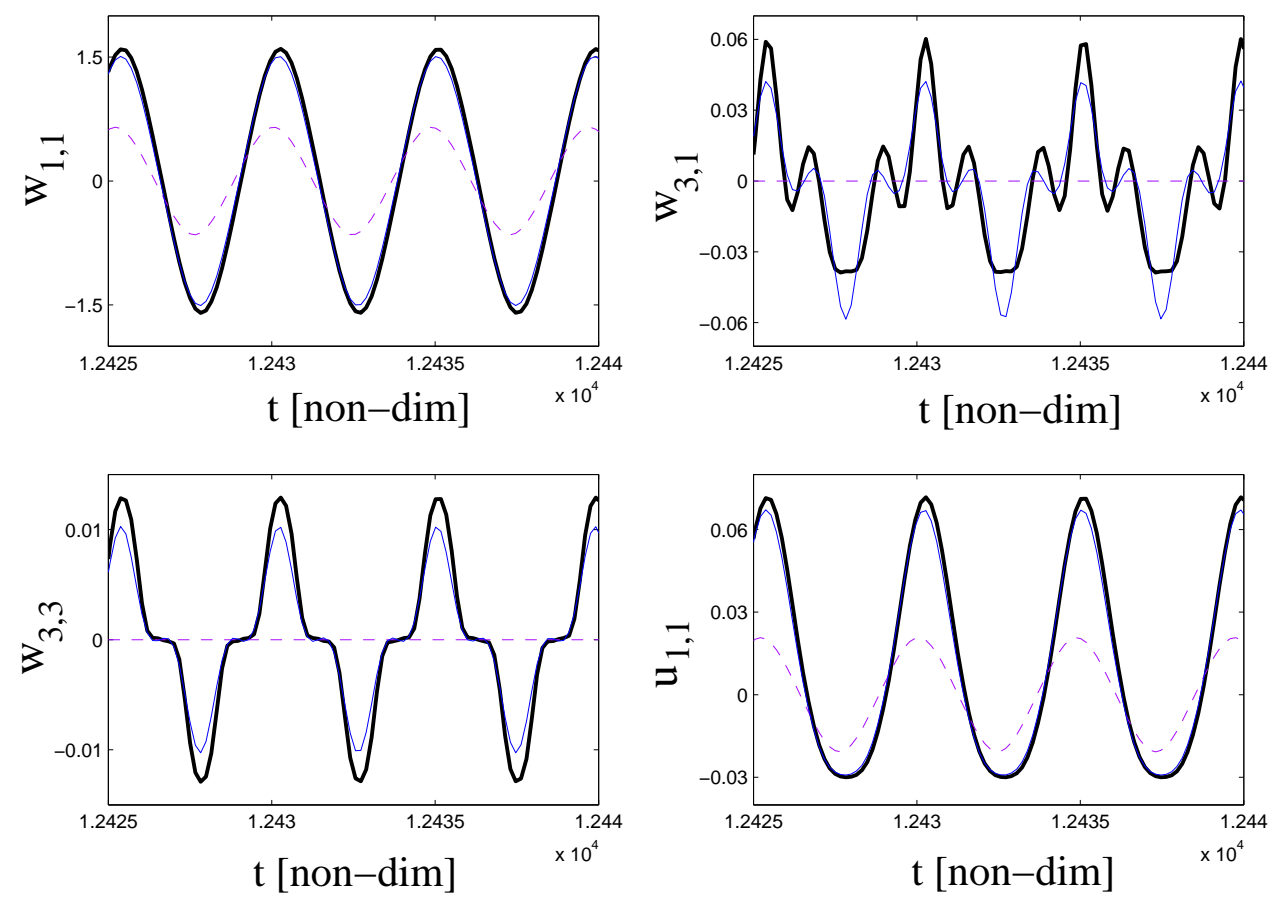

Figure 27: Time-domain response of 4 generalized coordinates of the HP panel, excitation frequency $\omega=1.3 \omega_{1}$, amplitude $\tilde{f}=4.37 \mathrm{~N}$. Reference solution (thick line) is compared to the NNM solution (thin line), and the LNM solution (dashed line).

solution : the added NNMs have been found to stay with constant neglectable amplitude, and the same solution is found as the one obtained with a single NNM. This is a logical consequence of the invariance property of the NNMs. Hence the solution with a single NNM seems to be the best ROM possible. The only way to improve the results found here is not in increasing the number of NNMs, but in overshooting the two limitations of the present approximation used for generating the NNMs.

Finally, the robustness of the ROMs with respect to increasing the amplitude of the forcing, is studied. Fig. 29 shows the results obtained for a lower excitation amplitude: $\tilde{f}=2.84 \mathrm{~N}$, and for a larger one: $\tilde{f}=6.62 \mathrm{~N}$. For $\tilde{f}=2.84 \mathrm{~N}$, the result given by the NNM ROM is almost perfectly coincident with the reference solution obtained with 22 basis function, whereas the model with a single linear mode give unacceptable results. For the larger amplitude, $\tilde{f}=6.62 \mathrm{~N}$, the result deteriorates for the NNM-reduced model, which is not able to catch the saturation loop found by the reference solution at the top of the frequency-response curve. The observation of the other coordinates (not shown for the sake of brevity) shows that this loop reflects the fact that most of the energy is, at this point, absorbed by the higher modes, the amplitude of which significantly and abruptly increase. More precisely, it appears that an internal resonance appears between nonlinear frequencies of the system. Indeed, as the frequencies vary with amplitude, they can fulfill a resonance relationship for a given vibration amplitude even though the linear frequencies are not commensurate. The phenomena encountered here for this level of vibration appears to be of this type with an important increase of energy exchanged from the fundamentally excited modes to higher modes. Hence the reduced model should be changed to catch this new phenomenon, but this appears to be over the scope of the present study.

As a conclusion on the HP panel, the dynamics has been reduced from 22 dofs to a single NNM. Results shows that the reduction, computed with an asymptotic expansion to approach the invariant manifold, gives very good results for vibration amplitudes up to 1.5 times the panel thickness $h$. Beyond 


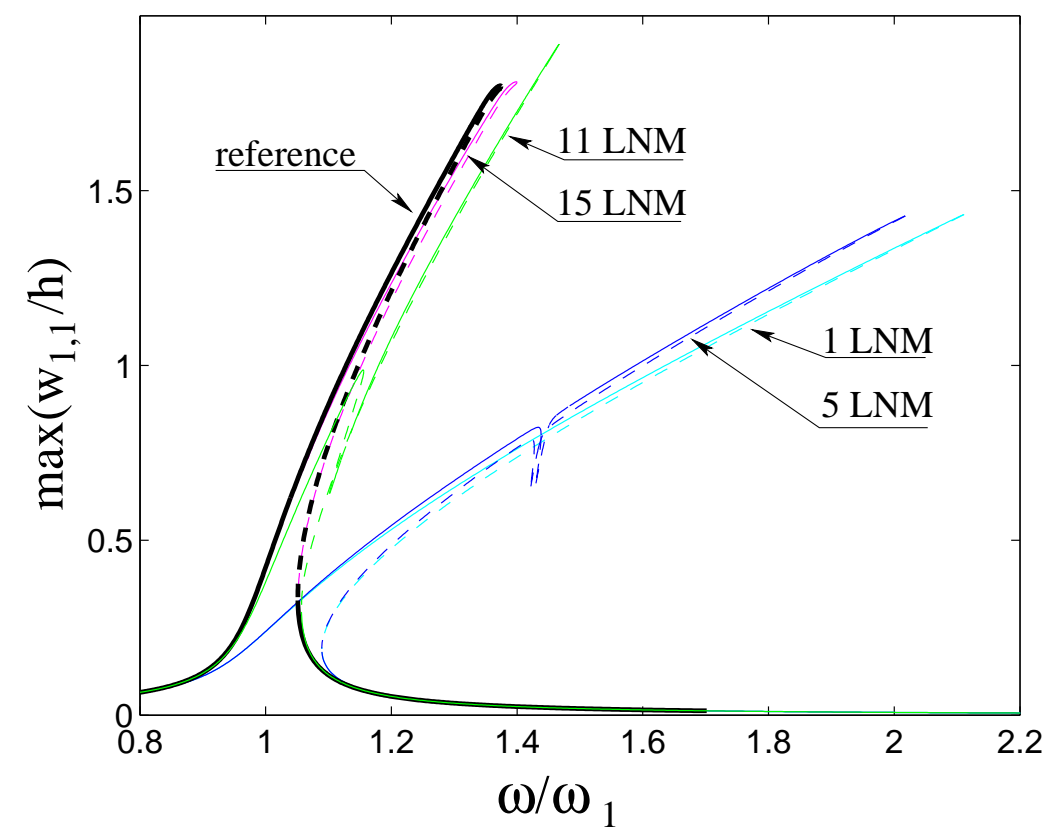

Figure 28: Maximum amplitude response of $w_{1,1}$ versus excitation frequency for $\tilde{f}=4.37 \mathrm{~N}$, showing the convergence of the solution when increasing $P_{L N M}$. Reference solution (22 basis functions) is compared to truncations with 1 linear mode, 5 LNM, 11 LNM and 15 LNM.

(a)

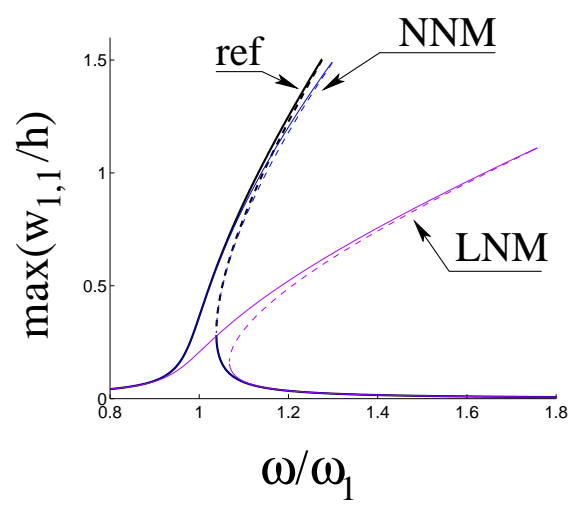

(b)

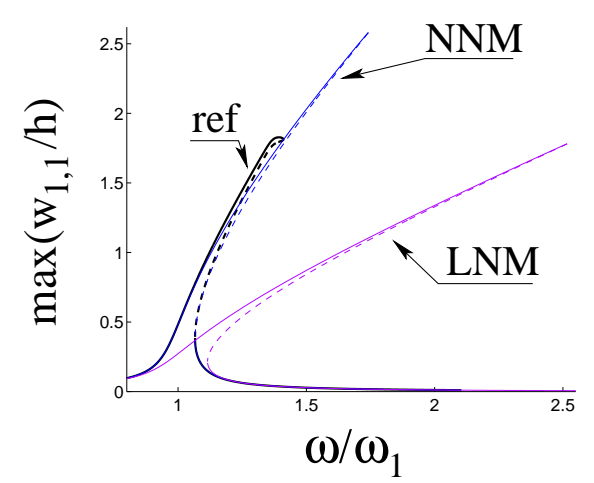

Figure 29: Frequency-response curve for (a): $\tilde{f}=2.84 \mathrm{~N}$, and (b): $\tilde{f}=6.62 \mathrm{~N}$. Reference solution (ref) is compared to truncations with a single linear mode (LNM) and a single non-linear mode (NNM).

this value, the two approximations used for generating the ROM do not hold anymore. On the other hand, using truncations with LNMs did not allow substantial improvement as compared to the selected basis functions used for discretizing the problem.

\subsection{Application : the case of a closed circular cylindrical shell}

A water-filled perfect circular cylindrical shell, simply supported, and harmonically excited in the neighbourhood of the fundamental frequency, is selected in order to derive a NNM-based ROM for a continuous structure. A detailed discussion on the model can be found in [1,6]. Donnell's non-linear 
shallow-shell theory is used to take into account large amplitude motions, so that in-plane inertia, transverse shear deformation and rotary inertia are neglected. Note that Donnell's shallow-shell theory is equivalent to von Kármán model already used in subsection 3.3, so that the equations of motion have the same form. For the transverse deflection $w(x, \theta, t)$, it reads:

$$
D \nabla^{4} w+c h \dot{w}+\rho h \ddot{w}=f-p+\frac{1}{R} \frac{\partial^{2} F}{\partial x^{2}}+\frac{1}{R^{2}}\left[\frac{\partial^{2} F}{\partial \theta^{2}} \frac{\partial^{2} w}{\partial x^{2}}-2 \frac{\partial^{2} F}{\partial x \partial \theta} \frac{\partial^{2} w}{\partial x \partial \theta}+\frac{\partial^{2} F}{\partial x^{2}} \frac{\partial^{2} w}{\partial \theta^{2}}\right],
$$

where $D$ is the flexural rigidity, $E$ Young's modulus, $\nu$ Poisson's ratio, $h$ the shell thickness, $R$ the mean shell radius, $\rho$ the mass density, $c$ the coefficient of viscous damping, $p$ the radial pressure applied to the surface of the shell by the contained fluid, and $f$ is a point excitation, located at $(\tilde{\theta}, \tilde{x})$ :

$$
f=\tilde{f} \delta(R \theta-R \tilde{\theta}) \delta(x-\tilde{x}) \cos (\omega t) .
$$

$F$ is the usual Airy stress function, which satisfies the following compatibility equation:

$$
\frac{1}{E h} \nabla^{4} F=-\frac{1}{R} \frac{\partial^{2} w}{\partial x^{2}}+\left[\left(\frac{\partial^{2} w}{R \partial x \partial \theta}\right)^{2}-\frac{\partial^{2} w}{\partial x^{2}} \frac{\partial^{2} w}{R^{2} \partial \theta^{2}}\right] .
$$

A circumferentially closed circular cylindrical shell of length $L$ is considered. Mathematical expressions of boundary conditions are given in [6,7]. The contained fluid is assumed to be incompressible, inviscid and irrotational. The expression of $p$, which describes the fluid-structure interaction, is given in [1].

The PDE of motion is discretized by projection onto the natural modes basis. The reference solution, whose convergence has been carefully verified in $[1,36]$, is computed by keeping 16 natural modes. The transverse deflection is thus expanded via:

$$
\begin{gathered}
w(x, \theta, t)=\sum_{\substack{m=1 \\
k=1}}^{3}\left[A_{m, k n}(t) \cos (k n \theta)+B_{m, k n}(t) \sin (k n \theta)\right] \sin \left(\lambda_{m} x\right) \\
\quad+\sum_{m=1}^{4} A_{(2 m-1), 0}(t) \sin \left(\lambda_{(2 m-1)} x\right),
\end{gathered}
$$

where $n$ is the number of circumferential waves, $m$ the number of longitudinal half-waves (for symmetry reasons, only odd values are retained), $\lambda_{m}=m \pi / L ; A_{m, n}(t)$ and $B_{m, n}(t)$ are the generalized coordinates. By use of the Galerkin method, 16 second-order differential equations are obtained. They are in the form of the general equations used as the starting point of this study, Eq. (2). The following correspondence between modal coordinates is used: $A_{1, n}$ and $B_{1, n}$ are $X_{1}$ and $X_{2}, A_{3, n}$ and $B_{3, n}$ are $X_{3}$ and $X_{4}, A_{1,2 n}$ and $B_{1,2 n}$ are $X_{5}$ and $X_{6}, A_{3,2 n}$ and $B_{3,2 n}$ are $X_{7}$ and $X_{8}, A_{1,3 n}$ and $B_{1,3 n}$ are $X_{9}$ and $X_{10}, A_{3,3 n}$ and $B_{3,3 n}$ are $X_{11}$ and $X_{12}$, asymmetric modes $A_{1,0}, A_{3,0}, A_{5,0}$ and $A_{7,0}$ are $X_{13}$ to $X_{16}$. Finally, modal damping is postulated.

The reference solution is obtained for the following shell: $L=520 \mathrm{~mm}, R=149.4 \mathrm{~mm}, h=0.519$ $\mathrm{mm}, E=2.06 .10^{11} \mathrm{~Pa}, \rho=7800 \mathrm{~kg} \cdot \mathrm{m}^{-3}, \rho_{F}=1000 \mathrm{~kg} \cdot \mathrm{m}^{-3}$ (water-filled shell), and $\nu=0.3$. The excitation frequency $\omega$ is set in the vicinity of the fundamental mode $(n=5, m=1)$, whose eigenfrequency is $79.21 \mathrm{~Hz}$. Modal damping $\xi_{1, n}=0.0017$ is assumed. The harmonic point excitation has a magnitude of $3 \mathrm{~N}$ and is located at $\tilde{x}=L / 2$ and $\tilde{\theta}=0$. Finally, the displacements are normalized with respect to the thickness $h$, and the time with respect to the period of the first eigenfrequency $\omega_{1, n}$. The frequency-response curves are numerically obtained with the software AUTO.

The response of the shell in the vicinity of an asymmetric mode is investigated. As a consequence of the rotational symmetry displayed by the shell, asymmetric modes appears by pairs, and 1:1 internal resonance exists between each pair of companion modes. Hence, the minimal model which could capture accurately the dynamics is composed of two NNMs. The ROM is build by applying the non-linear 
change of co-ordinates, Eq. (39), to the dynamical systems, so that after this operation, the dynamics is written in terms of the new coordinates $\left(R_{p}, S_{p}\right)$ that are the continuation of the linear ones. Thanks to the invariance property, the truncation can now be done without losing important informations. Two couples of master coordinates, $\left(R_{1}, S_{1}\right)$ and $\left(R_{2}, S_{2}\right)$ are selected, which are related to $\left(A_{1, n}, \dot{A}_{1, n}\right)$ and $\left(B_{1, n}, \dot{B}_{1, n}\right)$. All other normal coordinates $\left(R_{p}, S_{p}\right)$, for $p \geq 3$, are set to zero. The normal dynamics with these two master coordinates derives from Eq. (52): one has just to write this system for $p=1,2$. The dynamics onto this four-dimensional invariant manifold is thus governed by:

$$
\begin{aligned}
\ddot{R}_{1}+\omega_{1}^{2} R_{1}+ & \xi_{1} \omega_{1} \dot{R}_{1}+\left(A_{111}^{1}+h_{111}^{1}\right) R_{1}^{3}+B_{111}^{1} R_{1} \dot{R}_{1}{ }^{2} \\
& +\left(A_{212}^{1}+A_{122}^{1}+h_{122}^{1}\right) R_{1} R_{2}^{2}+B_{122}^{1} R_{1} \dot{R}_{2}{ }^{2}+B_{212}^{1} R_{2} \dot{R}_{1} \dot{R}_{2} \\
& +C_{111}^{1} R_{1}^{2} \dot{R}_{1}+\left(C_{122}^{1}+C_{212}^{1}\right) R_{1} R_{2} \dot{R}_{2}+C_{221}^{1} R_{2}^{2} \dot{R}_{1}=f \cos (\omega t) \\
\ddot{R}_{2}+\omega_{2}^{2} R_{2}+ & \xi_{2} \omega_{2} \dot{R}_{2}+\left(A_{222}^{2}+h_{222}^{2}\right) R_{2}^{3}+B_{222}^{2} R_{2} \dot{R}_{2}{ }^{2} \\
& +\left(A_{112}^{2}+A_{211}^{2}+h_{112}^{2}\right) R_{2} R_{1}^{2}+B_{211}^{2} R_{2} \dot{R}_{1}{ }^{2}+B_{112}^{2} R_{1} \dot{R}_{1} \dot{R}_{2} \\
& +C_{222}^{2} R_{2}^{2} \dot{R}_{2}+\left(C_{121}^{2}+C_{211}^{2}\right) R_{1} R_{2} \dot{R}_{1}+C_{112}^{2} R_{1}^{2} \dot{R}_{2}=0
\end{aligned}
$$

where the coefficients $A_{i j k}^{p}, B_{i j k}^{p}$ and $C_{i j k}^{p}$ are given by Eq. (53). In case of low-order internal resonance, the dynamical monoms corresponding to resonant terms should normally be added into the normal form, Eq. (52), which were derived for the case of no internal resonance. However, the case considered here (a perfect shell) does not produce new terms because of the perfect symmetry of the initial problem. For example, one could have find a monom like $R_{1}^{2} R_{2}$ in the first equation as it is a resonant term. However, this dynamical term is not present in the original equation because $h_{112}^{1}=0$, so it is not present in the normal form.

For comparison, the ROM obtained with the "conservative $N N M$ " formulation is also computed. It can be obtained from Eq. (98) by setting $C_{i j k}^{p}=0$, and $A_{i j k}^{p}, B_{i j k}^{p}$ to their values obtained for $\xi_{i}=0, \forall i$. Frequency-response curves are numerically obtained with AUTO for the three following models: reference solution with 16 degrees-of-freedom, and the two ROMs corresponding to "damped" and "conservative" NNMs. For these simulation, the original modal coordinates are simply recovered by using Eq. (39).

Figure 30 shows the frequency-response curves for the driven mode $A_{1, n}$ and its companion mode $B_{1, n}$. The full system simulation with 16 dofs is presented with a thick line. The dynamical response is composed of two main branches of solution. The first branch correspond to single-mode motion where only the driven mode, $A_{1, n}$, is present in the response $\left(B_{1, n}=0\right)$. This branch present a softening-type nonlinearity. Due to the presence of the companion mode in 1:1 internal resonance with the driven mode, the main branch loses stability at the Bifurcation point (BP), where a secondary branch of coupled solutions emerge. Along this secondary branch, the coupling between the two resonant modes is strong and energy is exchanged inbetwwen them, resulting in important values for $B_{1, n}$. Moreover, this secondary branch encounters an instability with the presence of two Neimarck-Sacker bifurcation points, denoted as TR (like "torus" bifurcation) on the figure. Inbetween these two Neimarck-Sacker bifurcations, the harmonic solutions are not stable anymore, and a quasiperiodic response is observed.

Let us now observe how the ROMs build with NNMs are able to recover all the fine details of this bifurcation diagram. The "conservative NNM" case is plotted with a dash-dotted line. One can see that all the dynamical features of the original system are recovered: the two branches are found as well as the nature of the bifurcations and the stability. This result was awaited since it is a fundamental property of the normal form to recover the essential dynamical properties, thus the qualitative behaviour (number and nature of bifurcations) will always be predicted by the ROM. As already mentioned in the 2-dof example, a higher value of the maximum amplitude is found, showing that the damping has been underestimated. This is corrected with the "damped NNM" ROM, which gives a very good result, although one may argue that the softening effect is a little bit overestimated. 

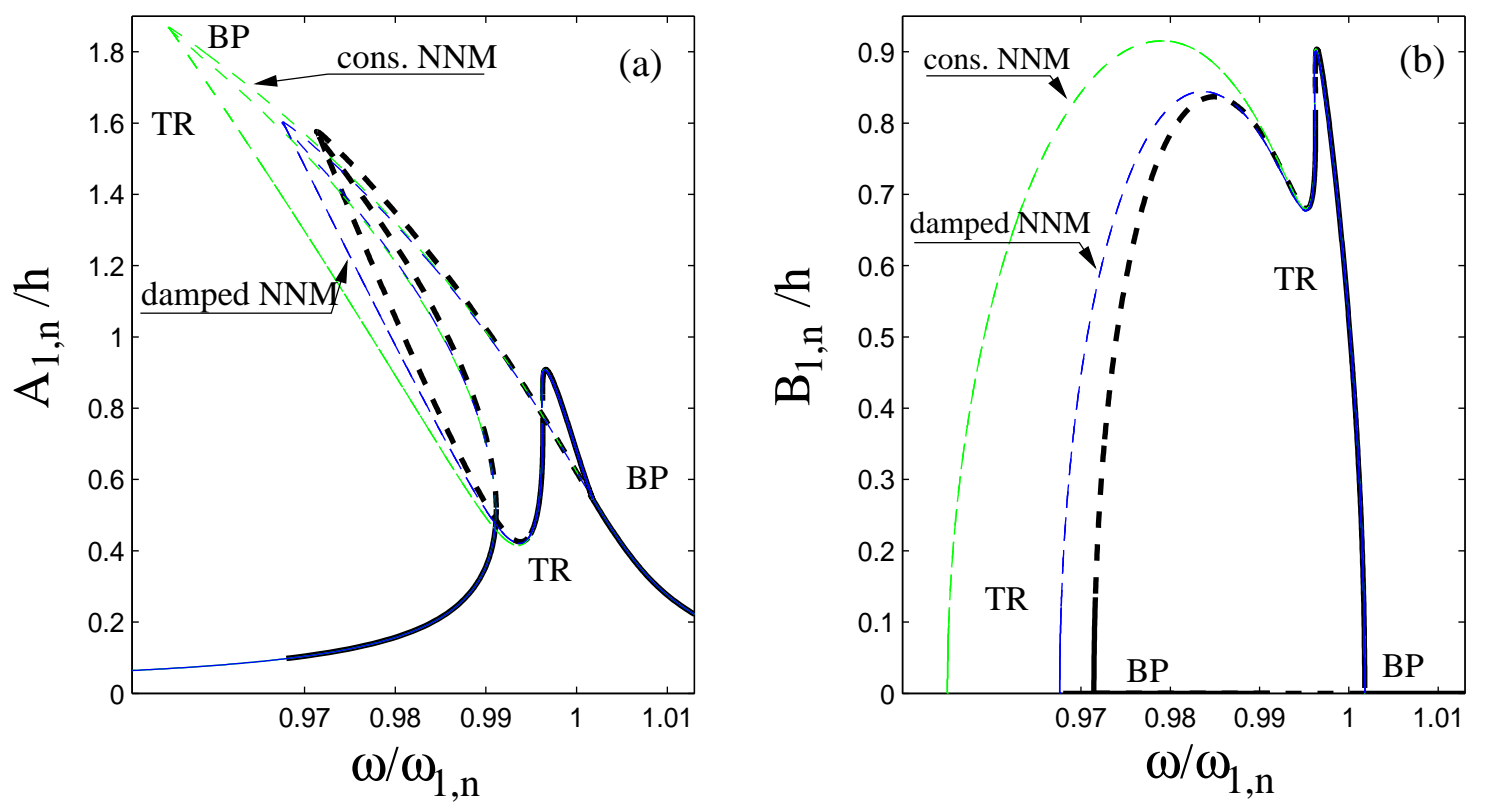

Figure 30: Maximum amplitude of transverse shell vibration versus excitation frequency. (a): driven mode $A_{1, n}$. (b): companion mode $B_{1, n}$. Thick line: reference solution (unstable states (dashed thick line) and bifurcation points are indicated: BP : pitchfork, TR: Neimarck-Sacker (torus) bifuration). Conservative and damped NNM ROMs are represented with thin line.

Recovering the original modal coordinates with Eq. (39) shows that, thanks to the curvature of the invariant manifold in phase space, slight contributions are present onto all others linear modal coordinates. For comparison, the sixth most important modal amplitudes are represented in Fig. 31, for the full-order model, and the "damped NNM" ROM. This figure shows that the reduced model with two equations allows to recover all the modal amplitudes with good accuracy.

This example shows that the method can be easily used for reducing the non-linear dynamics of geometrically non-linear structures. The main advantage relies in the quickness of the method: computing all the coefficients appearing in Eqs. $(39,53,52)$ is immediate on a standard PC for this 16-dof full-order system. As two approximations are used to produce the ROM (namely a time-invariant manifold is used, and it is computed by a third-order asymptotic development), it is awaited that the results can deteriorate for very large amplitude motions. However, this example shows that up to two times the thickness of the shell, the ROM is robust.

\subsection{Comparison with the Proper Orthogonal Decomposition method}

This last section is devoted to a comparison between reduced-order modeling as proposed with NNM via normal form theory, and the more popular Proper Orthogonal Decomposition (POD) method. For that purpose, the case of the circular cylindrical shell, filled with water and harmonically excited in the vicinity of one of its fundamental frequency (as in the previous subsection), is selected. A brief presentation of the POD method is first carried out, then some results are presented in order to illustrate the advantages and drawbacks of each method.

The POD method optimally extracts the spatial information necessary to characterize the spatiotemporal complexity and inherent dimension of a system, from a set of temporal snapshots of the response, gathered from either numerical simulations or experimental data. This point is important in the comparison since one needs to have at hand a set of solutions of the system, in order to build the POD ROM from that set. This can be seen as an advantage in an experimental context, where the POD method 

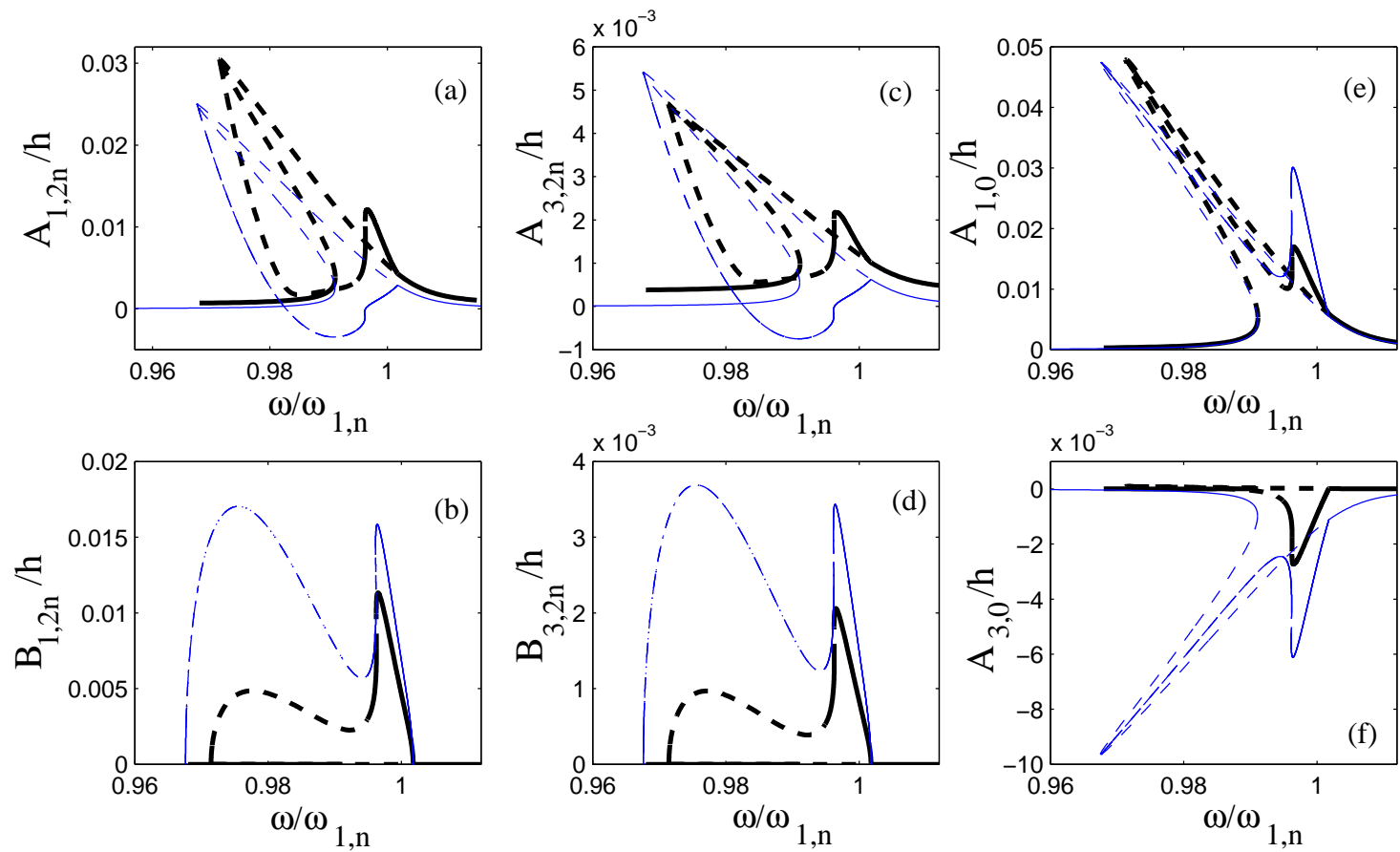

Figure 31: Maximum amplitude of transverse shell vibration versus excitation frequency for six slave modal coordinates: (a) and (b): driven and companion of asymmetric $(1,2 \mathrm{n})$ mode; (c) and (d): driven and companion of asymmetric $(3,2 \mathrm{n})$ mode; (e) and (f): axisymmetric $(1,0)$ and $(3,0)$ modes. Thick line: reference solution. Thin line : ROM computed from the two-dof "damped NNM" master coordinates.

is routinely used to extract e.g. coherent structures from measurements. However in a theoretical context, this appears as a drawback, since one is obliged to run some simulation to have a set of results in order to build the POD ROM. Moreover, the question of the choice of the dynamical solutions to compute, from which the ROM is built, is very important, and a great care must be taken in this choice.

In the present context, the temporal responses are obtained via direct simulation with the conventional Galerkin solution. The question of the selected dynamical solutions for these direct solutions is postponed to the results to better explain the differences one can obtain in choosing e.g. either a regular or a chaotic solution. The Proper Orthogonal Modes (POMs) obtained by the POD method are denoted $\Psi_{i}(r, \theta)$. Once they are obtained, a Galerkin approach will be used in order to derive oscillator equations for the ROM, via:

$$
w(r, \theta, t)=\sum_{i=1}^{P_{P O D}} a_{i}(t) \Psi_{i}(r, \theta) .
$$

In this expression, $a_{i}(t)$ are the proper orthogonal coordinates and $P_{P O D}$ is the number of POMs (dof) used to build the POD model. It is awaited to obtain $P_{P O D}$ significantly smaller than $P_{L N M}$, the number of linear modes necessary for the conventional Galerkin method to converge. The question is here to compare $P_{P O D}$ with $P_{N N M}$.

Let us now explain briefly the method used to find out the POD modes $\Psi_{i}(r, \theta)$. The interested reader is referred to general introduction on the POD method to have a complete description, see e.g. $[9,17$, $26,27]$, here only the main steps are recalled. The displacement field $w(r, \theta, t)$ is first divided into its time-mean value $\bar{w}(r, \theta)$ and the zero-mean response $\tilde{w}(r, \theta, t)=w(r, \theta, t)-\bar{w}$. In the POD method, the POMs are obtained by minimizing the objective function defined by:

$$
\tilde{\lambda}=<\left(\Psi_{i}(r, \theta)-\tilde{w}(r, \theta, t)\right)^{2}>, \quad \forall(r, \theta) \in \Omega
$$


where $\Omega$ denotes the space domain, $<>$ the time-averaging operation and $\Psi_{i}(r, \theta)$ the generic POD mode. For minimizing the objective function, a collection of dynamical states are needed. They are collected from a direct simulation. For example, having realized a temporal solution of the complete problem for a given external force, resulting in a given dynamical behaviour, one is led with a discrete collection of events $\tilde{w}\left(r, \theta, t_{n}\right)$, for $n=0$ to $N_{t}$, the number of time steps of the dynamical simulation. Let us denote by $\tilde{w}_{n}$ the so-called temporal snapshots of the computed solution $\tilde{w}\left(r, \theta, t_{n}\right)$. Then, the time-averaging operation of a series of $N_{t}$ snapshots writes:

$$
<\tilde{w}(r, \theta, t)>=\frac{1}{N_{t}} \sum_{n=1}^{N_{t}} \tilde{w}_{n}
$$

Minimizing of the objective function (100) is obtained, after some mathematics, by solving the following eigenvalue problem:

$$
\int_{\Omega}<\tilde{w}(r, \theta, t) \tilde{w}\left(r^{\prime}, \theta^{\prime}, t\right)>\Psi\left(r^{\prime}, \theta^{\prime}\right) r^{\prime} \mathrm{d} r^{\prime} \mathrm{d} \theta^{\prime}=\lambda \Psi(r, \theta),
$$

where $<\tilde{w}(r, \theta, t) \tilde{w}\left(r^{\prime}, \theta^{\prime}, t\right)>$ is the time-averaged spatial autocorrelation function.

A Galerkin projection scheme for determining POMs semi-analytically, and in parallel to approximate the solution of the PDE, is now presented. This technique has been developped initially in [40] for beams conveying fluid and extended to circular cylindrical shells in $[6,7,8]$. The main idea is to express the POD modes as linear combination of the eigenmodes and the structures, hence allowing for:

- A physical interpretation of the POMs

- An analytical expression of the dynamical system governing the dynamics of the problem in the POD basis.

The generic POM is projected on the eigenmodes $\Phi_{i}(r, \theta)$ of the shell as:

$$
\Psi(r, \theta)=\sum_{i=1}^{P_{L N M}} \alpha_{i} \Phi_{i}(r, \theta)
$$

where $\alpha_{i}$ are unknown coefficients. Then, the following eigenvalue problem is finally obtained:

$$
\underline{\underline{A}} \underline{\alpha}=\lambda \underline{\underline{B}} \underline{\alpha},
$$

where

$$
A_{i j}=\tau_{i} \tau_{j}<\tilde{q}_{i}(t) \tilde{q}_{j}(t)>, \quad B_{i j}=\tau_{i} \delta_{i j}, \quad \tau_{i}=\int_{\Omega} \Phi_{i}^{2}(r, \theta) r \mathrm{~d} r \mathrm{~d} \theta,
$$

$\delta_{i j}$ is the Kronecker symbol, $\tilde{q}_{i}(t)=q_{i}(t)-\bar{q}_{i}$ is the zero-mean response of the ith generalized coordinate, with $\bar{q}_{i}$ being its mean. The norm of the basis functions $\tau_{i}$ in the present case is $\pi R L / 2$ for asymmetric modes and $\pi R L$ for axisymmetric modes. In Eq. (104), $\underline{\underline{A}}$ and $\underline{\underline{B}}$ are symmetric and positive definite matrices of dimension $P_{L N M} \times P_{L N M}$, and $\underline{\alpha}$ is a vector containing the $P_{L N M}$ unknown coefficients of the POMs. The eigenvectors $\underline{\alpha}$ corresponding to the largest eigenvalues (known as dominant POMs) in Eq. (104) can now be inserted in Eq. (103) that gives a basis for the approximate solution of the PDE using the Galerkin approach; this will be referred to as the POD-Galerkin scheme hereafter.

Recombination of modal expressions given in Eqs (103), (99) and (97) allows deriving the accurate expansion of the POD mode onto the basis of the linear eigenmodes of the shell as:

$w(r, \theta, t)=\sum_{i=1}^{P_{P O D}} a_{i}(t) \sum_{j=1}^{P_{L N M}} \alpha_{j i} \Phi_{j}(r, \theta)=\sum_{i=1}^{P_{P O D}} \sum_{m=1}^{M} \sum_{n=0}^{N}\left[\alpha_{m, n, i} \cos (n \theta)+\beta_{m, n, i} \sin (n \theta)\right] \sin \left(\lambda_{m} r\right)$, 
where on the right-hand side two different symbols, $\alpha_{m, n, i}$ and $\beta_{m, n, i}$, have been introduced to differentiate the coefficients of the POMs for cosine and sine terms in $\theta$ and are given by the corresponding $\alpha_{j i}$. Eq. (106) is used to solve the equations of motion of the shell, given in the beginning of the previous subsection, Eqs. (94) and (96), with the Galerkin method to find the equations of motion of the ROM. Moreover, Eq. (106) has still the same shape over the shell surface as Eq. (97); therefore, the fluid-structure interaction can be treated with the same approach used for the Galerkin method. This is not surprising, because the POD modes have been projected on the eigenmodes.

The coefficients $\alpha_{m, n, i}$ and $\beta_{m, n, i}$ are also meaningful in order to get a physical interpretation of the POMs in phase space. Indeed, the POD method can be seen as a geometrical method that span the phase space with orthogonal modes, as enforced by the linear eigenvalue problem that defines the POMs, Eq. (104). These orthogonal modes are defined by the direction of the phase space where most of the information is present, which also explains why the choice of the data used for computing the POMs is crucial. In the POD-Galerkin scheme developed here, the POMs are expressed in the basis of the linear eigenmodes of the selected structure, so that the coefficients $\alpha_{m, n, i}$ and $\beta_{m, n, i}$ can be directly read as the deviation between the POMs and the linear eigenspaces.
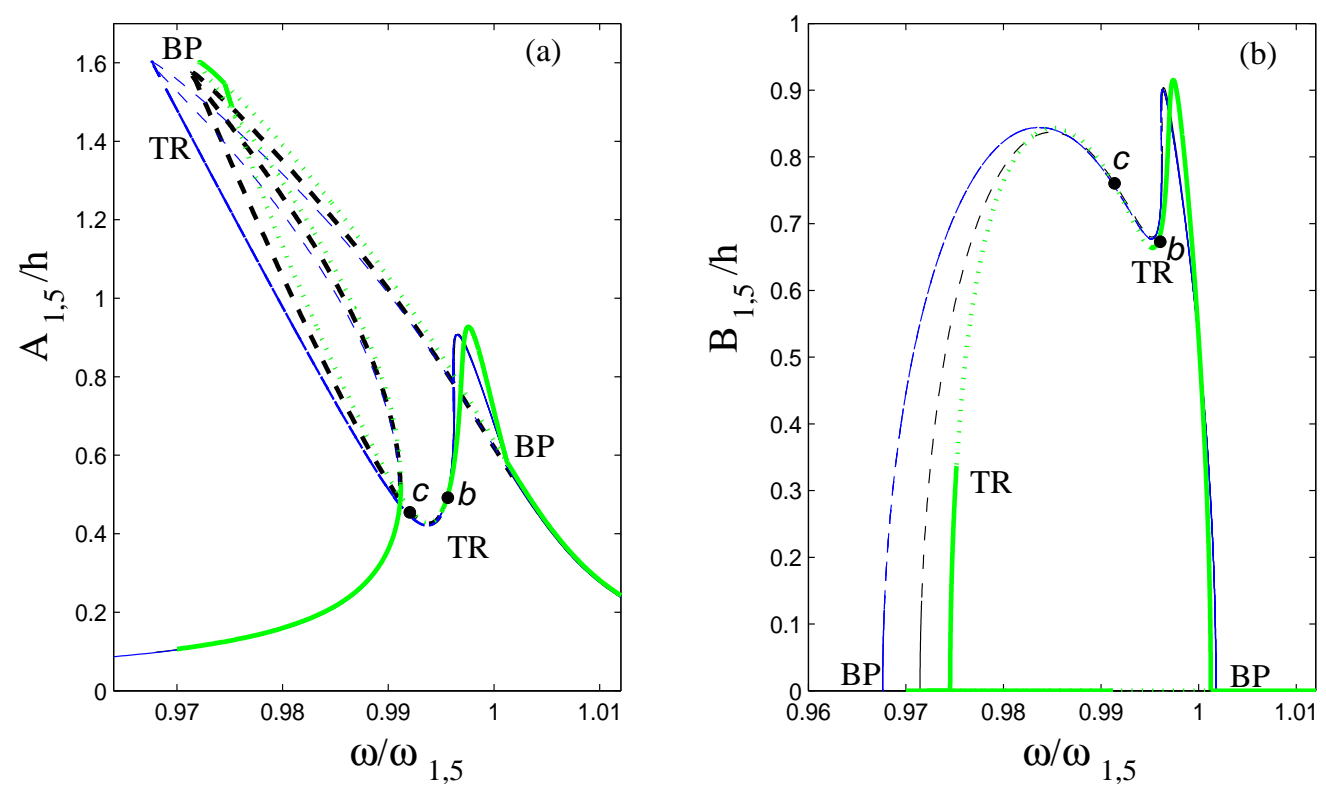

Figure 32: Maximum amplitude of transverse shell vibration versus excitation frequency. (a): driven mode $A_{1, n}$. (b): companion mode $B_{1, n}$. Thick line: reference solution (unstable states (dashed thick line) and bifurcation points are indicated: BP : pitchfork, TR: Neimarck-Sacker (torus) bifuration). Blue thin line: ROM obtained by considering two NNMs. Green thick line: ROM obtained by considering three POMs. Points $b$ and $c$ denotes the dynamical responses used for building the POD ROM.

The comparison between the ROMs either based on NNMs, or on POD, is now addressed. For that purpose, the case of the circular cylindrical shell filled with water, excited harmonically in the vicinity of its fundamental mode, is considered. The performances of the ROMs are assessed by comparing their ability to recover the full bifurcation diagram found in the previous subsection, Fig. 30. Fig. 32 shows the comparison between the reference model with 16 linear modes, as already shown, together with the ROM composed of two NNMs (also shown in the previous section), and finally the result given by considering three POD modes. 


\begin{tabular}{c|cc|cc|c|c}
$i^{\text {th }}$ POM & $\alpha_{1,5, i}$ & $\beta_{1,5, i}$ & $\alpha_{1,10, i}$ & $\beta_{1,10, i}$ & $\alpha_{1,0, i}$ & $\alpha_{3,0, i}$ \\
\hline 1 & 1 & 0 & 0.000213 & 0 & 0.000043 & $8.8 \mathrm{e}-6$ \\
2 & 0 & 1 & 0 & -0.00029 & 0 & 0 \\
3 & 0.000123 & 0 & -0.1847 & 0 & -0.9641 & 0.1855
\end{tabular}

Table 1: Coefficients $\alpha_{m, n, i}$ and $\beta_{m, n, i}$ defined in Eq. (106), for the first three POD modes obtained with point $c$.

The selection of the temporal response used for building the POD-based ROM is fully documented in [6], illustrating the difficulties encountered for choosing a proper case allowing to recover the response shown in Fig. 32. In particular, Fig. 32 shows two points, $b$ and $c$, that have been used in the process. For point $b$, the frequency is $\omega=0.995 \omega_{1}$, the response is on the second branch, which means that the coupling between the two configurations is activated. However, the point $b$ is selected just before the Neimarck-Sacker bifurcation, so that the response is harmonic. On the other hand, point $c$, for $\omega=0.991 \omega_{1}$, is also on the coupled solutions branch, but in the quasiperiodic regime. If one uses point $b$ for building the POD model, results are not satisfactory at all: the solution branches moves off the reference solution [6]. Secondly, it has then been found impossible to recover the Neimarck-Sacker bifurcation point in that case, so that the quasiperiodic regime is not predicted by the POD based ROM.

For the POD ROM, the best result has been obtained by using point $c$, in the quasiperiodic regime. The reason is that the quasiperiodic orbits shows larger variations in phase space. Hence the available information for building the ROM is more important. For reproducing the complete bifurcation diagram, the best solution has been obtained by retaining three POMs, it is shown as a green line in Fig. 32. One can observe that the complete bifurcation diagram has been fairly recovered, with all bifurcation points and special regimes found.

Comparing now the NNM and the POD-based ROMS, one can observe that both are able to recover the bifurcation diagram. However, three POMs are necessary, versus only two NNMs. Retaining only the first two POMs gives incorrect results: in that case, the first bifurcation point allowing to go from the uncoupled, first branch of solution; to the coupled, secondary branch, is not detected, so that 1:1 resonant motions are not predicted! On the other hand, the NNM-based ROM gives very satisfactorily results with only two modes, and with direct application from the PDE of motions, without having to work out with a difficult selection of a correct database to construct the ROM.

A geometrical interpretation, in the phase space, allows to understand why three POMs are necessary to reconstruct the correct solution, whereas only two NNMs are enough. The coefficients $\alpha_{m, n, i}$ and $\beta_{m, n, i}$ for the first three POMs, obtained by using point $c$ (quasiperiodic state) for creating the snapshots database, are given in Table 1 . One can see that $\alpha_{1,5,1}=1$, which means that the first POD mode is very near the first linear eigenspace, with a very slight deviation given by the small, but non-zero, values of $\alpha_{1,10,1}$ and $\alpha_{1,0,1}$. The second line shows that the second POD mode is very near the second linear eigenspace, with a very slight correction along $B_{1,10}$. Finally the third line shows that the third POM is essentially colinear to the first axisymmetric mode $(1,0)$, with some slight corrections.

Figure 33 shows a cut-off (Poincaré section) in the phase space of the complete problem, which is of dimension 33 (16 linear modes with displacement and velocity, plus the forcing term). The plane selected for the section is $A_{(1,5)}, A_{(1,0)}$ : driven mode and first axisymmetric mode. The clouds of points are obtained from the direct simulation obtained with the full model, at points $b$ (harmonic coupled solution) and $c$ (coupled quasiperiodic motion). As awaited, the quasiperiodic motion occupies a larger volume in phase space. The most important appears in the non-negligible motions along the axisymmetric mode. The fundamental importance of retaining axisymmetric modes for analyzing nonlinear vibrations of asymmetric modes of shells has already been underlined in many studies $[4,5,3]$. It is the result of a non-resonant, invariant-breaking term between these two modes. Consequently, the response 


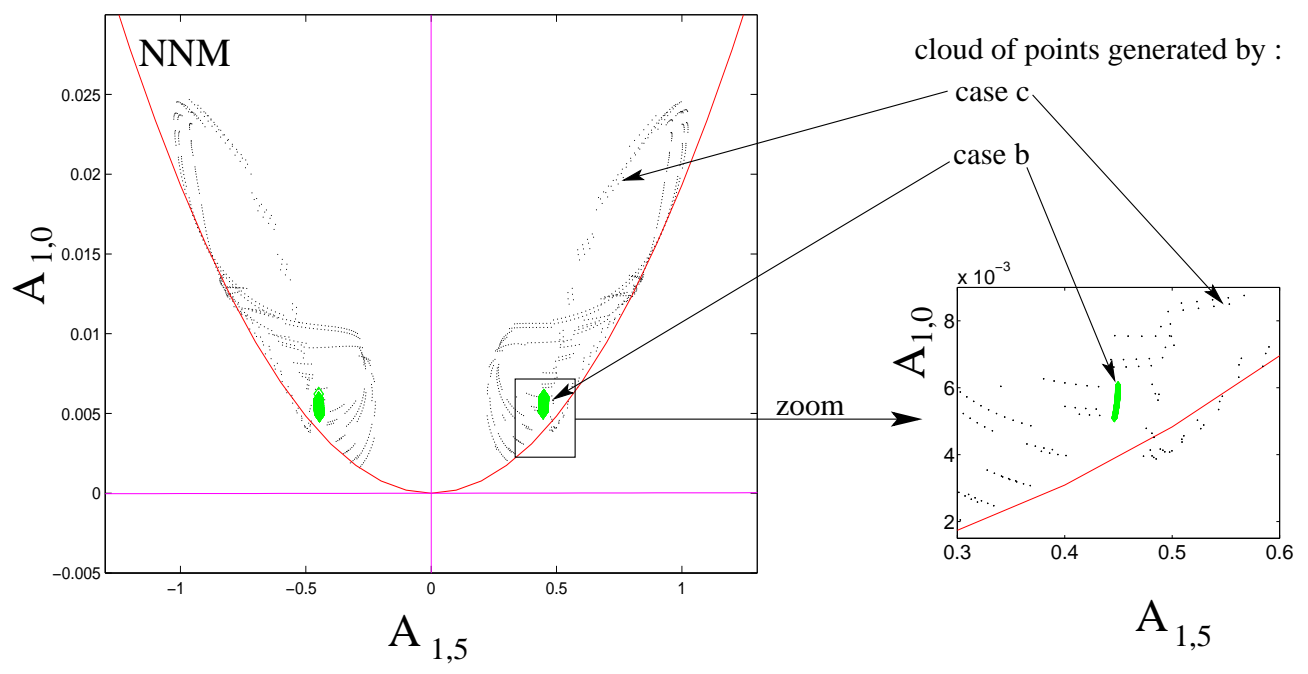

Figure 33: Poincaré section in $\left(A_{(1,5)}, A_{(1,0)}\right)$. Cloud of points: section of the orbits with the Poincaré plane, cases $b$ and $c$. POD differs very little from the original axis, whereas the cut of the 4-dimensional invariant manifold (curved hyperbolic line) goes right in-between the points (NNMs).

of $A_{(1,0)}$, though non-resonant, is not negligible, as ascertained by Figs. 31(e) and 33. The generating axis of the POD and NNM-based ROMS are also shown in Fig. 33. As already underlined, the POMs differ very slightly from the linear modes. On the other hand, the NNM method allows, thanks to normal form theory, to include the non-resonant coupling terms into the change of coordinates. The cut of the NNM is shown in Fig. 33, one can see that it takes naturally into account this non-resonant coupling term into the geometry of the manifold, so that the subspaces are very near the points of the reference simulation. This explains why the POD method need to take into account the third POM: otherwise the axisymmetric coupling would have been missed, hence resulting in an incorrect ROM. On the other hand, The NNM-based ROM is accurate with two NNMs only, as the bending of the phase space is taken into account in the nonlinear change of coordinates.

As a conclusion on this section, the examples with shell models have shown the ability of the NNMbased ROMs to produce accurate models for predicting the resonant forced response of thin structures with moderately large vibration amplitudes. The last subsection where comparison with the POD method has been drawn out, underlined two important features of the NNM method:

- the method is directly applicable on the resulting dynamical system, as a supplementary (nonlinear) change of coordinates. There is no need to compute, as in the POD method, a reference solution, in order to be able to build the ROM. This is a great advantage, more especially as the choice of correct solutions is a tricky problem that need experience and cannot be realized blindly, see e.g. $[6]$.

- thanks to the invariant (curved) based span of the phase space, the results obtained are of better agreement, with less NNMs than POMs. This is the advantage of using a representation basis that have a physical meaning, and is able to properly catch the non-resonant couplings, that are important for the geometry, but not for the dynamics. This underlines again a key idea claiming for using NNMS for reduced-order modeling. There is an "apparent" complexity of the dynamics which is only due to the non-resonant coupling terms, which creates the curvature of the invariant manifolds. This complexity is said to be "apparent" since it can be cancelled through a nonlinear change of coordinates. Once this complexity embedded in the geometry, a better reduction is 
obtained, and the complexity due to the resonant terms -that are really key for the dynamical properties of the solutions- can be more easily studied.

These conclusions need however to be moderated by the two following points:

- As proposed, the NNM-based ROM relies on two important assumptions: a third-order asymptotic method is used, and a time-invariant manifold is adopted. These assumptions have the great advantage to produce simple ROMS that are derived quickly without complicated numerical computations. However, increasing the amplitudes of vibration, the NNM-based ROM can lose its accuracy due to these two assumptions.

- The NNM method relies on a local theory, whereas the POD method is global (in the sense of the phase space). Hence the POD method can be more robust to large variations of the parameters. This is for example illustrated in [8].

\section{References}

[1] M. Amabili. Theory and experiments for large-amplitude vibrations of empty and fluid-filled circular cylindrical shell with imperfections. Journal of Sound and Vibration, 262(4):921-975, 2003.

[2] M. Amabili. Non-linear vibrations of doubly-curved shallow shells. International Journal of Nonlinear Mechanics, 40(5):683-710, 2005.

[3] M. Amabili and M. P. Païdoussis. Review of studies on geometrically nonlinear vibrations and dynamics of circular cylindrical shells and panels, with and without fluid-structure interaction. ASME Applied Mechanical Review, 56(4):349-381, 2003.

[4] M. Amabili, F. Pellicano, and M. P. Païdoussis. Non-linear vibrations of simply supported, circular cylindrical shells, coupled to quiescent fluid. Journal of Fluids and Structures, 12:883-918, 1998.

[5] M. Amabili, F. Pellicano, and M. P. Païdoussis. Non-linear dynamics and stability of circular cylindrical shells containing flowing fluid, part II: large-amplitude vibrations without flow. Journal of Sound and Vibration, 228(5):1103-1124, 1999.

[6] M. Amabili, A. Sarkar, and M. P. Païdoussis. Reduced-order models for nonlinear vibrations of cylindrical shells via the proper orthogonal decomposition method. Journal of Fluids and Structures, 18(2):227-250, 2003.

[7] M. Amabili, A. Sarkar, and M. P. Païdoussis. Chaotic vibrations of circular cylindrical shells: Galerkin versus reduced-order models via the proper orthogonal decomposition method. Journal of Sound and Vibration, 290(3-5):736-762, 2006.

[8] M. Amabili and C. Touzé. Reduced-order models for non-linear vibrations of fluid-filled circular cylindrical shells: comparison of pod and asymptotic non-linear normal modes methods. Journal of Fluids and Structures, 23(6):885-903, 2007.

[9] G. Berkooz, P. Holmes, and J.L. Lumley. The proper orthogonal decomposition in the analysis of turbulent flows. Annual review of Fluid Mechanics, 25:539-575, 1993.

[10] F. Blanc, C. Touzé, J.-F. Mercier, and A.-S. Bonnet Ben-Dhia. On the numerical computation of nonlinear normal modes for reduced-order modelling of conservative vibratory systems. Mechanical Systems and Signal Processing, submitted, 2012.

[11] H-N Chu and G. Herrmann. Influence of large amplitudes on free flexural vibrations of rectangular elastic plates. Journal of Applied Mechanics, 23:532-540, 1956. 
[12] E.J. Doedel, R. Paffenroth, A.R. Champneys, T.F. Fairgrieve, Y.A. Kuznetsov, B.E. Oldeman, B. Sandstede, and X. Wang. Auto 2000: Continuation and bifurcation software for ordinary differential equations. Technical report, Concordia University, 2002.

[13] H. Dulac. Solutions d'un système d'équations différentielles dans le voisinage de valeurs singulières. Bulletin de la Société Mathématique de France, 40:324-383, 1912.

[14] G. J. Efstathiades. A new approach to the large-deflection vibrations of imperfect circular disks using Galerkin's procedure. Journal of Sound and Vibration, 16(2):231-253, 1971.

[15] C. Elphick, G. Iooss, and E. Tirapegui. Normal form reduction for time-periodically driven differential equations. Physics Letters A, 120(9):459-463, 1987.

[16] H.A. Evensen and R.M. Ewan-Iwanowsky. Dynamic response and stability of shallow spherical shells subject to time-dependent loading. AIAA Journal, 5(5):969-976, 1967.

[17] B.F. Feeny and R. Kappagantu. On the physical interpretation of proper orthogonal modes in vibrations. Journal of Sound and Vibration, 211(4):607-616, 1997.

[18] P. B. Gonçalves. Axisymmetric vibrations of imperfect shallow spherical caps under pressure loading. Journal of Sound and Vibration, 174(2):249-260, 1994.

[19] P. L. Grossman, B. Koplik, and Y-Y. Yu. Nonlinear vibrations of shallow spherical shells. ASME Journal of Applied Mechanics, 39E:451-458, 1969.

[20] J. Guckenheimer and P. Holmes. Nonlinear oscillations, dynamical systems and bifurcations of vector fields. Springer-Verlag, New-York, 1983.

[21] G. Iooss and M. Adelmeyer. Topics in bifurcation theory. World scientific, New-York, 1998. second edition.

[22] L. Jézéquel and C. H. Lamarque. Analysis of non-linear dynamical systems by the normal form theory. Journal of Sound and Vibration, 149(3):429-459, 1991.

[23] D. Jiang. Nonlinear modal analysis based on invariant manifolds. Application to rotating blade systems. PhD thesis, University of Michigan, 2004.

[24] D. Jiang, C. Pierre, and S. Shaw. Nonlinear normal modes for vibratory systems under harmonic excitation. Journal of Sound and Vibration, 288(4-5):791-812, 2005.

[25] Th.von Kármán. Festigkeitsprobleme im maschinenbau. Encyklopdie der Mathematischen Wissenschaften, 4(4):311-385, 1910.

[26] G. Kerschen and J.C. Golinval. Physical interpretation of the proper orthogonal modes using the singular value decomposition. Journal of Sound and Vibration, 249(5):849-865, 2002.

[27] G. Kerschen, J.C. Golinval, A.F. Vakakis, and L.A. Bergman. The method of proper orthogonal decomposition for dynamical characterization and order reduction of mechanical systems: an overview. Nonlinear Dynamics, 41:147-169, 2005.

[28] C.-H. Lamarque, C. Touzé, and O. Thomas. An upper bound for validity limits of asymptotic analytical approaches based on normal form theory. Nonlinear Dynamics, submitted, 2012.

[29] P. Manneville. Dissipative structures and weak turbulence. Academic Press, 1990.

[30] P. Manneville. Instabilités, chaos et turbulence. Editions de l'Ecole Polytechnique, Palaiseau, 2004. 
[31] A. H. Nayfeh. On direct methods for constructing nonlinear normal modes of continuous systems. Journal of Vibration and Control, 1:389-430, 1995.

[32] A. H. Nayfeh and W. Lacarbonara. On the discretization of distributed-parameter systems with quadratic and cubic non-linearities. Nonlinear Dynamics, 13:203-220, 1997.

[33] A. H. Nayfeh, J. F. Nayfeh, and D. T. Mook. On methods for continuous systems with quadratic and cubic nonlinearities. Nonlinear Dynamics, 3:145-162, 1992.

[34] A. H. Nayfeh and S. A. Nayfeh. On nonlinear normal modes of continuous systems. Trans. ASME/Journal of Vibration and Acoustics, 116:129-136, 1994.

[35] K. A. V. Pandalai and M. Sathyamoorthy. On the modal equations of large amplitude flexural vibration of beams, plates, rings and shells. International Journal of Non-linear Mechanics, 8(3):213$218,1973$.

[36] F. Pellicano, M. Amabili, and M. P. Païdoussis. Effect of the geometry on the non-linear vibration of circular cylindrical shells. International Journal of Non-linear Mechanics, 37:1181-1198, 2002.

[37] E. Pesheck. Reduced-order modeling of nonlinear structural systems using nonlinear normal modes and invariant manifolds. PhD thesis, University of Michigan, 2000.

[38] E. Pesheck and C. Pierre. A global methodology for the modal reduction of large non-linear systems containing quadratic and cubic non-linearities. In Proc. of ASME DETC'97, 1997.

[39] H. Poincaré. Les méthodes nouvelles de la mécanique céleste. Gauthiers-Villars, Paris, 1892.

[40] A. Sarkar and M. P. Païdoussis. A cantilever conveying fluid: coherent modes versus beam modes. International Journal of Non-Linear Mechanics, 39:467481, 2004.

[41] M. Sathyamoorthy. Vibrations of moderatly thick shallow spherical shells at large amplitude. Journal of Sound and Vibration, 172(1):63-70, 1994.

[42] S. W. Shaw and C. Pierre. Non-linear normal modes and invariant manifolds. Journal of Sound and Vibration, 150(1):170-173, 1991.

[43] S. W. Shaw and C. Pierre. Normal modes for non-linear vibratory systems. Journal of Sound and Vibration, 164(1):85-124, 1993.

[44] S. Sridhar, D. T. Mook, and A. H. Nayfeh. Non-linear resonances in the forced responses of plates, part I: symmetric responses of circular plates. Journal of Sound and Vibration, 41(3):359-373, 1975.

[45] O. Thomas and S. Bilbao. Geometrically nonlinear flexural vibrations of plates: In-plane boundary conditions and some symmetry properties. Journal of Sound and Vibration, 315(3):569-590, 2008.

[46] O. Thomas, C. Touzé, and A. Chaigne. Asymmetric non-linear forced vibrations of free-edge circular plates, part II: experiments. Journal of Sound and Vibration, 265(5):1075-1101, 2003.

[47] O. Thomas, C. Touzé, and A. Chaigne. Non-linear vibrations of free-edge thin spherical shells: modal interaction rules and 1:1:2 internal resonance. International Journal of Solids and Structures, 42(11-12):3339-3373, 2005.

[48] S. A. Tobias. Free undamped non-linear vibrations of imperfect circular disks. Proc. of the Instn. of Mech. Eng., 171:691-700, 1957. 
[49] C. Touzé. A normal form approach for non-linear normal modes. Technical report, Publications du LMA, numéro 156, (ISSN: 1159-0947, ISBN: 2-909669-20-3), 2003.

[50] C. Touzé and M. Amabili. Non-linear normal modes for damped geometrically non-linear systems: application to reduced-order modeling of harmonically forced structures. Journal of Sound and Vibration, 298(4-5):958-981, 2006.

[51] C. Touzé, M. Amabili, and O. Thomas. Reduced-order models for large-amplitude vibrations of shells including in-plane inertia. Computer Methods in Applied Mechanics and Engineering, 197(21-24):2030-2045, 2008.

[52] C. Touzé, C. Camier, G. Favraud, and O. Thomas. Effect of imperfections and damping on the type of non-linearity of circular plates and shallow spherical shells. Mathematical Problems in Engineering, 2008:Article ID 678307, 2008. doi:10.1155/2008/678307.

[53] C. Touzé and O. Thomas. Non-linear behaviour of free-edge shallow spherical shells: effect of the geometry. International Journal of Non-linear Mechanics, 41(5):678-692, 2006.

[54] C. Touzé, O. Thomas, and A. Chaigne. Asymmetric non-linear forced vibrations of free-edge circular plates, part I: theory. Journal of Sound and Vibration, 258(4):649-676, 2002.

[55] C. Touzé, O. Thomas, and A. Chaigne. Hardening/softening behaviour in non-linear oscillations of structural systems using non-linear normal modes. Journal of Sound and Vibration, 273(1-2):77$101,2004$.

[56] T. K. Varadan and K. A. V. Pandalai. Non-linear flexural oscillations of orthotropic shallow spherical shells. Computers and Structures, 9:417-425, 1978.

[57] S. Wiggins. Introduction to applied nonlinear dynamical systems and chaos. Springer-Verlag, New-York, 2003. Second edition.

[58] N. Yamaki. Influence of large amplitudes on flexural vibrations of elastic plates. ZAMM, 41(12):501-510, 1961.

[59] K. Yasuda and G. Kushida. Nonlinear forced oscillations of a shallow spherical shell. Bull. JSME, 27(232):2233-2240, 1984. 


\section{A Expressions of the coefficients for the normal transform in the conser- vative case}

Expressions of the coefficients $\left(a_{i j}^{p}, b_{i j}^{p}, r_{i j k}^{p}, u_{i j k}^{p}, \gamma_{i j}^{p}, \mu_{i j k}^{p}, \nu_{i j k}^{p}\right)$ used in Eqs. (39), which allows one to go from the modal variables (associated with the orthogonal linear grid) to the normal coordinates (associated to the invariant manifold and the curved grid) are given here.

\section{A.1 Quadratic coefficients}

- The following expressions are obtained, $\forall p=1, \ldots, N$ :

$$
\begin{aligned}
\forall i=1, \ldots, N, \forall j & \geq i, \ldots, N: \\
a_{i j}^{p} & =\frac{\omega_{i}^{2}+\omega_{j}^{2}-\omega_{p}^{2}}{D_{i j p}} g_{i j}^{p} \\
b_{i j}^{p} & =\frac{2}{D_{i j p}} g_{i j}^{p} \\
c_{i j}^{p} & =0 \\
\alpha_{i j}^{p} & =0 \\
\beta_{i j}^{p} & =0 \\
\gamma_{i i}^{p} & =\frac{2}{4 \omega_{i}^{2}-\omega_{p}^{2}} g_{i i}^{p} \\
\forall i=1, \ldots, N, \forall j & >i, \ldots, N: \\
\gamma_{i j}^{p} & =\frac{\omega_{j}^{2}-\omega_{i}^{2}-\omega_{p}^{2}}{D_{i j p}} g_{i j}^{p} \\
\gamma_{j i}^{p} & =\frac{\omega_{i}^{2}-\omega_{j}^{2}-\omega_{p}^{2}}{D_{i j p}} g_{i j}^{p}
\end{aligned}
$$

where $D_{i j p}=\left(\omega_{i}+\omega_{j}-\omega_{p}\right)\left(\omega_{i}+\omega_{j}+\omega_{p}\right)\left(-\omega_{j}+\omega_{i}+\omega_{p}\right)\left(\omega_{i}-\omega_{j}-\omega_{p}\right)$.

\section{A.2 Cubic coefficients}

- The following coefficients, which correspond to the trivially resonant terms, are equal to zero:

$$
\begin{gathered}
\forall p=1, \ldots, N: \quad u_{p p p}^{p}=r_{p p p}^{p}=\mu_{p p p}^{p}=\nu_{p p p}^{p}=0 \\
\forall j>p \ldots N: r_{p j j}^{p}=u_{p j j}^{p}=u_{j p j}^{p}=0 \\
\mu_{p j j}^{p}=\nu_{p j j}^{p}=\nu_{j p j}^{p}=0 \\
\forall i<p: \quad r_{i i p}^{p}=u_{i i p}^{p}=u_{p i i}^{p}=0 \\
\mu_{i i p}^{p}=\nu_{i i p}^{p}=\nu_{p i i}^{p}=0
\end{gathered}
$$


- The non-zero coefficients are now given, $\forall p=1, \ldots, N$ :

$\forall i=1, \ldots, N, i \neq p$ :

$$
\begin{aligned}
r_{i i i}^{p} & =\frac{1}{D_{i p}^{(1)}}\left[\left(7 \omega_{i}^{2}-\omega_{p}^{2}\right)\left(h_{i i i}^{p}+A_{i i i}^{p}\right)+2 \omega_{i}^{4} B_{i i i}^{p}\right] \\
u_{i i i}^{p} & =\frac{1}{D_{i p}^{(1)}}\left[6 h_{i i i}^{p}+6 A_{i i i}^{p}+\left(3 \omega_{i}^{2}-\omega_{p}^{2}\right) B_{i i i}^{p}\right] \\
\mu_{i i i}^{p} & =u_{i i i}^{p} \\
\nu_{i i i}^{p} & =\frac{1}{D_{i p}^{(1)}}\left[\left(9 \omega_{i}^{2}-3 \omega_{p}^{2}\right)\left(h_{i i i}^{p}+A_{i i i}^{p}\right)+2 \omega_{p}^{2} \omega_{i}^{2} B_{i i i}^{p}\right]
\end{aligned}
$$

where $D_{i p}^{(1)}=\left(\omega_{p}^{2}-\omega_{i}^{2}\right)\left(\omega_{p}^{2}-9 \omega_{i}^{2}\right)$.

$$
\begin{aligned}
& \forall i=1, \ldots, N-1, i \neq p, \forall j>i, \ldots, N \text { : } \\
& r_{i j j}^{p}=\frac{\omega_{i}^{2}\left(\omega_{i}^{2}-2 \omega_{j}^{2}-2 \omega_{p}^{2}\right)+\left(\omega_{p}^{2}-4 \omega_{j}^{2}\right)\left(\omega_{p}^{2}-2 \omega_{j}^{2}\right)}{\left(\omega_{i}^{2}-\omega_{p}^{2}\right) D_{i j p}^{(1)}}\left[A_{i j j}^{p}+A_{j i j}^{p}+h_{i j j}^{p}\right] \\
& -\frac{2 \omega_{j}^{4}\left(\omega_{p}^{2}-4 \omega_{j}^{2}+3 \omega_{i}^{2}\right)}{\left(\omega_{i}^{2}-\omega_{p}^{2}\right) D_{i j p}^{(1)}} B_{i j j}^{p}+\frac{2 \omega_{i}^{2} \omega_{j}^{2}}{D_{i j p}^{(1)}} B_{j i j}^{p} \\
& u_{i j j}^{p}=\frac{\omega_{i}^{2}\left(\omega_{i}^{2}-2 \omega_{j}^{2}-2 \omega_{p}^{2}\right)+\left(\omega_{p}^{2}-4 \omega_{j}^{2}\right)\left(\omega_{p}^{2}-2 \omega_{j}^{2}\right)}{\left(\omega_{i}^{2}-\omega_{p}^{2}\right) D_{i j p}^{(1)}} B_{i j j}^{p} \\
& +\frac{8 \omega_{j}^{2}-6 \omega_{i}^{2}-2 \omega_{p}^{2}}{\left(\omega_{i}^{2}-\omega_{p}^{2}\right) D_{i j p}^{(1)}}\left[A_{i j j}^{p}+A_{j i j}^{p}+h_{i j j}\right]-\frac{2 \omega_{i}^{2}}{D_{i j p}^{(1)}} B_{j i j}^{p} \\
& u_{j i j}^{p}=\frac{1}{D_{i j p}^{(1)}}\left[4\left(A_{j i j}^{p}+A_{i j j}^{p}+h_{i j j}^{p}\right)-4 w_{j}{ }^{2} B_{i j j}^{p}+\left(4 w_{j}{ }^{2}-w_{p}{ }^{2}+w_{i}{ }^{2}\right) B_{j i j}^{p}\right] \\
& \nu_{i j j}^{p}=\frac{6 \omega_{i}^{2} \omega_{j}^{2}+2 \omega_{j}^{2} \omega_{p}^{2}+2 \omega_{p}^{2} \omega_{i}^{2}-8 \omega_{j}^{4}-\omega_{p}^{4}-\omega_{i}^{4}}{\left(\omega_{p}^{2}-\omega_{i}^{2}\right) D_{i j p}^{(1)}}\left[A_{i j j}^{p}+A_{j i j}^{p}+h_{i j j}^{p}\right] \\
& +\frac{2 \omega_{j}^{4}\left(3 \omega_{p}^{2}+\omega_{i}^{2}-4 \omega_{j}^{2}\right)}{\left(\omega_{p}^{2}-\omega_{i}^{2}\right) D_{i j p}^{(1)}} B_{i j j}^{p}-\frac{\omega_{j}^{2}\left(-\omega_{p}^{2}+4 \omega_{j}^{2}-\omega_{i}^{2}\right)}{D_{i j p}^{(1)}} B_{j i j}^{p} \\
& \nu_{j i j}^{p}=\frac{8 \omega_{j}^{2}-2 \omega_{i}^{2}-2 \omega_{p}^{2}}{D_{i j p}^{(1)}}\left[A_{i j j}^{p}+A_{j i j}^{p}+h_{i j j}^{p}\right] \\
& +\frac{2 \omega_{j}^{2} \omega_{p}^{2}-8 \omega_{j}^{4}+2 \omega_{j}^{2} \omega_{i}^{2}}{D_{i j p}^{(1)}} B_{i j j}^{p}+\frac{\omega_{p}^{2} \omega_{i}^{2}+4 \omega_{j}^{2} \omega_{i}^{2}-\omega_{i}^{4}}{D_{i j p}^{(1)}} B_{j i j}^{p} \\
& \mu_{i j j}^{p}=\frac{6 \omega_{i}^{2} \omega_{j}^{2}+2 \omega_{j}^{2} \omega_{p}^{2}+2 \omega_{p}^{2} \omega_{i}^{2}-8 \omega_{j}^{4}-\omega_{p}^{4}-\omega_{i}^{4}}{\left(\omega_{p}^{2}-\omega_{i}^{2}\right) D_{i j p}^{(1)}} B_{i j j}^{p} \\
& +\frac{6 \omega_{p}^{2}+2 \omega_{i}^{2}-8 \omega_{j}^{2}}{\left(\omega_{p}^{2}-\omega_{i}^{2}\right) D_{i j p}^{(1)}}\left[A_{i j j}^{p}+A_{j i j}^{p}+h_{i j j}^{p}\right]+\frac{4 \omega_{j}^{2}-\omega_{i}^{2}-\omega_{p}^{2}}{D_{i j p}^{(1)}} B_{j i j}^{p}
\end{aligned}
$$

where $D_{i j p}^{(1)}=\left(\omega_{p}+\omega_{i}-2 \omega_{j}\right)\left(\omega_{p}+\omega_{i}+2 \omega_{j}\right)\left(-\omega_{p}+\omega_{i}+2 \omega_{j}\right)\left(-\omega_{p}+\omega_{i}-2 \omega_{j}\right)$. 


$$
\begin{aligned}
& \forall i=1, \ldots, N-1, \forall j>i, \ldots, N, j \neq p: \\
& r_{i i j}^{p}=\frac{2 \omega_{i}^{2}\left(4 \omega_{i}^{2}-3 \omega_{p}^{2}-\omega_{j}^{2}\right)+\left(\omega_{p}-\omega_{j}\right)^{2}\left(\omega_{p}+\omega_{j}\right)^{2}}{\left(\omega_{p}^{2}-\omega_{j}^{2}\right) D_{i j p}^{(2)}}\left[-A_{i i j}^{p}-A_{j i i}^{p}-h_{i i j}^{p}\right] \\
& -\frac{2 \omega_{i}^{4}\left(4 \omega_{i}^{2}-\omega_{p}^{2}-3 \omega_{j}^{2}\right)}{\left(\omega_{p}^{2}-\omega_{j}^{2}\right) D_{i j p}^{(2)}} B_{j i i}^{p}-\frac{2 \omega_{i}^{2} \omega_{j}^{2}}{D_{i j p}^{(2)}} B_{i i j}^{p} \\
& u_{j i i}^{p}=-\frac{2 \omega_{i}^{2}\left(4 \omega_{i}^{2}-3 \omega_{p}^{2}-\omega_{j}^{2}\right)+\left(\omega_{p}-\omega_{j}\right)^{2}\left(\omega_{p}+\omega_{j}\right)^{2}}{\left(\omega_{p}^{2}-\omega_{j}^{2}\right) D_{i j p}^{(2)}} B_{j i i}^{p} \\
& +\frac{8 \omega_{i}^{2}-2 \omega_{p}^{2}-6 \omega_{j}^{2}}{\left(\omega_{p}^{2}-\omega_{j}^{2}\right) D_{i j p}^{(2)}}\left[-A_{j i i}^{p}-A_{i i j}^{p}-h_{i i j}^{p}\right]-\frac{2 \omega_{j}^{2}}{D_{i j p}^{(2)}} B_{i i j}^{p} \\
& u_{i i j}^{p}=\frac{1}{D_{i j p}^{(2)}}\left[4\left(A_{j i i}^{p}+A_{i i j}^{p}+h_{i i j}^{p}\right)-4 \omega_{i}^{2} B_{j i i}^{p}-\left(\omega_{p}^{2}-\omega_{j}^{2}-4 \omega_{i}^{2}\right) B_{i i j}^{p}\right] \\
& \nu_{j i i}^{p}=\frac{8 \omega_{i}^{4}+\omega_{j}^{4}+\omega_{p}^{4}-2 \omega_{p}^{2} \omega_{i}^{2}-6 \omega_{i}^{2} \omega_{j}^{2}-2 \omega_{j}^{2} \omega_{p}^{2}}{\left(\omega_{j}^{2}-\omega_{p}^{2}\right) D_{i j p}^{(2)}}\left[A_{j i i}^{p}+A_{i i j}^{p}+h_{i i j}^{p}\right] \\
& +\frac{8 \omega_{i}^{6}-2 \omega_{j}^{2} \omega_{i}^{4}-6 \omega_{p}^{2} \omega_{i}^{4}}{\left(\omega_{j}^{2}-\omega_{p}^{2}\right) D_{i j p}^{(2)}} B_{j i i}^{p}+\frac{\omega_{i}^{2}\left(\omega_{j}^{2}-4 \omega_{i}^{2}+\omega_{p}^{2}\right)}{D_{i j p}^{(2)}} B_{i i j}^{p} \\
& \nu_{i i j}^{p}=\frac{8 \omega_{i}^{2}-2 \omega_{p}^{2}-2 \omega_{j}^{2}}{D_{i j p}^{(2)}}\left[A_{j i i}^{p}+A_{i i j}^{p}+h_{i i j}^{p}\right] \\
& +\frac{2 \omega_{i}^{2} \omega_{j}^{2}-8 \omega_{i}^{4}+2 \omega_{i}^{2} \omega_{p}^{2}}{D_{i j p}^{(2)}} B_{j i i}^{p}+\frac{-\omega_{j}^{4}+4 \omega_{i}^{2} \omega_{j}^{2}+\omega_{j}^{2} \omega_{p}^{2}}{D_{i j p}^{(2)}} B_{i i j}^{p} \\
& \mu_{i i j}^{p}=\frac{8 \omega_{i}^{2}-2 \omega_{j}^{2}-6 \omega_{p}^{2}}{\left(\omega_{j}^{2}-\omega_{p}^{2}\right) D_{i j p}^{(2)}}\left[A_{i i j}^{p}+A_{j i i}^{p}+h_{i i j}^{p}\right]+\frac{4 \omega_{i}^{2}-\omega_{j}^{2}-\omega_{p}^{2}}{D_{i j p}^{(2)}} B_{i i j}^{p} \\
& -\frac{6 \omega_{i}^{2} \omega_{j}^{2}+2 \omega_{j}^{2} \omega_{p}^{2}+2 \omega_{i}^{2} \omega_{p}^{2}-8 \omega_{i}^{4}-\omega_{p}^{4}-\omega_{j}^{4}}{\left(\omega_{j}^{2}-\omega_{p}^{2}\right) D_{i j p}^{(2)}} B_{j i i}^{p}
\end{aligned}
$$

where $D_{i j p}^{(2)}=\left(\omega_{p}+2 \omega_{i}-\omega_{j}\right)\left(\omega_{p}+2 \omega_{i}+\omega_{j}\right)\left(-\omega_{p}+2 \omega_{i}+\omega_{j}\right)\left(-\omega_{p}+2 \omega_{i}-\omega_{j}\right)$.

$$
\begin{aligned}
& \forall i=1, \ldots, N-2, \forall j>i, \ldots, N-1, \forall k>j, \ldots, N: \\
& u_{i j k}^{p}=\frac{1}{D_{i j k p}^{(3)}}\left[\quad \left(2 \omega_{j}^{4}-6 \omega_{i}^{4}-4 \omega_{k}^{2} \omega_{p}^{2}+4 \omega_{i}^{2} \omega_{p}^{2}+4 \omega_{i}^{2} \omega_{k}^{2}+2 \omega_{k}^{4}\right.\right. \\
& \left.+4 \omega_{i}^{2} \omega_{j}^{2}+2 \omega_{p}^{4}-4 \omega_{k}^{2} \omega_{j}^{2}-4 \omega_{j}^{2} \omega_{p}^{2}\right)\left(h_{i j k}^{p}+A_{i j k}^{p}+A_{k i j}^{p}+A_{j i k}^{p}\right) \\
& +\left(\quad-\omega_{k}^{2} \omega_{j}^{4}-\omega_{i}^{2} \omega_{k}^{4}-2 \omega_{j}^{2} \omega_{i}^{2} \omega_{p}^{2}+3 \omega_{j}^{2} \omega_{p}^{4}-\omega_{k}^{4} \omega_{j}^{2}-2 \omega_{i}^{2} \omega_{k}^{2} \omega_{p}^{2}\right. \\
& +3 \omega_{i}^{2} \omega_{p}^{4}-\omega_{j}^{4} \omega_{i}^{2}-\omega_{i}^{4} \omega_{k}^{2}+3 \omega_{k}^{2} \omega_{p}^{4}+\omega_{i}^{6}-\omega_{p}^{6}-3 \omega_{k}^{4} \omega_{p}^{2}+\omega_{k}^{6}-3 \omega_{j}^{4} \omega_{p}^{2} \\
& \left.+10 \omega_{i}^{2} \omega_{k}^{2} \omega_{j}^{2}+\omega_{j}^{6}-2 \omega_{k}^{2} \omega_{j}^{2} \omega_{p}^{2}-3 \omega_{i}^{4} \omega_{p}^{2}-\omega_{j}^{2} \omega_{i}^{4}\right) B_{i j k}^{p} \\
& +\left(\quad-2 \omega_{j}^{4} \omega_{i}^{2}+4 \omega_{i}^{4} \omega_{p}^{2}+4 \omega_{j}^{2} \omega_{i}^{4}+6 \omega_{i}^{2} \omega_{k}^{4}-4 \omega_{i}^{4} \omega_{k}^{2}\right. \\
& \left.+4 \omega_{j}^{2} \omega_{i}^{2} \omega_{p}^{2}-2 \omega_{i}^{2} \omega_{p}^{4}-2 \omega_{i}^{6}-4 \omega_{i}^{2} \omega_{k}^{2} \omega_{p}^{2}-4 \omega_{i}^{2} \omega_{k}^{2} \omega_{j}^{2}\right) B_{j i k}^{p} \\
& +\left(-2 \omega_{i}^{2} \omega_{p}^{4}+6 \omega_{j}^{4} \omega_{i}^{2}+4 \omega_{i}^{2} \omega_{k}^{2} \omega_{p}^{2}-4 \omega_{i}^{2} \omega_{k}^{2} \omega_{j}^{2}-4 \omega_{j}^{2} \omega_{i}^{2} \omega_{p}^{2}\right. \\
& \left.\left.-4 \omega_{j}^{2} \omega_{i}^{4}-2 \omega_{i}^{2} \omega_{k}^{4}+4 \omega_{i}^{4} \omega_{p}^{2}-2 \omega_{i}^{6}+4 \omega_{i}^{4} \omega_{k}^{2}\right) B_{k i j}^{p}\right]
\end{aligned}
$$




$$
\begin{aligned}
& u_{j i k}^{p}=\frac{1}{D_{i j k p}^{(3)}}\left[\quad \left(-2 \omega_{p}^{4}+6 \omega_{j}^{4}-2 \omega_{i}^{4}-4 \omega_{k}^{2} \omega_{j}^{2}+4 \omega_{k}^{2} \omega_{p}^{2}\right.\right. \\
& \left.-2 \omega_{k}^{4}+4 \omega_{i}^{2} \omega_{k}^{2}-4 \omega_{i}^{2} \omega_{j}^{2}+4 \omega_{i}^{2} \omega_{p}^{2}-4 \omega_{j}^{2} \omega_{p}^{2}\right)\left(h_{i j k}^{p}+A_{i j k}^{p}+A_{k i j}^{p}+A_{j i k}^{p}\right) \\
& +\left(2 \omega_{j}^{6}+4 \omega_{k}^{2} \omega_{j}^{2} \omega_{p}^{2}+4 \omega_{i}^{2} \omega_{k}^{2} \omega_{j}^{2}+2 \omega_{j}^{2} \omega_{i}^{4}+4 \omega_{k}^{2} \omega_{j}^{4}\right. \\
& \left.-4 \omega_{j}^{2} \omega_{i}^{2} \omega_{p}^{2}+2 \omega_{j}^{2} \omega_{p}^{4}-4 \omega_{j}^{4} \omega_{i}^{2}-4 \omega_{j}^{4} \omega_{p}^{2}-6 \omega_{k}^{4} \omega_{j}^{2}\right) B_{i j k}^{p} \\
& +\left(3 \omega_{j}^{4} \omega_{p}^{2}+\omega_{k}^{2} \omega_{j}^{4}-3 \omega_{k}^{2} \omega_{p}^{4}+\omega_{i}^{2} \omega_{k}^{4}+\omega_{k}^{4} \omega_{j}^{2}+3 \omega_{i}^{4} \omega_{p}^{2}\right. \\
& +2 \omega_{i}^{2} \omega_{k}^{2} \omega_{p}^{2}+\omega_{j}^{4} \omega_{i}^{2}+\omega_{i}^{4} \omega_{k}^{2}-\omega_{i}^{6}+3 \omega_{k}^{4} \omega_{p}^{2}-\omega_{j}^{6}-\omega_{k}^{6}+2 \omega_{j}^{2} \omega_{i}^{2} \omega_{p}^{2}+\omega_{p}^{6} \\
& \left.+2 \omega_{k}^{2} \omega_{j}^{2} \omega_{p}^{2}-3 \omega_{j}^{2} \omega_{p}^{4}-10 \omega_{i}^{2} \omega_{k}^{2} \omega_{j}^{2}-3 \omega_{i}^{2} \omega_{p}^{4}+\omega_{j}^{2} \omega_{i}^{4}\right) B_{j i k}^{p} \\
& +\left(4 \omega_{i}^{2} \omega_{k}^{2} \omega_{j}^{2}+4 \omega_{j}^{4} \omega_{i}^{2}+2 \omega_{j}^{2} \omega_{p}^{4}-6 \omega_{j}^{2} \omega_{i}^{4}+2 \omega_{k}^{4} \omega_{j}^{2}-4 \omega_{j}^{4} \omega_{p}^{2}\right. \\
& \left.\left.+2 \omega_{j}^{6}-4 \omega_{k}^{2} \omega_{j}^{4}-4 \omega_{k}^{2} \omega_{j}^{2} \omega_{p}^{2}+4 \omega_{j}^{2} \omega_{i}^{2} \omega_{p}^{2}\right) B_{k i j}^{p}\right] \\
& u_{k i j}^{p}=\frac{1}{D_{i j k p}^{(3)}}\left[\left(2 \omega_{p}^{4}+2 \omega_{j}^{4}+4 \omega_{i}^{2} \omega_{k}^{2}-6 \omega_{k}^{4}+4 \omega_{k}^{2} \omega_{p}^{2}-4 \omega_{i}^{2} \omega_{p}^{2}\right.\right. \\
& \left.+4 \omega_{k}^{2} \omega_{j}^{2}-4 \omega_{i}^{2} \omega_{j}^{2}-4 \omega_{j}^{2} \omega_{p}^{2}+2 \omega_{i}^{4}\right)\left(h_{i j k}^{p}+A_{i j k}^{p}+A_{k i j}^{p}+A_{j i k}^{p}\right) \\
& +\left(-4 \omega_{i}^{2} \omega_{k}^{2} \omega_{j}^{2}-2 \omega_{k}^{6}-2 \omega_{i}^{4} \omega_{k}^{2}-4 \omega_{k}^{2} \omega_{j}^{2} \omega_{p}^{2}+4 \omega_{i}^{2} \omega_{k}^{2} \omega_{p}^{2}\right. \\
& \left.+6 \omega_{k}^{2} \omega_{j}^{4}-2 \omega_{k}^{2} \omega_{p}^{4}+4 \omega_{i}^{2} \omega_{k}^{4}-4 \omega_{k}^{4} \omega_{j}^{2}+4 \omega_{k}^{4} \omega_{p}^{2}\right) B_{i j k}^{p} \\
& +\left(\quad-2 \omega_{k}^{6}-4 \omega_{i}^{2} \omega_{k}^{4}+4 \omega_{k}^{2} \omega_{j}^{2} \omega_{p}^{2}-4 \omega_{i}^{2} \omega_{k}^{2} \omega_{p}^{2}-2 \omega_{k}^{2} \omega_{p}^{4}+6 \omega_{i}^{4} \omega_{k}^{2}\right. \\
& \left.-4 \omega_{i}^{2} \omega_{k}^{2} \omega_{j}^{2}+4 \omega_{k}^{4} \omega_{j}^{2}-2 \omega_{k}^{2} \omega_{j}^{4}+4 \omega_{k}^{4} \omega_{p}^{2}\right) B_{j i k}^{p} \\
& +\left(-\omega_{k}^{2} \omega_{j}^{4}-\omega_{i}^{2} \omega_{k}^{4}-2 \omega_{j}^{2} \omega_{i}^{2} \omega_{p}^{2}+3 \omega_{j}^{2} \omega_{p}^{4}-\omega_{k}^{4} \omega_{j}^{2}-2 \omega_{i}^{2} \omega_{k}^{2} \omega_{p}^{2}\right. \\
& +3 \omega_{i}^{2} \omega_{p}^{4}-\omega_{j}^{4} \omega_{i}^{2}-\omega_{i}^{4} \omega_{k}^{2}+3 \omega_{k}^{2} \omega_{p}^{4}+\omega_{i}^{6}-\omega_{p}^{6}-3 \omega_{k}^{4} \omega_{p}^{2}+\omega_{k}^{6} \\
& \left.\left.-3 \omega_{j}^{4} \omega_{p}^{2}+10 \omega_{i}^{2} \omega_{k}^{2} \omega_{j}^{2}+\omega_{j}^{6}-2 \omega_{k}^{2} \omega_{j}^{2} \omega_{p}^{2}-3 \omega_{i}^{4} \omega_{p}^{2}-\omega_{j}^{2} \omega_{i}^{4}\right) B_{k i j}^{p}\right] \\
& r_{i j k}^{p}=\frac{1}{D_{i j k p}^{(3)}}\left[\quad \left(-\omega_{k}^{2} \omega_{j}^{4}-\omega_{i}^{2} \omega_{k}^{4}-2 \omega_{j}^{2} \omega_{i}^{2} \omega_{p}^{2}+3 \omega_{j}^{2} \omega_{p}^{4}-\omega_{k}^{4} \omega_{j}^{2}\right.\right. \\
& -2 \omega_{i}^{2} \omega_{k}^{2} \omega_{p}^{2}+3 \omega_{i}^{2} \omega_{p}^{4}-\omega_{j}^{4} \omega_{i}^{2}-\omega_{i}^{4} \omega_{k}^{2}+3 \omega_{k}^{2} \omega_{p}^{4}+\omega_{i}^{6}-\omega_{p}^{6}-3 \omega_{k}^{4} \omega_{p}^{2}+\omega_{k}^{6} \\
& \left.-3 \omega_{j}^{4} \omega_{p}^{2}+10 \omega_{i}^{2} \omega_{k}^{2} \omega_{j}^{2}+\omega_{j}^{6}-2 \omega_{k}^{2} \omega_{j}^{2} \omega_{p}^{2}-3 \omega_{i}^{4} \omega_{p}^{2}-\omega_{j}^{2} \omega_{i}^{4}\right)\left(h_{i j k}^{p}+A_{i j k}^{p}+A_{k i j}^{p}+A_{j i k}^{p}\right) \\
& +\left(4 \omega_{i}^{2} \omega_{k}^{2} \omega_{j}^{4}-4 \omega_{k}^{2} \omega_{j}^{4} \omega_{p}^{2}+4 \omega_{i}^{2} \omega_{k}^{4} \omega_{j}^{2}-4 \omega_{j}^{2} \omega_{k}^{4} \omega_{p}^{2}-6 \omega_{i}^{4} \omega_{k}^{2} \omega_{j}^{2}+2 \omega_{k}^{2} \omega_{j}^{2} \omega_{p}^{4}\right. \\
& \left.+4 \omega_{i}^{2} \omega_{k}^{2} \omega_{j}^{2} \omega_{p}^{2}-4 \omega_{j}^{4} \omega_{k}^{4}+2 \omega_{k}^{2} \omega_{j}^{6}+2 \omega_{j}^{2} \omega_{k}^{6}\right) B_{i j k}^{p} \\
& +\left(4 \omega_{i}^{2} \omega_{k}^{4} \omega_{j}^{2}+4 \omega_{i}^{2} \omega_{k}^{2} \omega_{j}^{2} \omega_{p}^{2}+2 \omega_{i}^{2} \omega_{k}^{2} \omega_{p}^{4}+2 \omega_{i}^{2} \omega_{k}^{6}+2 \omega_{i}^{6} \omega_{k}^{2}-4 \omega_{i}^{4} \omega_{k}^{4}\right. \\
& \left.-6 \omega_{i}^{2} \omega_{k}^{2} \omega_{j}^{4}-4 \omega_{i}^{2} \omega_{k}^{4} \omega_{p}^{2}-4 \omega_{i}^{4} \omega_{k}^{2} \omega_{p}^{2}+4 \omega_{i}^{4} \omega_{k}^{2} \omega_{j}^{2}\right) B_{j i k}^{p} \\
& +\left(\quad-4 \omega_{i}^{4} \omega_{j}^{4}+2 \omega_{i}^{2} \omega_{j}^{2} \omega_{p}^{4}-6 \omega_{i}^{2} \omega_{k}^{4} \omega_{j}^{2}+2 \omega_{i}^{2} \omega_{j}^{6}+2 \omega_{i}^{6} \omega_{j}^{2}-4 \omega_{i}^{2} \omega_{j}^{4} \omega_{p}^{2}\right. \\
& \left.\left.+4 \omega_{i}^{2} \omega_{k}^{2} \omega_{j}^{2} \omega_{p}^{2}+4 \omega_{i}^{2} \omega_{k}^{2} \omega_{j}^{4}-4 \omega_{i}^{4} \omega_{j}^{2} \omega_{p}^{2}+4 \omega_{i}^{4} \omega_{k}^{2} \omega_{j}^{2}\right) B_{k i j}^{p}\right] \\
& \mu_{i j k}^{p}=\frac{1}{D_{i j k p}^{(3)}}\left[\quad \quad-4 \omega_{i}^{2} \omega_{j}^{2}+4 \omega_{i}^{2} \omega_{p}^{2}+12 \omega_{i}^{2} \omega_{k}^{2}-4 \omega_{k}^{2} \omega_{j}^{2}+4 \omega_{k}^{2} \omega_{p}^{2}+2 \omega_{p}^{4}\right. \\
& \left.+10 \omega_{j}^{4}-6 \omega_{i}^{4}-6 \omega_{k}^{4}-12 \omega_{j}^{2} \omega_{p}^{2}\right)\left(h_{i j k}^{p}+A_{i j k}^{p}+A_{k i j}^{p}+A_{j i k}^{p}\right) \\
& +\left(10 \omega_{i}^{2} \omega_{k}^{2} \omega_{j}^{2}-2 \omega_{k}^{2} \omega_{j}^{2} \omega_{p}^{2}+2 \omega_{i}^{2} \omega_{k}^{2} \omega_{p}^{2}-6 \omega_{j}^{2} \omega_{i}^{2} \omega_{p}^{2}+9 \omega_{k}^{2} \omega_{j}^{4}\right. \\
& +3 \omega_{j}^{6}-11 \omega_{k}^{4} \omega_{j}^{2}+\omega_{k}^{2} \omega_{p}^{4}+3 \omega_{i}^{2} \omega_{k}^{4}+\omega_{k}^{4} \omega_{p}^{2}-\omega_{k}^{6}-3 \omega_{i}^{4} \omega_{p}^{2} \\
& \left.+3 \omega_{i}^{2} \omega_{p}^{4}+\omega_{i}^{6}-\omega_{p}^{6}+5 \omega_{j}^{2} \omega_{p}^{4}-7 \omega_{j}^{4} \omega_{p}^{2}-5 \omega_{j}^{4} \omega_{i}^{2}+\omega_{j}^{2} \omega_{i}^{4}-3 \omega_{i}^{4} \omega_{k}^{2}\right) B_{i j k}^{p} \\
& +\left(-18 \omega_{i}^{2} \omega_{k}^{2} \omega_{j}^{2}-3 \omega_{j}^{2} \omega_{p}^{4}+5 \omega_{k}^{4} \omega_{j}^{2}+5 \omega_{j}^{2} \omega_{i}^{4}-\omega_{k}^{2} \omega_{j}^{4}-\omega_{j}^{4} \omega_{i}^{2}+7 \omega_{i}^{4} \omega_{p}^{2}\right. \\
& +3 \omega_{i}^{2} \omega_{k}^{4}+\omega_{p}^{6}-\omega_{j}^{6}-3 \omega_{k}^{6}-3 \omega_{i}^{6}+3 \omega_{j}^{4} \omega_{p}^{2}+7 \omega_{k}^{4} \omega_{p}^{2}+3 \omega_{i}^{4} \omega_{k}^{2}+6 \omega_{k}^{2} \omega_{j}^{2} \omega_{p}^{2} \\
& \left.+6 \omega_{j}^{2} \omega_{i}^{2} \omega_{p}^{2}-5 \omega_{k}^{2} \omega_{p}^{4}-6 \omega_{i}^{2} \omega_{k}^{2} \omega_{p}^{2}-5 \omega_{i}^{2} \omega_{p}^{4}\right) B_{j i k}^{p} \\
& +\left(\quad \omega_{k}^{4} \omega_{j}^{2}+\omega_{k}^{6}-2 \omega_{j}^{2} \omega_{i}^{2} \omega_{p}^{2}+10 \omega_{i}^{2} \omega_{k}^{2} \omega_{j}^{2}+3 \omega_{k}^{2} \omega_{p}^{4}-6 \omega_{k}^{2} \omega_{j}^{2} \omega_{p}^{2}-3 \omega_{i}^{2} \omega_{k}^{4}\right. \\
& +2 \omega_{i}^{2} \omega_{k}^{2} \omega_{p}^{2}+5 \omega_{j}^{2} \omega_{p}^{4}+3 \omega_{i}^{4} \omega_{k}^{2}-11 \omega_{j}^{2} \omega_{i}^{4}-3 \omega_{k}^{4} \omega_{p}^{2}+\omega_{i}^{4} \omega_{p}^{2}+3 \omega_{j}^{6} \\
& \left.\left.+\omega_{i}^{2} \omega_{p}^{4}-\omega_{i}^{6}-\omega_{p}^{6}+9 \omega_{j}^{4} \omega_{i}^{2}-7 \omega_{j}^{4} \omega_{p}^{2}-5 \omega_{k}^{2} \omega_{j}^{4}\right) B_{k i j}^{p}\right]
\end{aligned}
$$




$$
\begin{aligned}
& \nu_{k i j}^{p}=\frac{1}{D_{i j k p}^{(3)}}\left[\quad-2 \omega_{j}^{2} \omega_{i}^{2} \omega_{p}^{2}+\omega_{j}^{4} \omega_{p}^{2}-\omega_{j}^{6}+3 \omega_{k}^{2} \omega_{j}^{4}+\omega_{k}^{6}-7 \omega_{i}^{4} \omega_{p}^{2}-11 \omega_{j}^{4} \omega_{i}^{2}+2 \omega_{k}^{2} \omega_{j}^{2} \omega_{p}^{2}\right. \\
& +9 \omega_{j}^{2} \omega_{i}^{4}+3 \omega_{i}^{6}+5 \omega_{i}^{2} \omega_{p}^{4}+\omega_{j}^{2} \omega_{p}^{4}-5 \omega_{i}^{4} \omega_{k}^{2}+10 \omega_{i}^{2} \omega_{k}^{2} \omega_{j}^{2}-6 \omega_{i}^{2} \omega_{k}^{2} \omega_{p}^{2}+3 \omega_{k}^{2} \omega_{p}^{4} \\
& \left.-3 \omega_{k}^{4} \omega_{p}^{2}-\omega_{p}^{6}+\omega_{i}^{2} \omega_{k}^{4}-3 \omega_{k}^{4} \omega_{j}^{2}\right)\left(h_{i j k}^{p}+A_{i j k}^{p}+A_{k i j}^{p}+A_{j i k}^{p}\right) \\
& +\left(3 \omega_{i}^{4} \omega_{p}^{4}+\omega_{i}^{8}-6 \omega_{i}^{4} \omega_{k}^{2} \omega_{p}^{2}-5 \omega_{i}^{4} \omega_{k}^{4}+6 \omega_{i}^{2} \omega_{k}^{2} \omega_{j}^{2} \omega_{p}^{2}+5 \omega_{i}^{2} \omega_{j}^{2} \omega_{p}^{4}-7 \omega_{i}^{2} \omega_{j}^{4} \omega_{p}^{2}\right. \\
& +18 \omega_{i}^{4} \omega_{k}^{2} \omega_{j}^{2}+3 \omega_{i}^{2} \omega_{k}^{6}-3 \omega_{i}^{2} \omega_{k}^{2} \omega_{j}^{4}-7 \omega_{i}^{2} \omega_{k}^{4} \omega_{p}^{2}-6 \omega_{i}^{4} \omega_{j}^{2} \omega_{p}^{2}+\omega_{i}^{6} \omega_{k}^{2}-3 \omega_{i}^{2} \omega_{k}^{4} \omega_{j}^{2} \\
& \left.+5 \omega_{i}^{2} \omega_{k}^{2} \omega_{p}^{4}+\omega_{i}^{6} \omega_{j}^{2}-5 \omega_{i}^{4} \omega_{j}^{4}-3 \omega_{i}^{6} \omega_{p}^{2}-\omega_{i}^{2} \omega_{p}^{6}+3 \omega_{i}^{2} \omega_{j}^{6}\right) B_{j i k}^{p} \\
& +\left(3 \omega_{k}^{2} \omega_{j}^{6}+6 \omega_{i}^{2} \omega_{j}^{4} \omega_{p}^{2}+5 \omega_{i}^{4} \omega_{j}^{4}+7 \omega_{i}^{4} \omega_{j}^{2} \omega_{p}^{2}-9 \omega_{i}^{4} \omega_{k}^{2} \omega_{j}^{2}-\omega_{i}^{2} \omega_{j}^{6}+\omega_{p}^{6} \omega_{j}^{2}-\omega_{j}^{2} \omega_{k}^{4} \omega_{p}^{2}\right. \\
& -3 \omega_{p}^{4} \omega_{j}^{4}+3 \omega_{j}^{6} \omega_{p}^{2}+11 \omega_{i}^{2} \omega_{k}^{4} \omega_{j}^{2}-3 \omega_{i}^{6} \omega_{j}^{2}-5 \omega_{i}^{2} \omega_{j}^{2} \omega_{p}^{4}-2 \omega_{k}^{2} \omega_{j}^{4} \omega_{p}^{2}-\omega_{k}^{2} \omega_{j}^{2} \omega_{p}^{4} \\
& \left.+2 \omega_{i}^{2} \omega_{k}^{2} \omega_{j}^{2} \omega_{p}^{2}+\omega_{j}^{2} \omega_{k}^{6}-3 \omega_{j}^{4} \omega_{k}^{4}-10 \omega_{i}^{2} \omega_{k}^{2} \omega_{j}^{4}-\omega_{j}^{8}\right) B_{i j k}^{p} \\
& +\left(-4 \omega_{i}^{4} \omega_{k}^{2} \omega_{j}^{2}-6 \omega_{i}^{2} \omega_{k}^{4} \omega_{j}^{2}-4 \omega_{i}^{4} \omega_{j}^{4}-12 \omega_{i}^{4} \omega_{j}^{2} \omega_{p}^{2}+2 \omega_{i}^{2} \omega_{j}^{2} \omega_{p}^{4}+4 \omega_{i}^{2} \omega_{j}^{4} \omega_{p}^{2}\right. \\
& \left.\left.+12 \omega_{i}^{2} \omega_{k}^{2} \omega_{j}^{4}+10 \omega_{i}^{6} \omega_{j}^{2}-6 \omega_{i}^{2} \omega_{j}^{6}+4 \omega_{i}^{2} \omega_{k}^{2} \omega_{j}^{2} \omega_{p}^{2}\right) B_{k i j}^{p}\right] \\
& \nu_{j i k}^{p}=\frac{1}{D_{i j k p}^{(3)}}\left[\quad \left(\quad \omega_{i}^{2} \omega_{k}^{4}+\omega_{i}^{4} \omega_{p}^{2}+3 \omega_{j}^{2} \omega_{i}^{4}+\omega_{k}^{4} \omega_{p}^{2}-\omega_{p}^{6}-3 \omega_{j}^{4} \omega_{p}^{2}+3 \omega_{j}^{2} \omega_{p}^{4}+2 \omega_{j}^{2} \omega_{i}^{2} \omega_{p}^{2}+\omega_{j}^{6}\right.\right. \\
& +3 \omega_{k}^{4} \omega_{j}^{2}+\omega_{i}^{2} \omega_{p}^{4}+2 \omega_{k}^{2} \omega_{j}^{2} \omega_{p}^{2}-\omega_{k}^{6}+2 \omega_{i}^{2} \omega_{k}^{2} \omega_{j}^{2}-10 \omega_{i}^{2} \omega_{k}^{2} \omega_{p}^{2}-3 \omega_{j}^{4} \omega_{i}^{2} \\
& \left.+\omega_{i}^{4} \omega_{k}^{2}-3 \omega_{k}^{2} \omega_{j}^{4}-\omega_{i}^{6}+\omega_{k}^{2} \omega_{p}^{4}\right)\left(h_{i j k}^{p}+A_{i j k}^{p}+A_{k i j}^{p}+A_{j i k}^{p}\right) \\
& +\left(-3 \omega_{i}^{4} \omega_{k}^{4}-\omega_{i}^{2} \omega_{k}^{2} \omega_{p}^{4}-2 \omega_{i}^{2} \omega_{k}^{4} \omega_{j}^{2}-2 \omega_{i}^{2} \omega_{k}^{4} \omega_{p}^{2}-\omega_{i}^{4} \omega_{k}^{2} \omega_{p}^{2}+\omega_{i}^{6} \omega_{k}^{2}\right. \\
& +\omega_{k}^{2} \omega_{j}^{6}-2 \omega_{j}^{2} \omega_{k}^{4} \omega_{p}^{2}-\omega_{i}^{4} \omega_{k}^{2} \omega_{j}^{2}+3 \omega_{i}^{2} \omega_{k}^{6}-\omega_{k}^{2} \omega_{j}^{2} \omega_{p}^{4}-3 \omega_{p}^{4} \omega_{k}^{4}+3 \omega_{k}^{6} \omega_{p}^{2}-\omega_{k}^{2} \omega_{j}^{4} \omega_{p}^{2} \\
& \left.-\omega_{k}^{8}+10 \omega_{i}^{2} \omega_{k}^{2} \omega_{j}^{2} \omega_{p}^{2}+3 \omega_{j}^{2} \omega_{k}^{6}-3 \omega_{j}^{4} \omega_{k}^{4}-\omega_{i}^{2} \omega_{k}^{2} \omega_{j}^{4}+\omega_{k}^{2} \omega_{p}^{6}\right) B_{i j k}^{p} \\
& +\left(-4 \omega_{i}^{2} \omega_{k}^{2} \omega_{j}^{2} \omega_{p}^{2}+4 \omega_{i}^{2} \omega_{k}^{4} \omega_{j}^{2}+4 \omega_{i}^{4} \omega_{k}^{2} \omega_{j}^{2}-2 \omega_{i}^{2} \omega_{k}^{6}+6 \omega_{i}^{2} \omega_{k}^{2} \omega_{p}^{4}-4 \omega_{i}^{4} \omega_{k}^{2} \omega_{p}^{2}\right. \\
& \left.-2 \omega_{i}^{6} \omega_{k}^{2}+4 \omega_{i}^{4} \omega_{k}^{4}-2 \omega_{i}^{2} \omega_{k}^{2} \omega_{j}^{4}-4 \omega_{i}^{2} \omega_{k}^{4} \omega_{p}^{2}\right) B_{j i k}^{p} \\
& +\left(\quad-2 \omega_{i}^{4} \omega_{j}^{2} \omega_{p}^{2}+3 \omega_{i}^{6} \omega_{k}^{2}-3 \omega_{i}^{4} \omega_{k}^{4}+3 \omega_{i}^{6} \omega_{p}^{2}-3 \omega_{i}^{4} \omega_{p}^{4}-\omega_{i}^{2} \omega_{j}^{2} \omega_{p}^{4}-2 \omega_{i}^{4} \omega_{k}^{2} \omega_{p}^{2}\right. \\
& -\omega_{i}^{8}-\omega_{i}^{2} \omega_{k}^{4} \omega_{p}^{2}+\omega_{i}^{2} \omega_{j}^{6}-2 \omega_{i}^{4} \omega_{k}^{2} \omega_{j}^{2}-\omega_{i}^{2} \omega_{k}^{2} \omega_{p}^{4}+3 \omega_{i}^{6} \omega_{j}^{2}+10 \omega_{i}^{2} \omega_{k}^{2} \omega_{j}^{2} \omega_{p}^{2} \\
& \left.\left.+\omega_{i}^{2} \omega_{k}^{6}+\omega_{i}^{2} \omega_{p}^{6}-\omega_{i}^{2} \omega_{k}^{2} \omega_{j}^{4}-3 \omega_{i}^{4} \omega_{j}^{4}-\omega_{i}^{2} \omega_{j}^{4} \omega_{p}^{2}-\omega_{i}^{2} \omega_{k}^{4} \omega_{j}^{2}\right) B_{k i j}^{p}\right] \\
& \nu_{i j k}^{p}=\frac{1}{D_{i j k p}^{(3)}}\left[\quad \left(\quad-11 \omega_{k}^{2} \omega_{j}^{4}-3 \omega_{i}^{4} \omega_{p}^{2}+9 \omega_{k}^{4} \omega_{j}^{2}-\omega_{p}^{6}+\omega_{j}^{4} \omega_{p}^{2}-7 \omega_{k}^{4} \omega_{p}^{2}-\omega_{j}^{6}+\omega_{i}^{4} \omega_{k}^{2}\right.\right. \\
& +2 \omega_{j}^{2} \omega_{i}^{2} \omega_{p}^{2}-3 \omega_{j}^{2} \omega_{i}^{4}+3 \omega_{k}^{6}-6 \omega_{i}^{2} \omega_{k}^{2} \omega_{p}^{2}+\omega_{j}^{2} \omega_{p}^{4}+3 \omega_{i}^{2} \omega_{p}^{4}+5 \omega_{k}^{2} \omega_{p}^{4}-5 \omega_{i}^{2} \omega_{k}^{4} \\
& \left.+\omega_{i}^{6}+3 \omega_{j}^{4} \omega_{i}^{2}-2 \omega_{k}^{2} \omega_{j}^{2} \omega_{p}^{2}+10 \omega_{i}^{2} \omega_{k}^{2} \omega_{j}^{2}\right)\left(h_{i j k}^{p}+A_{i j k}^{p}+A_{k i j}^{p}+A_{j i k}^{p}\right) \\
& +\left(12 \omega_{i}^{2} \omega_{k}^{2} \omega_{j}^{4}-6 \omega_{k}^{2} \omega_{j}^{6}-4 \omega_{i}^{2} \omega_{k}^{4} \omega_{j}^{2}+4 \omega_{i}^{2} \omega_{k}^{2} \omega_{j}^{2} \omega_{p}^{2}+4 \omega_{k}^{2} \omega_{j}^{4} \omega_{p}^{2}\right. \\
& \left.+2 \omega_{k}^{2} \omega_{j}^{2} \omega_{p}^{4}-4 \omega_{j}^{4} \omega_{k}^{4}-12 \omega_{j}^{2} \omega_{k}^{4} \omega_{p}^{2}-6 \omega_{i}^{4} \omega_{k}^{2} \omega_{j}^{2}+10 \omega_{j}^{2} \omega_{k}^{6}\right) B_{i j k}^{p} \\
& +\left(3 \omega_{k}^{2} \omega_{j}^{6}+6 \omega_{i}^{2} \omega_{k}^{2} \omega_{j}^{2} \omega_{p}^{2}+\omega_{j}^{2} \omega_{k}^{6}-3 \omega_{i}^{2} \omega_{k}^{2} \omega_{j}^{4}-\omega_{k}^{2} \omega_{p}^{6}-3 \omega_{i}^{4} \omega_{k}^{2} \omega_{j}^{2}\right. \\
& +18 \omega_{i}^{2} \omega_{k}^{4} \omega_{j}^{2}-6 \omega_{i}^{2} \omega_{k}^{4} \omega_{p}^{2}-5 \omega_{j}^{4} \omega_{k}^{4}+3 \omega_{i}^{6} \omega_{k}^{2}+\omega_{i}^{2} \omega_{k}^{6}+5 \omega_{i}^{2} \omega_{k}^{2} \omega_{p}^{4}-6 \omega_{j}^{2} \omega_{k}^{4} \omega_{p}^{2} \\
& \left.+\omega_{k}^{8}+5 \omega_{k}^{2} \omega_{j}^{2} \omega_{p}^{4}-3 \omega_{k}^{6} \omega_{p}^{2}+3 \omega_{p}^{4} \omega_{k}^{4}-7 \omega_{i}^{4} \omega_{k}^{2} \omega_{p}^{2}-5 \omega_{i}^{4} \omega_{k}^{4}-7 \omega_{k}^{2} \omega_{j}^{4} \omega_{p}^{2}\right) B_{j i k}^{p} \\
& +\left(-\omega_{i}^{4} \omega_{j}^{2} \omega_{p}^{2}-\omega_{j}^{8}-5 \omega_{k}^{2} \omega_{j}^{2} \omega_{p}^{4}+7 \omega_{j}^{2} \omega_{k}^{4} \omega_{p}^{2}+5 \omega_{j}^{4} \omega_{k}^{4}+3 \omega_{j}^{6} \omega_{p}^{2}-\omega_{k}^{2} \omega_{j}^{6}\right. \\
& -3 \omega_{p}^{4} \omega_{j}^{4}+\omega_{i}^{6} \omega_{j}^{2}+2 \omega_{i}^{2} \omega_{k}^{2} \omega_{j}^{2} \omega_{p}^{2}+11 \omega_{i}^{4} \omega_{k}^{2} \omega_{j}^{2}+\omega_{p}^{6} \omega_{j}^{2}-10 \omega_{i}^{2} \omega_{k}^{2} \omega_{j}^{4}-3 \omega_{j}^{2} \omega_{k}^{6} \\
& \left.\left.-3 \omega_{i}^{4} \omega_{j}^{4}-9 \omega_{i}^{2} \omega_{k}^{4} \omega_{j}^{2}+3 \omega_{i}^{2} \omega_{j}^{6}+6 \omega_{k}^{2} \omega_{j}^{4} \omega_{p}^{2}-\omega_{i}^{2} \omega_{j}^{2} \omega_{p}^{4}-2 \omega_{i}^{2} \omega_{j}^{4} \omega_{p}^{2}\right) B_{k i j}^{p}\right]
\end{aligned}
$$

where $D_{i j k p}^{(3)}=\left(\omega_{k}+\omega_{i}-\omega_{p}-\omega_{j}\right)\left(\omega_{k}+\omega_{i}-\omega_{p}+\omega_{j}\right)\left(-\omega_{k}+\omega_{i}+\omega_{p}+\omega_{j}\right)\left(-\omega_{k}+\omega_{i}+\omega_{p}-\right.$ $\left.\omega_{j}\right)\left(\omega_{k}+\omega_{i}+\omega_{p}-\omega_{j}\right)\left(\omega_{k}+\omega_{i}+\omega_{p}+\omega_{j}\right)\left(-\omega_{k}+\omega_{i}-\omega_{p}+\omega_{j}\right)\left(-\omega_{k}+\omega_{i}-\omega_{p}-\omega_{j}\right)$. 